San Jose State University

SJSU ScholarWorks

Master's Theses

Master's Theses and Graduate Research

1999

\title{
Synthesis and characterization of a new chemically bonded liquid crystal stationary phase for HPLC
}

Sheela Muley

San Jose State University

Follow this and additional works at: https://scholarworks.sjsu.edu/etd_theses

\section{Recommended Citation}

Muley, Sheela, "Synthesis and characterization of a new chemically bonded liquid crystal stationary phase for HPLC" (1999). Master's Theses. 1890.

DOI: https://doi.org/10.31979/etd.u58s-mxnz

https://scholarworks.sjsu.edu/etd_theses/1890

This Thesis is brought to you for free and open access by the Master's Theses and Graduate Research at SJSU ScholarWorks. It has been accepted for inclusion in Master's Theses by an authorized administrator of SJSU ScholarWorks. For more information, please contact scholarworks@sjsu.edu. 


\section{INFORMATION TO USERS}

This manuscript has been reproduced from the microfilm master. UMI films the text directly from the original or copy submitted. Thus, some thesis and dissertation copies are in typewriter face, while others may be from any type of computer printer.

The quality of this reproduction is dependent upon the quality of the copy submitted. Broken or indistinct print, colored or poor quality illustrations and photographs, print bleedthrough, substandard margins, and improper alignment can adversely affect reproduction.

In the unlikely event that the author did not send UMI a complete manuscript and there are missing pages, these will be noted. Also, if unauthorized copyright material had to be removed, a note will indicate the deletion.

Oversize materials (e.g., maps, drawings, charts) are reproduced by sectioning the original, beginning at the upper left-hand comer and continuing from left to right in equal sections with small overlaps. Each original is also photographed in one exposure and is included in reduced form at the back of the book.

Photographs included in the original manuscript have been reproduced xerographically in this copy. Higher quality $6^{n} \times 9^{\prime \prime}$ black and white photographic prints are available for any photographs or illustrations appearing in this copy for an additional charge. Contact UMI directly to order.

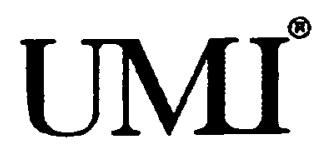

Bell \& Howell Information and Leaming 300 North Zeeb Road, Ann Arbor, MI 48106-1346 USA $800-521-0600$ 

BONDED LIQUID CRYSTAL STATIONARY PHASE FOR HPLC

\author{
A Thesis \\ Presented to \\ The Faculty of The Department of Chemistry \\ San Jose State University
}

\author{
In Partial Fulfillment \\ of the Requirement for the Degree \\ Master of Science
}

By

Sheela Muley

August, 1999 
UMI Number: 1396191

UMI Microform 1396191

Copyright 1999, by UMI Company. All rights reserved.

This microform edition is protected against unauthorized copying under Title 17, United States Code.

\author{
UMI \\ 300 North Zeeb Road \\ Ann Arbor, MI 48103
}


(C) 1999

Sheela Muley

ALL RIGHTS RESERVED 
APPROVED FOR THE DEPARTMENT OF CHEMISTRY
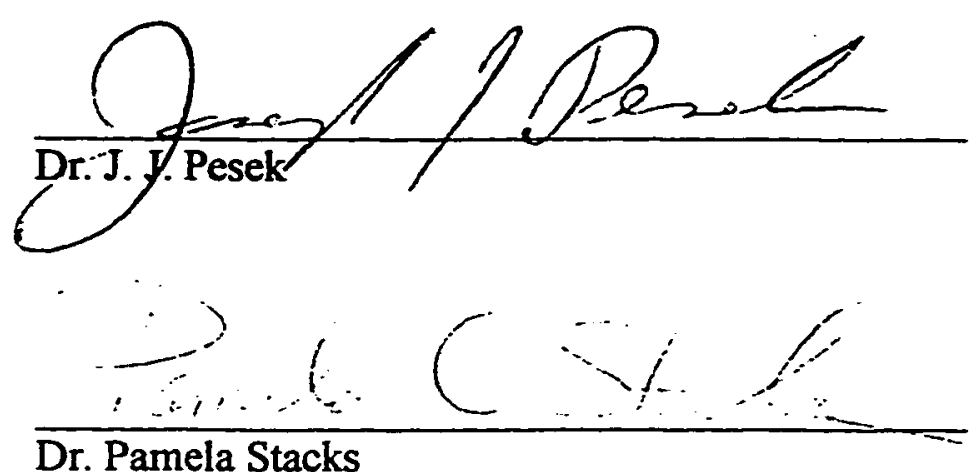

Dr. Pamela Stacks

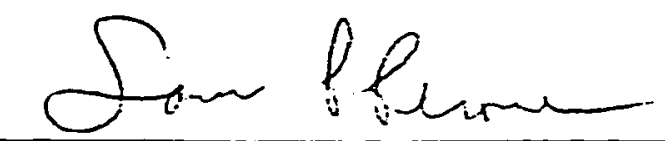

Dr. Sam Prone

APPROVED FOR THE UNIVERSITY

William Fish 


\section{ABSTRACT \\ SYNTHESIS AND CHARACTERIZATION OF A NEW CHEMICALLY BONDED \\ LIQUID CRYSTAL STATIONARY PHASE FOR HPLC}

by

Sheela Muley

Liquid crystals or 'mesophases' are a state of matter that is intermediate between a solid crystalline phase and the liquid phase. The goal of this research was to synthesize a novel chemically bonded liquid crystal stationary phase for HPLC. In this study two chemically bonded liquid crystal stationary phases were synthesized and their spectroscopic and chromatographic properties were characterized. The liquid crystal materials were bonded to silica via a free radical initiation reaction. The procedure involved preparation of a stable silica hydride intermediate (silanization) followed by free radical initiation to obtain the bonded phase.

Diffuse reflectance infrared Fourier transform (DRIFT) spectroscopy and crosspolarization magic-angle spinning nuclear magnetic resonance (CP-MAS NMR) spectroscopy were used for the characterization of these bonded phases. The bonded phases were also evaluated chromatographically by testing the retention and selectivity of benzodiazipines and polycyclic aromatic hydrocarbons (PAH's). A comparative study was then devised based on the spectroscopic and chromatographic results. 


\section{ACKNOWLEDGMENTS}

I would like to thank my advisor, Dr. Joseph Pesek and his project scientist Dr. Maria Matyska-Pesek for giving me this opportunity to work with them on this project. I appreciate their patience and encouragement during my entire work on this project. I would also like to thank my committee members, Dr. Pamela Stacks and Dr. Sam Perone for reviewing my thesis. I would like to thank my in-laws and my husband for ail the help and support. This project could not have been completed without their support and encouragement. Finally, I would like to thank my nine months old son, Dhruv, for being such a good baby and helping me finish this project.

Sheela Muley 


\section{TABLE OF CONTENTS}

\section{CHAPTER I -INTRODUCTION}

A. Silica

1. Modification of silica gel

2. Free Radical Initiation

B. High Performance Liquid Chromatography 6

1. Stationary phase

2. Mobile Phase

C. Liquid Crystals

1.Thermotropic liquid crystals

2. Lyotropic liquid crystals

3. Rod-like Liquid Crystal Phases

D. Liquid crystals in HPLC

E. Characterization methods

1. Fourier Transform Infrared Spectroscopy (FTIR)

2. Cross Polarization Magic angle Spinning Nuclear Magnetic Resonance Spectroscopy (CP-MAS NMR)

3. Elemental Analysis

F. Aims of this study

CHAPTER II - EXPERIMENTAL

A. Materials $\quad 22$

1. Chemicals 22

2.Chemicals for bonded phase synthesis 23

B. Bonded phase synthesis $\quad 24$

1. Synthesis of the silica hydride intermediate via the TES Silanization process 24

2. Preparation of Speier's catalyst 25

3. Kromasil silica hydride synthesis 26

4. Bonded phase synthesis $\quad 26$

5. Free radical hydrosilation $\quad 28$

6. Column Packing 29

C. Instrumentation $\quad 30$

1. Surface Characterization Techniques $\quad 30$

2. High Performance Liquid Chromatography 31

3. Elemental Analysis $\quad 32$ 
D. Surface Coverage

E. Hydrolytic Stability Test

CHAPTER III - RESULTS AND DISCUSSION

A. Elemental Analysis $\quad 34$

B. Characterization of Silica Hydride $\quad 35$

1.DRIFT spectra $\quad 35$

$2{ }^{29} \mathrm{Si}$ CP-MAS NMR spectra

C. Cholesteryl 10-undecanoate bonded phase characterization 39

1. DRIFT spectrum $\quad 39$

2. ${ }^{13} \mathrm{C}$ CP-MAS NMR of unbonded cholestereyl 10 -undecanoate 40

3. ${ }^{13} \mathrm{C}$ CP-MAS NMR of bonded cholestereyl 10 -undecanoate 40

D. 1-(2-Cyanoethyl) pyrrole analysis $\quad 40$

1. DRIFT Spectra $\quad 40$

2. ${ }^{29} \mathrm{Si}$ CP-MAS NMR 46

3. ${ }^{13} \mathrm{C}$ CP-MAS NMR 46

4. Hydrolytic Stability Test $\quad 54$

E. 4-cyano-4'-n-pentyl-1,1'-biphenyl analysis $\quad 54$

1. DRIFT spectrum $\quad 54$

2. Hydrolytic Stability Test $\quad 58$

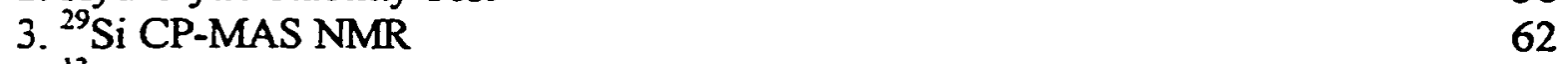

4. ${ }^{13} \mathrm{C}$ CP-MAS NMR $\quad 62$

F. 4-cyano-4'-n-pentoxy-1,1'-biphenyl analysis 65

1. DRIFT Spectra $\quad 65$

2. ${ }^{29} \mathrm{Si}$ CP-MAS NMR $\quad 67$

3a. ${ }^{13} \mathrm{C}$ CP-MAS NMR of starting material $\quad 72$

3b. ${ }^{13} \mathrm{C}$ CP-MAS NMR of bonded phase $\quad 72$

$\begin{array}{ll}\text { G. Chromatographic Studies } & \mathbf{7 4}\end{array}$

1. 4-cyano-4'- n- pentoxy-1,1'-biphenyl stationary phase 74

1. 4-Cyano-4'-n-pentyl-1,1'-biphenyl stationary phase 83

$\begin{array}{ll}\text { CHAPTER IV - CONCLUSION } & 92\end{array}$

CHAPTER V - REFERENCES 


\section{LIST OF FIGURES}

Figure 1. Reaction scheme for bonded phase synthesis 4

Figure 2. Cholesteryl 10-undecanoate bonded phase 5

Figure 3. Schematic representation of molecular order in different phases [12] 8

Figure 4. Schematic representation of nematic phase [12] 11

Figure 5. Schematic representation of the cholestric phase [12] 12

Figure 6. Schematic representation of the smectic A phase [12] 13

Figure 7. Schematic representation of the smectic $C$ phase [12] 13

Figure 8. Schematic representation of the "Slot model" [15] 14

Figure 9. Structure of 4-cyano-4'-n-pentyl-1,1'-biphenyl 16

Figure 10. Structure of 4-cyano-4'-n-pentoxy-1,1'-biphenyl 16

Figure 11. Reaction scheme for 4-cyano-4'-n-pentyl-1,1'-biphenyl bonded phase 17

Figure 12. Reaction scheme for 4-cyano-4'-n-pentoxy-1,1'-biphenyl bonded phase18

Figure 13. DRIFT spectrum of Kromasil silica hydride 35

Figure 14. DRIFT spectrum of Vydac silica hydride 36

Figure 15. The ${ }^{29}$ Si CP-MAS NMR spectrum of Kromasil silica hydride 37

Figure 16. The ${ }^{29}$ Si CP-MAS NMR spectrum of Vydac silica hydride 38

Figure 17. DRIFT spectrum of cholesteryl 10-undecanoate bonded to Vydac silica hydride

Figure 18. ${ }^{13} \mathrm{C}$ CP-MAS NMR spectrum of cholesteryl 10-undecanoate (before bonding) (taken from reference 25 )

Figure $19 .{ }^{13} \mathrm{C} \mathrm{CP-MAS} \mathrm{NMR} \mathrm{spectrum} \mathrm{of} \mathrm{cholesteryl} \mathrm{10-} \mathrm{undecanoate} \mathrm{bonded} \mathrm{to}$ Vydac silica hydride

Figure 20. DRIFT Spectrum of 1,2-Cyanoethyl pyrrole bonded to Kromasil silica hydride via Wilkinson's catalyst

Figure 21. DRIFT Spectrum of 1,2-Cyanoethyl pyrrole bonded to Kromasil hydride via AIBN

Figure 22. DRIFT Spectrum of 1,2-cyanoethyl pyrrole bonded to Kromasil hydride via $t$ - butyl peroxide.

Figure 23. ${ }^{29} \mathrm{Si} \mathrm{CP-MAS} \mathrm{NMR} \mathrm{spectrum} \mathrm{of} \mathrm{1,2-cyanoethyl} \mathrm{pyrrole} \mathrm{bonded} \mathrm{to}$ Kromasil hydride via Wilkinson's catalyst. 
Figure 24. ${ }^{29} \mathrm{Si}$ CP-MAS NMR spectrum of 1,2-cyanoethyl pyrrole bonded to Kromasil hydride via $t$-butyl peroxide

Figure $25 .{ }^{13} \mathrm{C}$ CP-MAS NMR spectrum of 1,2-cyanoethyl pyrrole bonded to

Kromasil hydride via AIBN

Figure 26. ${ }^{13} \mathrm{C}$ CP-MAS NMR spectrum of 1,2-cyanoethyl pyrrole bonded to

Kromasil hydride via $t$-butyl peroxide.

Figure $27 .{ }^{13} \mathrm{C}$ CP-MAS NMR spectrum of 1,2-cyanoethyl pyrrole bonded to

Kromasil hydride via Wilkinson's catalyst

Figure 28. DRIFT spectrum of 1,2 cyanoethyl pyrrole bonded to Kromasil hydride after acid hydrolysis

Figure 29. DRIFT spectrum of 1,2 cyanoethyl pyrrole bonded to Kromasil hydride after base hydrolysis

Figure 30. DRIFT spectrum of 4-cyano-4'-n-pentyl-1,1'-biphenyl bonded to

Kromasil silica hydride via free radical initiator tertiary butyl peroxide

Figure 31. DRIFT spectrum of 4-cyano-4'-n-pentyl-1,1'-biphenyl bonded to Vydac

silica hydride via free radical initiator tertiary butyl peroxide

Figure 32. DRIFT spectrum of 4-cyano-4"-n-pentyl-1, $1^{\circ}$-biphenyl bonded to

Kromasil hydride after acid hydrolysis

Figure 33. DRIFT spectrum of 4-cyano-4'-n-pentyl-1, $1^{-}$-biphenyl bonded to

Kromasil hydride after base hydrolysis.

Figure 34. ${ }^{29}$ Si CP-MAS NMR spectrum of 4-cyano-4 -n-pentyl-1,1'-biphenyl bonded to Kromasil hydride

Figure 35. ${ }^{13} \mathrm{C}$ CP-MAS NMR spectrum of 4-cyano-4'-n-pentyl-1, 1 -biphenyl bonded to Kromasil hydride at $50^{\circ} \mathrm{C}$

Figure 36. ${ }^{13} \mathrm{C}$ CP-MAS NMR spectrum of 4-cyano-4'-n-pentyl-1, $1^{\prime}$-biphenyl bonded to Kromasil hydride $\left(25^{\circ} \mathrm{C}\right)$

Figure 37. DRIFT spectrum of 4-cyano-4'-n-pentoxy-1,1'-biphenyl bonded to

Kromasil silica hydride via free radical initiator tertiary butyl peroxide

Figure 38. DRIFT spectrum of 4 - cyano-4'-n-pentoxy $-1,1$ '-bihenyl bonded to Vydac silica hydride via tertiary butyl peroxide 
Figure 39. ${ }^{29}$ Si CP-MAS NMR spectrum of 4-cyano-4'-n-pentoxy -1,1'-biphenyl bonded to Kromasil hydride

Figure 40. ${ }^{13} \mathrm{C}$ CP-MAS NMIR spectrum of 4-cyano-4'-n-pentoxy -1,1'-biphenyl starting material

Figure 41. ${ }^{13} \mathrm{C}$ CP-MAS NMR spectrum of 4-cyano-4'-n-pentoxy -1,1'-biphenyl bonded to Kromasil hydride

Figure 42. Reversed phase separation of SRM 869 mixture on a 4-cyano-4'-npentoxy-1,1'-biphenyl column; mobile phase : water : methanol, v/v (10: 90 ), at $0.5 \mathrm{~mL} / \mathrm{min}$; sample injection $20 \mu \mathrm{l}$; detection : UV at $254 \mathrm{~nm}$; peaks : $A=$ Benzo[a]pyrene, $\mathrm{B}=$ Phenanthro[3,4-c]phenanthrene and $\mathrm{C}=$ Tetrabenzonaphthalene

Figure 43. Reversed phase separation of PE universal test mixture on a 4-cyano-4'n-pentoxy-1,1'-biphenyl column; mobile phase : water : methanol, v/v (80: 20 ), at $0.5 \mathrm{ml} / \mathrm{min}$; sample injection $5 \mu \mathrm{l}$; detection : $\mathrm{UV}$ at $254 \mathrm{~nm}$; peaks : $A=$ benzene , $B=$ toulene,$C=$ ethylbenzene, $D=$ isopropyl benzene, $E$ : tertiary butyl benzene, anthracene was eluted at $50 \mathrm{~min}$.

Figure 44. Reversed phase separation of benzodiazipines mixture on a 4-cyano-4'-npentoxy-1,1'-biphenyl column; mobile phase : water : methanol, v/v ( 65 :35), at $0.4 \mathrm{~mL} / \mathrm{min}$; sample injection $20 \mu \mathrm{l}$; detection : UV at $254 \mathrm{~nm}$; peaks : $\mathrm{A}=$ Oxazepam + Clonazepam , B = Nitrazepam + Temazepam , C = Diazepam. 80

Figure 45. Reversed phase separation of benzodiazipines mixture on a 4-cyano-4'-npentoxy-1,1'-biphenyl column; mobile phase : water : methanol, v/v ( 75 :25 ), at $0.4 \mathrm{ml} / \mathrm{min}$; sample injection $20 \mu \mathrm{l}$; detection : $\mathrm{UV}$ at $254 \mathrm{~nm}$; peaks : $\mathrm{A}=$ Oxazepam + Clonazepam , B = Nitrazepam + Temazepam , C = Diazepam. 81

Figure 46. Reversed phase separation of NIST mixture (SRM 869) on a 4-cyano-4'n-pentyl-1, 1'-biphenyl column; mobile phase - water: methanol, v/v (30:70), at $0.5 \mathrm{~mL} / \mathrm{min}$; sample injection $20 \mu \mathrm{l}$; detection : UV at $254 \mathrm{~nm}$; peaks : $A=$ Benzo[a]pyrene, $\mathrm{B}+\mathrm{C}=$ Phenanthro[3,4-c]phenanthrene and Tetrabenzonaphthalene.

Figure 47. Reversed phase separation of NIST mixture (SRM 869) on a 4-cyano-4'n-pentyl-1,1'-biphenyl column; mobile phase : water : methanol, v/v ( 20 :80), 
at $0.5 \mathrm{~mL} / \mathrm{min}$; sample injection $20 \mu \mathrm{l}$; detection : $\mathrm{UV}$ at $254 \mathrm{~nm}$; peaks : $A=$ Benzo[a]pyrene, B + C = Phenanthro[3,4-c]phenanthrene and

Tetrabenzonaphthalene

Figure 48. Reversed phase separation of PE universal test mixture on a 4-cyano-4'n-pentyl-1,1'-biphenyl column; mobile phase : water : methanol, v/v (80 : 20 ), at $0.5 \mathrm{ml} / \mathrm{min}$; sample injection $20 \mu \mathrm{l}$; detection : $\mathrm{UV}$ at $254 \mathrm{~nm}$; peaks : peak A:benzene \& toluene; peak B: ethyl benzene, isopropyl benzene, $t$ - butyl benzene and anthracene

Figure 49. Reversed phase separation of benzodiazipines mixture on a 4-cyano-4'-npentyl-1,1'-biphenyl column; mobile phase : water : methanol, v/v (65:35), at $0.5 \mathrm{~mL} / \mathrm{min}$; sample injection $20 \mu \mathrm{l}$; detection : $\mathrm{UV}$ at $254 \mathrm{~nm}$; peaks : $A=$ Nitrazepam + Clonazepam + Oxazepam, B $=$ Temazepam + Diazepam

Figure 50. Reversed phase separation of benzodiazipines mixture on a 4-cyano-4'-npentyl-1,1'-biphenyl column; mobile phase : water : methanol, v/v (80: 20$)$, at $0.5 \mathrm{ml} / \mathrm{min}$; sample injection $20 \mu \mathrm{l}$; detection : $\mathrm{UV}$ at $254 \mathrm{~nm}$; peaks : $A=$ Solvent peak, $B=$ Nitrazepam + Clonazepam + Oxazepam, C $=$ Temazepam, D $=$ Diazepam 


\section{TABLE OF TABLES}

Table 1. Bonded Moiety

Table 2. CAS Registry numbers of the chemicals used

Table 3. Elemental Analysis

Table 4. Perkin-Elmer mixture (structures)

Table 5. Benzodiazipine structures

Table 6. Capacity factors of benzodiazipines on 4-Cyano-4'-N - Pentory-1,1'-Biphenyl column

Table 7. Capacity factor of benzodiazipine mixture using 4-Cyano-4'-N-Pentoxy-1,1'-Biphenyl column

Table 8. Capacity factors for benzodiazipine mixture using 4-Cyano-4'-N-Pentyl-1,1'-Biphenyl column

Table 9. Capacity factors for benzodiazipine mixture using 4-Cyano-4'-N-Pentyl-1,1'-Biphenyl column 


\section{CHAPTER I -INTRODUCTION}

\section{A. Silica}

$\mathrm{SiO}_{2}$ or silica gel is the most common solid support for HPLC. Other materials, such as titania, zirconia, graphite, polymers, etc., can also be used as the solid support in HPLC. However, silica is superior to all these materials in terms of rigidity, efficiency and performance. Silica gels used in chromatography are amorphous, non-crystalline materials, which do not produce any X-ray diffraction pattern. Amorphous silica is extremely porous in nature with a large internal surface area, which is responsible for greater analyte-stationary phase interactions. Moreover, silica has considerable mechanical strength and can be used under high pressure. It can be easily modified chemically by attaching an organic ligand. Chemical stability is responsible for solvent resistance. Silica is also available in a variety of pore and particle sizes. The surface chemistry of silica is important, as chromatographic processes, like adsoption take place on the surface. Porous silica contains silanols $(\mathrm{Si}-\mathrm{OH})$ on its surface. These silonols are considered as strong adsorption sites. It also contains exposed siloxane bonds ( $\mathrm{Si}-\mathrm{O}-\mathrm{Si}$ ) which are hydrophobic in nature. The siloxane bond is responsible for the retention of non-polar molecules due to its hydrophobic interaction with the solute [1]. The main drawback of using silica as the support material is its limited stability in aqueous solution outside of the $\mathrm{pH}$ range of 2 to 9 . Above $\mathrm{pH} 9$, the silica support is susceptible to dissolt:tion in aqueous solutions due to the formation of silicate ions. Below pH 2 , hydrolysis of the siloxane backbone can occur [2]. Chemically modifying the silica for its use as the stationary phase in HPLC has been practiced for more than 25 years. The modification of silica enhances the selectivity and versatility of the stationary phase [3]. 


\section{Modification of silica gel}

Since most stationary phases have silica as the support material, bonding of an organic ligand depends on the surface chemistry (silanols). There are several types of reactions that can be used for the attachment of an organic moiety to the silica surface. One type is an esterification which involves a reaction between an alcohol and the silanol. This reaction is called esterification because silica is the polymerization product of silicic acid.

$$
\equiv \mathrm{Si}-\mathrm{OH}+\mathrm{R}-\mathrm{OH} \rightarrow \mathrm{Si}-\mathrm{OR}+\mathrm{H}_{2} \mathrm{O}
$$

This reaction results in the formation of a Si-O-C linkage which is not very hydrolytically stable. Therefore this reaction is not suitable when dealing with aqueous mobile phases [3]. Another type is an organosilanization reaction between an organosilane reagent and the silanols.

i) $\quad . \mathrm{Si}-\mathrm{OH}+\mathrm{X}-\mathrm{SiR}_{2}{ }_{2} \mathrm{R} \rightarrow \mathrm{Si}-\mathrm{O}-\mathrm{SiR}_{2}{ }_{2} \mathrm{R}+\mathrm{HX}$

ii)

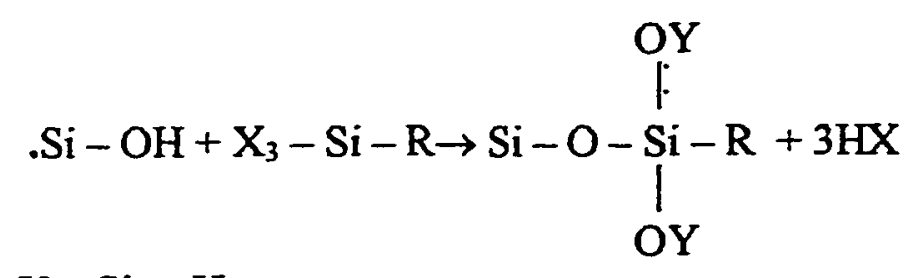

$\mathrm{Y}=\mathrm{Si}$ or $\mathrm{H}$

When the organosilane reagent has a single reactive group, it results in a monomeric stationary phase. A polymeric stationary phase is formed when there are three reactive groups on the organosilane reagent. This method results in the formation of a Si-O-Si-C linkage on the surface [3,4]. A third method involves chlorination of the silica surface with thionyl chloride first. This is followed by attachment of an organic compound through a Grignard reagent (BrMgR) or organolithium ( $\mathrm{Li}-\mathrm{R})$ compounds. This reaction results in a direct silicon-carbon linkage which is more hydrolytically stable [3,4]. 
i) $\mathrm{Si}-\mathrm{OH}+\mathrm{SOCl}_{2} \rightarrow \mathrm{Si}-\mathrm{Cl}+\mathrm{SO}_{2}+\mathrm{HCl}$

ii) $\mathrm{Si}-\mathrm{Cl}+\mathrm{BrMgR} \rightarrow \mathrm{Si}-\mathrm{R}+\mathrm{Mg} \mathrm{Cl} \mathrm{Br}$

$$
\mathrm{Si}-\mathrm{Cl}+\mathrm{Li}-\mathrm{R} \rightarrow \mathrm{Si}-\mathrm{R}+\mathrm{LiCl}
$$

However, this reaction requires extremely dry conditions and therefore it is not used frequently. Similar results can be achieved by an alternative method of silanization followed by a hydrosilation reaction $[3,4]$. This is a two-step process first introduced by Chu et al., [5]. In this method the silanols are first converted to hydrides which are then reacted with a terminal olefin in the presence of a suitable catalyst. The first reaction is called the silanization process which gives rise to a Si-H monolayer when using triethoxysilane (TES) as the reagent [6]. This step is followed by a hydrosilation process, involving a reaction between the silica hydride and a terminal olefin. This second step results in a Si-C linkage which follows anti-Markownikov addition of an olefin across a double bond [6]. Hydrosilation is carried out in the presence of hexachloroplatinic acid, commonly known as Speier's catalyst, first introduced by Speier to catalyze this reaction [7]. The complete process is shown in Figure 1.

The main advantage of the hydrosilation reaction is that a large number of functional groups can be bonded using a variety of catalysts. The silanization process also results in greater column efficiency due to a reduction in surface silanols [7]. A cholesteryl 10-undecanoate bonded phase was prepared using this hydrosilation process. The structure of the compound and the reaction product are shown below in Figure 2 . 
(1) TES Silanization

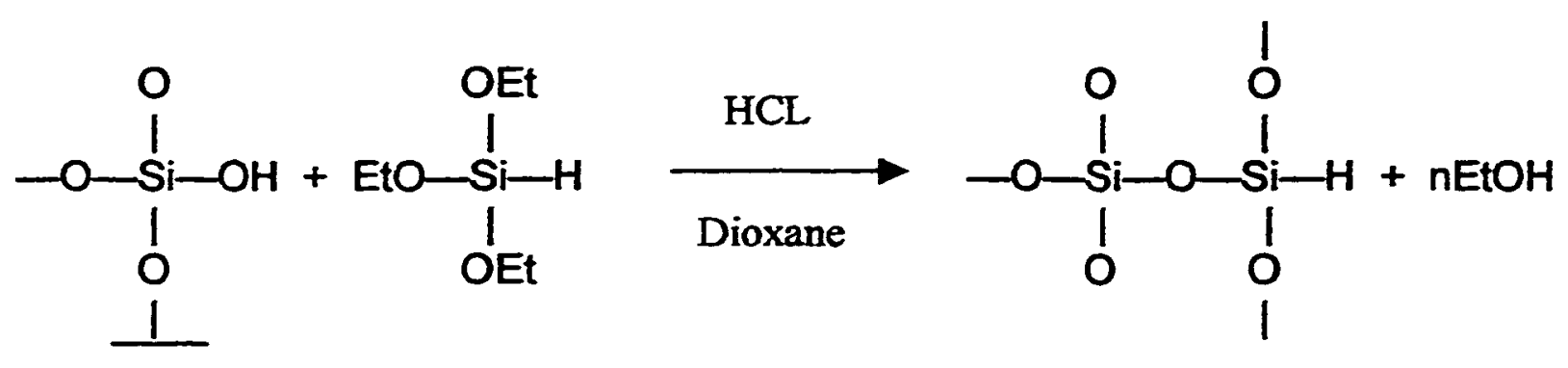

(2) Hydrosilation
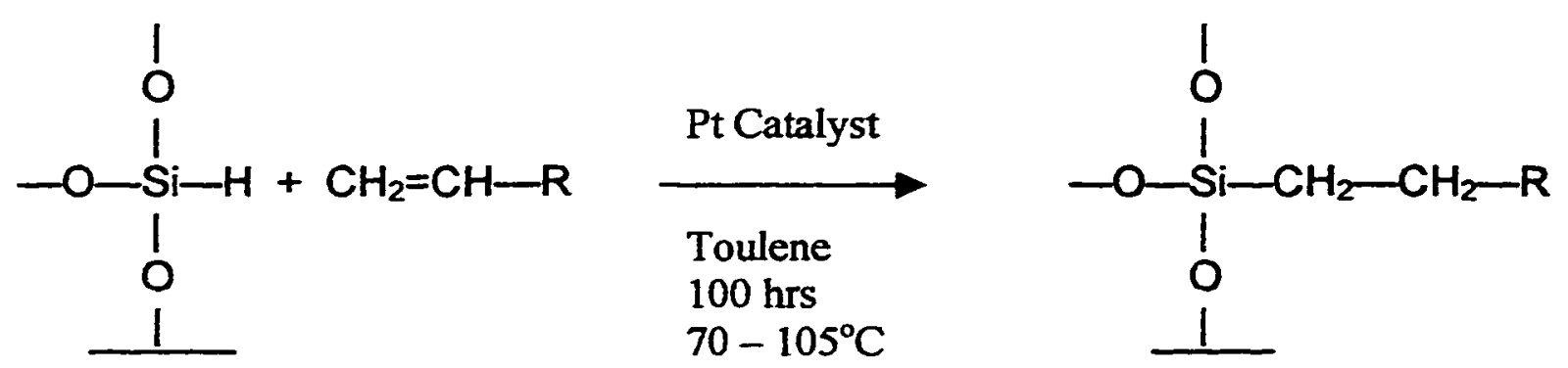

Figure 1. Reaction scheme for bonded phase synthesis

However, there are two problems associated with the hydrosilation process using a transition metal catalyst. The transition step in the bonding of the olefin to the hydride surface involves forming a complex with the catalyst. The intermediate species thus formed can be of significant size resulting in a lower yield of organic species on the surface. The other problem is the possible reduction of the catalyst itself. A reaction between the hydride and the metal would result in the deposition of $\mathrm{Pt}(0)$ on the surface. This might affect the chromatographic performance. Using free radical initiation instead of a transition metal complex for catalysis can eliminate these problems [8]. 


\section{Cholesteryl 10-undecenoate}

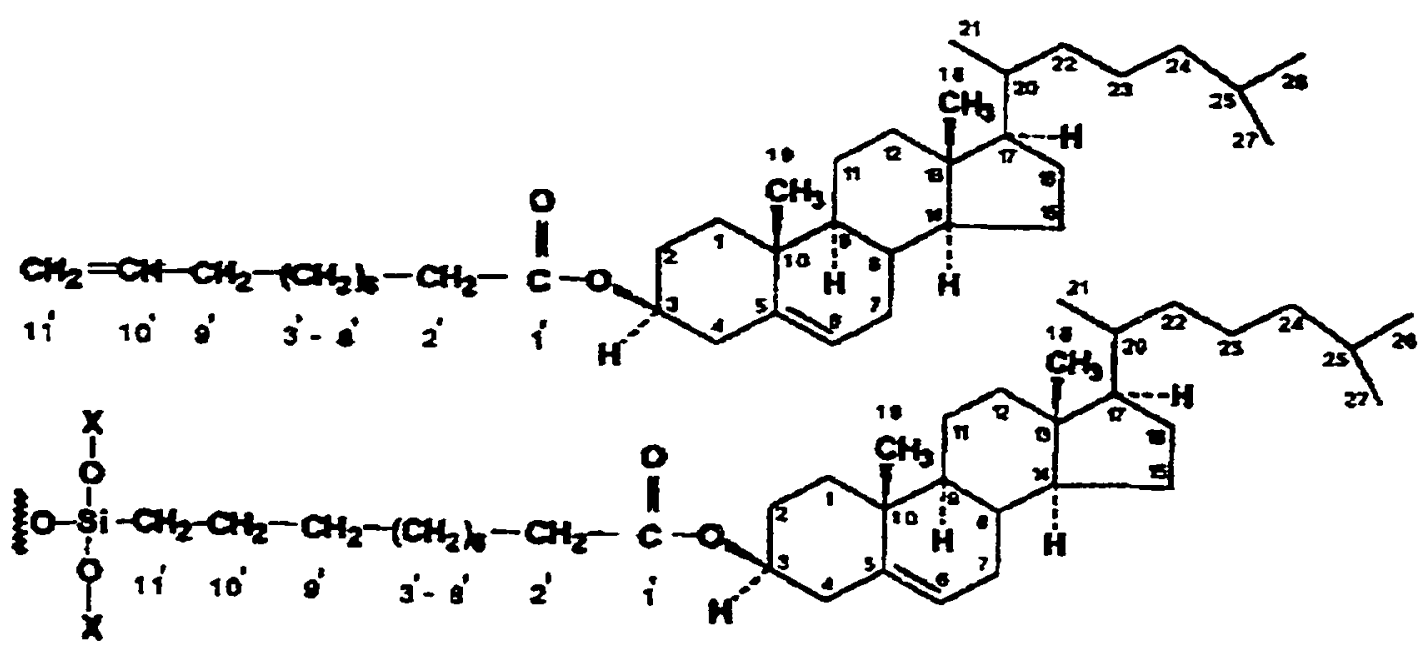

Figure 2. Cholesteryl 10-undecanoate bonded phase

2. Free Radical Initiation

In this study, two liquid crystal compounds each with a cyano moiety were used for the synthesis of stationary phases. The nitrile group has never been successfully reacted using a transition metal catalyst. This failure is due to the strong affinity between the cyano group and platinum. The general observation is a darkening of the support material indicating the reduction of platinum in the complex to $\mathrm{Pt}(0)[9]$. Therefore, free radical initiation was chosen for this study. Two types of free radical initiators, 
azoisobutyronitrile (AIBN) and tertiary butyl peroxide, were tested as possible catalysts for hydrosilation of the cyano group.

\section{B. High Performance Liquid Chromatography}

Chromatography is an analytical technique in which the components of a mixture are separated on an adsorbent column in a flowing system on the basis of their relative retention. The adsorbent material, or the stationary phase, has taken many forms over the years. The flowing system, or the mobile phase, could be a liquid or a gas. Liquid chromatography is one of the forms of chromatography in which the mobile phase is a liquid [10].

Tswett first introduced liquid chromatography in 1903. However, it started gaining importance in the mid-1970's and quickly improved with the development of column packing materials and on-line detectors. By the 1980's HPLC became the most common technique used for the separation of chemical compounds. Access to extensive computer memories and automation of the equipment further enhanced this technique.

The particle size of the stationary phase was also reduced from $50 \mu \mathrm{m}$ to $3-10 \mu \mathrm{m}$ as an improved method $[10,11]$. The two crucial components of HPLC are the stationary phase and the mobile phase.

\section{Stationary phase}

The solid support present in the column is referred to as the stationary phase which is continuously in contact with the mobile phase. As the sample flows with the mobile phase through the stationary phase, the components of the mixture are eluted according to their non-covalent interactions with the stationary phase. These interactions between 
the solute and the stationary phase are responsible for their separation. The solutes which have stronger interactions with the stationary phase than the mobile phase will elute from the column less quickly, and thus have a longer retention time and vice versa. $[10,11]$

Reverse Phase chromatography operates on the basis of hydrophilicity and lipophilicity. In this type of chromatography, a non-polar stationary phase is used with a more polar mobile phase. The stationary phase usually consists of a silica-based packing with n-alkyl chains covalently attached to it. The more hydrophobic the matrix on the support, the greater is the tendency of the column to retain hydrophobic moieties. Thus, hydrophilic compounds elute more quickly than hydrophobic compounds.

\section{Mobile Phase}

The mobile phase is a liquid that flows continuously through the stationary phase. Basically, it acts as the carrier for the sample. A sample solution is injected into the mobile phase of the chromatographic system through an injector port. The composition of the mobile phase can be altered in order to manipulate the interaction of the sample with the stationary phase. The mobile phase composition can be either isocratic or a gradient, depending on the type of separation. Isocratic elution occurs when the compounds are eluted using a constant mobile phase composition. In gradient elution, the sample is injected while a weaker mobile phase is being applied to the system. The strength of the mobile phase is increased in increments by raising the organic solvent fraction which then results in elution of strongly retained components $[10,11]$. 


\section{Liquid Crystals}

A liquid crystal is a state of matter that is intermediate between a solid crystalline phase and an isotropic liquid phase. The study of liquid crystals began in 1888 when Reinitzer and Lehman made the first observations about this 'mesomorphic' state. The distinctive characteristic of the liquid crystalline state is the tendency of the molecules (mesogens) to point along a common axis, called the director. This is in contrast to molecules in the liquid phase which have no intrinsic order. In the solid state, molecules are highly ordered and have little translational freedom. The characteristic orientational order of the liquid crystal state is between the traditional solid and liquid phases [12]. The alignment of the molecules in different phases is shown in the following diagram (Figure 3).

The tendency of the liquid crystal molecules to point along the director leads to a condition known as anisotropy. This term means that the properties of a material depend on the direction in which they are measured. The anisotropic nature of liquid crystals is responsible for the unique optical properties exploited by scientists and engineers in a variety of applications $[12,13]$

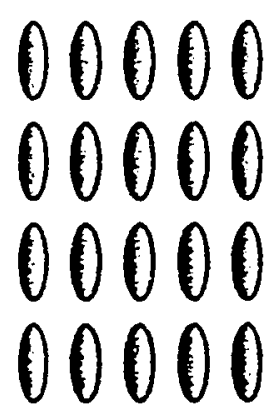

Solid

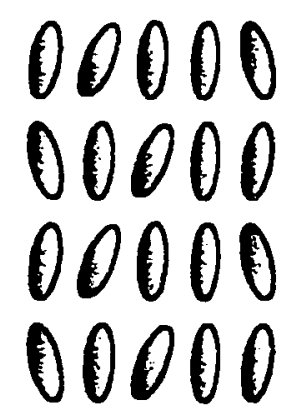

Liquid Crystal

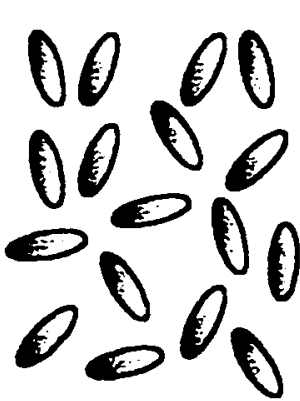

Liquid

Figure 3. Schematic representation of molecular order in different phases [12] 
Some of the more common properties of liquid crystal materials are their rod-like molecular structure, rigidity of the long axis, and strong dipoles and/or easily polarizable substituents. Most liquid crystal compounds exhibit polymorphism or a condition where more than one phase is observed in the liquid crystalline state. The term mesophase is used to describe the "subphases" of liquid crystal materials. Mesophases are formed due to either changes in the temperature or by the influence of solvents. Depending upon the nature of the transition, liquid crystals are broadly classified into two main categories: thermotropic liquid crystals and lyotropic liquid crystals. These two types of liquid crystals are distinguished by the mechanisms that govern their self-organization $[12,13]$.

\section{Thermotropic liquid crystals}

Thermotropic transitions are more common than the lyotropic transitions in liquid crystals. The thermotropic are, as the name suggests, induced thermally. The liquid crystal state can be achieved by raising the temperature of the solid and/or lowering the temperature of a liquid. Depending on the conditions that result in the liquid crystalline state, thermotropic liquid crystals are divided into two categories: enantiomeric liquid crystals and monotropic liquid crystals. The enantiomeric liquid crystal state can be achieved by either lowering the temperature of the liquid or raising the temperature of a solid. The monotropic liquid crystal state can be achieved by either increasing the temperature of a solid or by decreasing the temperature of a liquid but not both $[12,13]$.

\section{Lyotropic liquid crystals}

Lyotropic liquid crystals are formed because of the solvent induced aggregation of the mesogens. The liquid crystals are composed of micellar structures with a lyophilic 
part and a lyophobic part, in the solvent. As the concentration of the solvent increases, along with the cooling of the solution, the size of the micelles increases and subsequently they fuse together. This results in the separation of the liquid crystalline state from the solvent $[12,13]$.

A large number of compounds are known to exhibit one or more liquid crystalline phases. Despite significant differences in their composition, these chemical compounds have some common chemical and physical properties. There are two types of thermotropic liquid crystals: discotics and rod-shaped compounds. Discotics are flat disclike molecules with a core of adjacent aromatic rings. This structure is responsible for the two dimensional columnar arrangement. Rod shaped molecules have an elongated, anisotropic geometry, which allows for preferential alignment along one spatial direction. $[12,13]$

\section{Rod-like Liquid Crystal Phases}

There are many types of liquid crystal states, depending upon the degree of order in the material. This section illustrates the different phases of liquid crystal materials.

\section{a. Nematic Phases}

The nematic liquid crystal phase is characterized by molecules which have no long-range translational order but have a high degree of orientational order. They tend to point in the same direction (along the director). The following diagram (Figure 4) shows that the molecules point vertically but are arranged with no particular order. 


\section{Director}
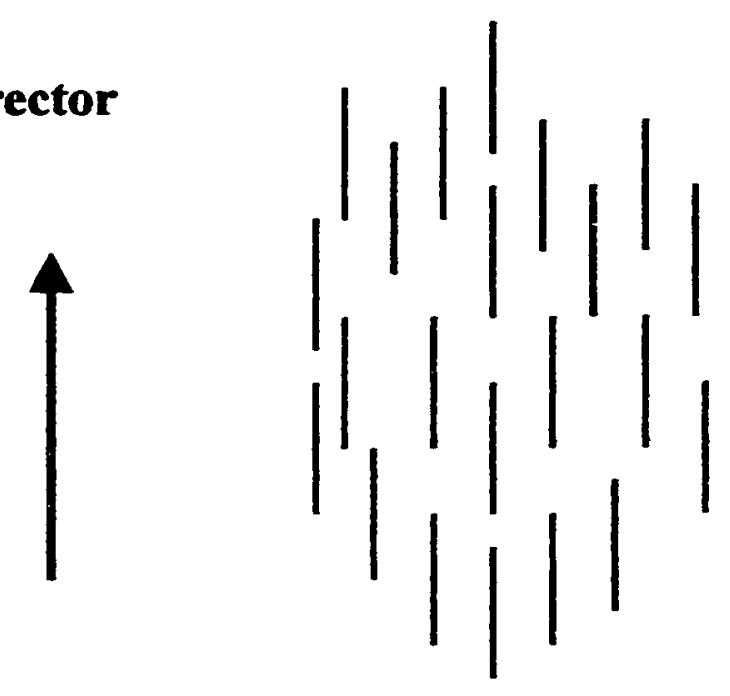

Figure 4. Schematic representation of nematic phase [12]

Liquid crystals are anisotropic materials and the physical properties of the system vary with the average alignment with the director. If the alignment is large, the material is very anisotropic. Similarly, if the alignment is small, the material is almost isotropic [12,13].

\section{b. Cholesteric Phases}

The cholesteric (or chiral nematic) liquid crystal phase is typically composed of nematic mesogenic molecules containing a chiral center which produces intermolecular forces that favor alignment between molecules at a slightly twisted angle to one another. This leads to the formation of a structure similar to a stack of very thin nematic-like layers parallel to the director in each layer which is twisted with respect to those above and below. The directors form a continuous helical pattern about the layer normal to the axis. The following diagram (Figure 5) represents the structure of a cholesteric type of mesophase $[12,13]$. 


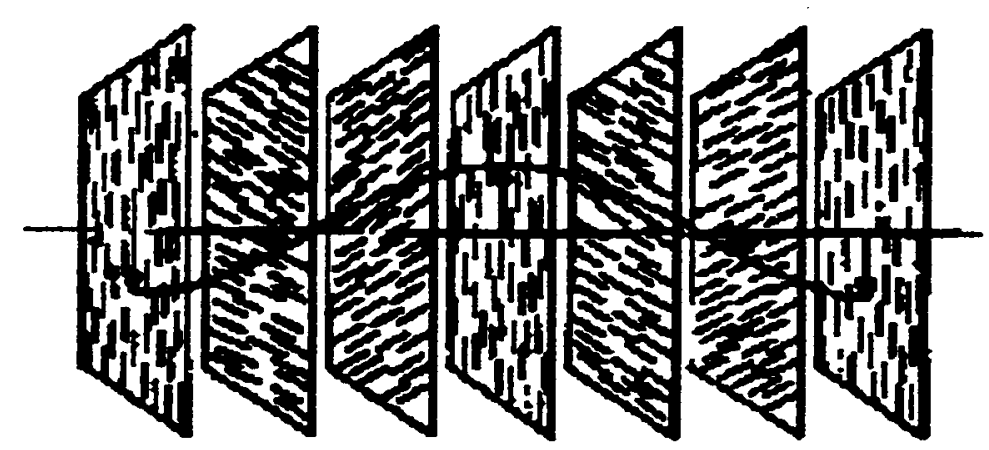

Figure 5. Schematic representation of the cholestric phase [12]

\section{c. Smectic Phases}

The smectic state is another distinct mesophase of liquid crystal substances. Molecules in this phase show a degree of translational order not present in the nematic state. In the smectic state, the molecules maintain the general orientational order of nematics, but also tend to align themselves in layers or planes. Motion is restricted to within these planes, and separate planes are observer to flow past each other. The increased order means that the smectic state is more "solid-like" than the nematic state. Many compounds are observed to form more than one type of smectic phase. As many as 12 of these variations have been identified. However, only the most distinct smectic-A and smectic- $C$ phases are discussed below.

In the smectic-A mesophase (Figure 6), the director is perpendicular to the smectic plane, and there is no particular positional order in the layer $[12,13]$.

In the smectic-C mesophase (Figure 7), molecules are arranged as in the smecticA mesophase, but the director is at a constant tilt angle measured normally to the smectic plane $[12,13]$. 


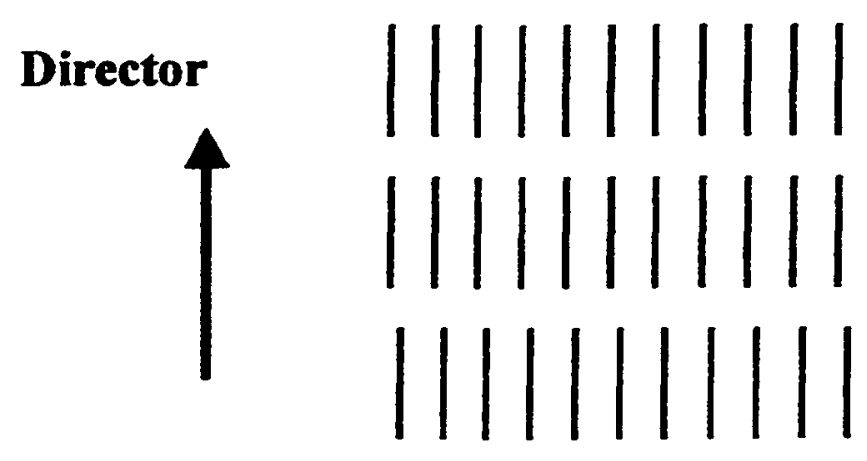

Figure 6. Schematic representation of the smectic $A$ phase [12]

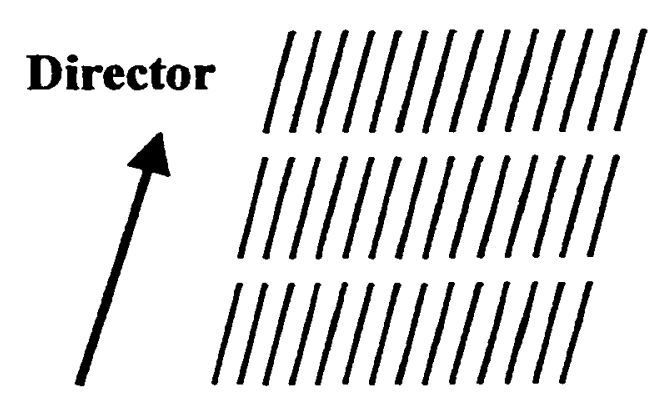

Figure 7. Schematic representation of the smectic C phase [12]

\section{Liquid crystals in HPLC}

Liquid crystal compounds have been used in gas chromatography (GC) for more than 25 years. In GC the liquid crystal compounds are physically adsorbed onto the support material [14]. In HPLC, prolonged exposure to the mobile phase and high-pressure conditions will result in rapid loss of the stationary phase and thus decrease the efficiency of the column. Bonded liquid crystal stationary phase possesses properties that are different from other organic groups attached to silica. These bonded phases exhibit 
unique properties such as high molecular shape selectivity and a higher degree of molecular association between the adjacent ligands. The shape selectivity of the liquid crystal bonded phases could be the result of their mesogenic nature. The liquid crystal bonded phases form ordered "slot" like structures (Figure 8) and the separation occurs due to variable penetration of the solutes into these slots $[15,16]$. These ordered structures are formed due to the association of the individual moieties of the stationary phase. Planar and thin components of the sample will selectively diffuse in and out of these slots over non-planar and bulkier solutes $[15,16]$.

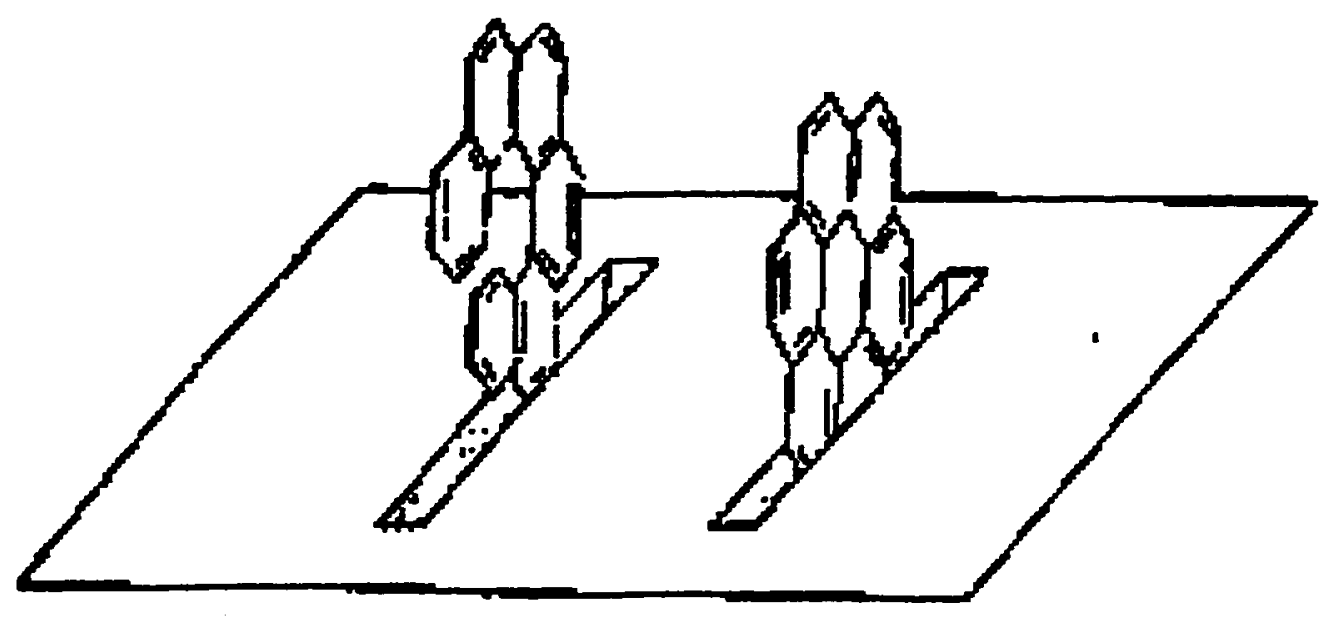

Figure 8. Schematic representation of the "Slot model" [15]

Although, the use of liquid crystal bonded phases for HPLC is a relatively new technique, a variety of chemical compounds have already been separated using these molecules $[17,18]$. The separation of polycyclic aromatic hydrocarbons (PAH) was carried out using a liquid crystal stationary phase by Jinno et al [19]. In another study, cholesteryl 10-undecanoate was used as the stationary phase by Catabay et al, [20]. The cholesteryl phase not only exhibited a typical reverse phase behavior similar to the octadecyl silica phase, but also some specific shape selectivity. This experiment indicated that the cholesteryl phase has the capability of differentiating planar molecules from 
corresponding non-planar molecules for small PAHs [20]. In another study, fat-soluble vitamins were also separated using a liquid crystal bonded phase. However the technique used was supercritical fluid chromatography (SFC) not HPLC [21]. A liquid crystal stationary phase has also been used in SFC for the separation of PAHs [22].

Initially two compounds were selected to determine the reaction conditions that are best for the bonding of liquid crystal compounds with a cyano group to the silica support. Once these were optimized, the same reaction conditions were used for the synthesis of two new liquid crystal bonded phases. The following table lists the different compounds used for this study.

\begin{tabular}{|c|c|c|}
\hline Compound & Molecular formula & MW \\
\hline Cholesteryl-10-undecanoate & $\mathrm{C}_{38} \mathrm{H}_{64} \mathrm{O}_{2}$ & 552.9 \\
\hline 1,2 cyanoethyl pyrrole & $\mathrm{C}_{7} \mathrm{H}_{8} \mathrm{~N}_{2}$ & 120.16 \\
\hline 4-Cyano-4'-N-Pentoxy-1,1'-biphenyl & $\mathrm{C}_{18} \mathrm{H}_{19} \mathrm{NO}$ & 265.36 \\
\hline 4-Cyano-4'-N-Pentyl-1,1'-biphenyl & $\mathrm{C}_{18} \mathrm{H}_{19} \mathrm{~N}$ & 249.02 \\
\hline
\end{tabular}

Table 1. Bonded Moiety

The results of earlier studies on cholesteryl 10- undecanoate [20] indicated that liquid crystal materials are unique in their properties such as molecular shape selectivity. Therefore, it was thought that similar results could be achieved by using other liquid crystal compounds. In this study two rod-like compounds were used to synthesize liquid crystal stationary phases. Their structures are shown below in Figure 9 and Figure 10. 


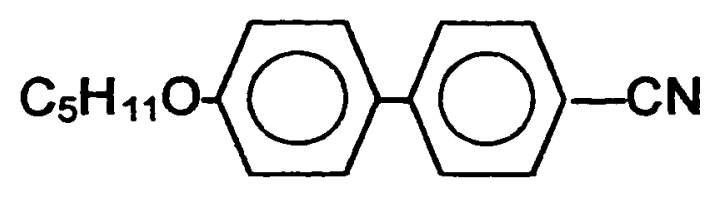

Figure 9. Structure of 4-cyano-4'-n-pentyl-1,1'-biphenyl

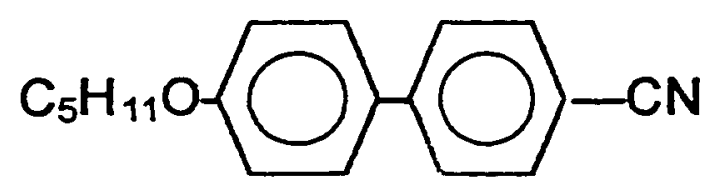

Figure 10. Strueture of 4-cyano-4'-n-pentoxy-1,1'-biphenyl

The rod -like low molar mass (LMM) liquid crystals, such as 4-cyano-4'-npentyl - $1,1^{\prime}$ biphenyl, shown above have an extended conformation which must be maintained through the rigidity and linearity of its constituents. That is, in order for a molecule to display the characteristics of a liquid crystal, it must be rigid and rod-shaped. This is accomplished by the interconnection of two rigid cyclic units. The interconnecting group should cause the resulting compound to have a planar conformation. Linking units containing multiple bonds such as $-\{\mathrm{CH}=\mathrm{N}\}-,-\mathrm{N}=\mathrm{N}-,\{\mathrm{CH}=\mathrm{CH}\}_{\mathrm{n}}-, \mathrm{CH}=\mathrm{N}-\mathrm{N}=\mathrm{CH}-$, etc., are used since they restrict the freedom of rotation. These groups can conjugate with phenyl rings, enhancing the anisotropic polarizability. This linkage increases the molecular length and maintains the rigidity. The liquid crystal bonded phases and the possible reaction schemes are shown below in Figure 11 and Figure 12. 
Bonded Phase Synthesis using free radical initiation<smiles>C#[SiH][CH+]c1ccc(-c2ccc(C#N)cc2)cc1</smiles>

Probable Mechanism

$\equiv \mathrm{Si}-\mathrm{H} \stackrel{\mathrm{FRI}}{\longrightarrow} \mathrm{Si} \bullet+\mathrm{H} \bullet$

$\equiv \mathrm{Si} \cdot+\mathrm{C}_{5} \mathrm{H}_{11} \longrightarrow \bigcirc-\mathrm{CN}$

$\downarrow$

$\mathrm{C}_{5} \mathrm{H}_{11} \bigcirc \bigcirc-\mathrm{CH}_{2} \mathrm{~N}_{\mathrm{Si}}^{\mathrm{Si} \equiv}$

Figure 11. Reaction scheme for 4-cyano-4'-n-pentyl-1,1'-biphenyl bonded phase 
Bonded Phase Synthesis Using Free Radical Initiation<smiles>C=[SiH][CH-][14CH2]Oc1ccc(-c2ccc(C#N)cc2)cc1</smiles>

Probable Mechanism

$\equiv \mathrm{Si}-\mathrm{H} \stackrel{\text { FRI }}{\longrightarrow} \mathrm{Si} \bullet+\mathrm{H} \bullet$

$\equiv \mathrm{Si} \cdot+\mathrm{C}_{5} \mathrm{H}_{11} \mathrm{O} \bigcirc \longrightarrow \longrightarrow \mathrm{CN}$

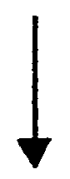

$\mathrm{C}_{5} \mathrm{H}_{11} \mathrm{O} \bigcirc-\mathrm{C}-\mathrm{CH}_{2} \mathrm{Ni}$

Figure 12. Reaction scheme for 4-cyano-4'-n-pentoxy-1,1'-biphenyl bonded phase 


\section{E. Characterization methods}

\section{Fourier Transform Infrared Spectroscopy (FTIR)}

Infrared spectroscopy is a useful method for obtaining structural information of a molecule. It measures the absorption frequencies of certain functional groups that are characteristic for a particular molecule. Hence, it could be used to determine structural information for both organic and inorganic molecules. Using the Fourier transform mode for lower surface area materials enhances the efficiency of this technique. The sensitivity of the technique can be further improved by obtaining the spectrum in the diffuse reflectance mode. In silica, silanols ( $\mathrm{Si}-\mathrm{OH})$ are mainly present on the surface and these are the sites for chemical reactions. The changes in the $\mathbb{R}$ spectrum are very distinct thus revealing the specific surface chemistry. This feature shows that $\mathbb{R}$ is a powerful technique for obtaining qualitative information about different types of materials. IR spectroscopy can be used for the study of both native and modified surfaces [23].

\section{Cross Polarization Magic angle Spinning Nuclear Magnetic Resonance Spectroscopy (CP-MAS NMR)}

NMR is another technique that is extremely useful for obtaining information about the structure and chemical composition of unknown molecules. This technique can be applied to both organic and inorganic molecules for structural characterization [23].

\section{a. ${ }^{13}$ C CP-MAS NMR}

Characterization of modified surfaces such as bonded silica is possible using ${ }^{13} \mathrm{C}$ CP-MAS NMR. This technique is extremely useful in identifying the bonded organic moiety and the success of the bonding process. By analyzing the unbonded compound and the expected chemical shifts, it is possible to even identify individual carbons in the 
bonded moiety. Therefore this technique is useful for confirmation that a chemical compound is either bonded to or adsorbed on the surface of a material. Moreover, it also provides information about relative mobilities of various bonded moieties [23].

\section{b. ${ }^{29}$ Si CP-MAS NMR}

The surface chemistry of oxide surfaces such as silica can be very easily studied using this technique. It provides information about the types of silicon atoms present on both native and bonded silica [23]. Therefore this technique is used to characterize separation materials used as solid supports for HPLC.

\section{Elemental Analysis}

Elemental analysis is one of the useful tools in determining the density of the organic modifier present on the surface. Therefore, it also provides valuable information about the success of bonding. The micro-combustion method is used for determining the carbon percentage of the modified silica surface. The carbon percentage value is then used to determine the surface coverage of silica with the organic modifier.

\section{F. Aims of this study}

The main goal of this study was to synthesize a liquid crystal stationary phase for HPLC. For this project two liquid crystalline compounds, 4-cyano-4'-n-pentyl-1,1'biphenyl and 4-cyano-4'-n-pentoxy-1,1'-biphenyl were chosen. These two samples were chosen because these are the most common compounds used in liquid crystal displays. Reaction conditions such as the presence of different catalysts were investigated to optimize the synthetic procedure. It is expected that under ideal reaction conditions, the liquid crystalline bonded material will be a good candidate for use as a stationary phase in HPLC. 
The modified silica was investigated using different characterization techniques. These techniques include DRIFT, ${ }^{13} \mathrm{C}$ and ${ }^{29} \mathrm{Si} \mathrm{CP}-\mathrm{MAS}$ NMR, and elemental analysis. These methods were used to determine the success of bonding and the surface coverage of these liquid crystal compounds on silica. The chromatographic studies were designed to test separations based on reverse-phase conditions as well as molecular shape selectivity, which is unique to liquid crystalline stationary phases. Perkin-Elmer test mixture and polycyclic aromatic hydrocarbons were used respectively to analyze the reverse-phase behavior and molecular shape selectivity of the stationary phase. 


\section{CHAPTER I - EXPERIMENTAL}

\section{A. Materials}

\section{Chemicals}

The CAS registry numbers of all the chemicals used are listed in Table 2.

\begin{tabular}{|l|l|}
\hline Chemical Name & CAS Registry Number \\
\hline Anthracene & {$[120-12-7]$} \\
\hline $2,2-$ azobisisobutyronitrile & {$[4187-87-5]$} \\
\hline Benzene & {$[71-43-2]$} \\
\hline Clonazepam & {$[1622-61-3]$} \\
\hline Cholesteryl 10-undecanoate & {$[30948-01-7]$} \\
\hline 1,2-Cyanoethylpyrrole & $43036-06-2$ \\
\hline 4-Cyano-4'-n-pentyl-1,1' ${ }^{\prime}$ - biphenyl & $40817-08-1$ \\
\hline 4-Cyano-4'-n-pentoxy-1,1'- biphenyl & $52364-71-3$ \\
\hline Diazepam & {$[439-14-5]$} \\
\hline Diethyl ether & {$[60-29-7]$} \\
\hline Hexachloroplaticinic acid(38-40\% Pt) & {$[16941-12-1]$} \\
\hline Hydrochloric acid & {$[7647-01-0]$} \\
\hline Methanol & {$[67-56-1]$} \\
\hline Methylene chloride & {$[75-09-2]$} \\
\hline
\end{tabular}




\begin{tabular}{|l|l|}
\hline Nitrazepam & {$[146-22-5]$} \\
\hline Oxazepam & {$[604-75-1]$} \\
\hline Potassium Bromide & {$[7758-02-3]$} \\
\hline Sodium hydroxide & {$[1310-73-2]$} \\
\hline Temazepam & {$[846-50-4]$} \\
\hline Tetrahydrofuran & {$[109-99-8]$} \\
\hline Tertiary butyl peroxide & {$[110-05-4]$} \\
\hline Triethoxysilane (TES) & {$[998-30-1]$} \\
\hline Toluene & {$[108-88-3]$} \\
\hline Wilkinson's catalyst & {$[14694-95-2]$} \\
\hline
\end{tabular}

Table 2. CAS Registry numbers of the chemicals used

2.Chemicals for bonded phase synthesis

All the chemicals used for the bonded phase syntheses were reagent grade.

Cholesteryl 10-undecanoate was purchased from Sigma (St. Louis, MO).

1,2-cyanoethylpyrrole was purchased from Aldrich (Milwaukee, WI).

4 -cyano-4'-n-pentyl-1, $1^{\prime}$-biphenyl and 4 -cyano-4'-n-pentoxy-1, $1^{\prime}$-biphenyl were purchased from EM industries (Hawthorne, NY). All the solvents, diethyl ether (Fisher Scientific, Fair Lawn, NJ), dioxane (Fisher Scientific, Fair Lawn, NJ), methylene chloride, tetrahydrofuran, (J.T. Baker Chemicals Co. Phillipsburg, NJ) and tolulene (EM Science, Gibstown, NJ) were obtained in reagent grade. All the solvents were used as received. Hexachloroplatinic acid hydrate was purchased from Strem Chemicals (Newbury Port, MS). Tertiary butyl peroxide and azobisisobutyronitrile (AIBN) were obtained from Aldrich (Milwaukee, WI). Chlorotris(triphenylphosphine) rhodium 
(Wilkinson's catalyst) was purchased from Strem Chemicals, Inc (Newburyport, MA).

The silica gels, Vydac TP 106 lot \# 930816-4A and Kromasil lot \# AT 0112 were obtained from The Separations Group (Hesperia, CA) and Eka Nobel (Bohus, Sweden) respectively. Vydac TP 106 silica has a particle size of $6.5 \mu$, a pore diameter of $300 \mathrm{~A}^{\circ}$ and a surface area of $106 \mathrm{~m}^{2} / \mathrm{g}$. Kromasil silica has a particle size of $5 \mu$, a pore diameter of $100 \mathrm{~A}^{\circ}$ and a surface area of $340 \mathrm{~m}^{2} / \mathrm{g}$. Triethoxysilane (TES) was obtained from Huls Petrarch Systems (Huls, Bristol, PA).

\section{B. Bonded phase synthesis}

All the glassware used for the synthesis was properly washed first with tap water and mild soap and then finally with deionized water and dried overnight at $110^{\circ} \mathrm{C}$.

\section{Synthesis of the silica hydride intermediate via the TES Silanization process}

The preparation of the silica hydride intermediate was carried out according to a method previously described by Chu et al. [5]. About $10 \mathrm{~g}$ of Silica (Vydac TP 106) was dried under vacuum at $110^{\circ} \mathrm{C}$ for $24 \mathrm{~h}$. The preparation of the TES solution was carried out in a glove-box thoroughly purged with nitrogen. The glove-box was first purged alternatively with nitrogen and vacuum five times. Triethoxysilane $(8.5 \mathrm{~mL}$ of $1.00 \mathrm{mM})$ was measured in a $10 \mathrm{~mL}$ graduated cylinder and transferred to a $50 \mathrm{~mL}$ volumetric flask. The flask was then filled with distilled 1,4-dioxane up to the mark. The TES solution was transferred to a $125 \mathrm{~mL}$ addition funnel. Outside the glove-box, a $500 \mathrm{~mL}$, three-necked round bottom flask was equipped with a West condensor, addition funnel (with the TES solution), a thermometer, a glass stopper, magnetic stirring bar and a heating mantle. Ten grams of Vydac TP 106 silica were added to the round bottom flask followed by $245 \mathrm{~mL}$ of distilled 1,4-dioxane. To this mixture $10 \mathrm{~mL}$ of $2.3 \mathrm{M} \mathrm{HCl}$ were added and the reaction 
solution was heated to reflux at $93^{\circ} \mathrm{C}$ for an hour. All the joints were properly sealed with parafilm. This was followed by drop-wise addition of the TES solution for about 25 to 30 minutes. The reaction mixture was then heated at reflux for another hour.

Once the reaction was complete and the apparatus was cooled to room temperature, the mixture was allowed to settle and the p-dioxane was decanted. The remaining paste was distributed equally into four centrifuge tubes (IEC-HNS) along with a small amount of toulene reagent. The silica hydride mixture in toulene was then stirred for 10 minutes and centrifuged at approximately $1000 \mathrm{~g}$ forces (1500 rpm) (Damon, IEC div. Model, HN-S, Needham Heights, MA) for another 10 minutes. The supernatant solution was decanted. The washing procedure was done in the following order: three times ( $5 \mathrm{~mL}$ each) with a freshly prepared $1: 1(\mathrm{v} / \mathrm{v})$ tetrahydrofuran (THF)-water mixture, two times ( $5 \mathrm{~mL}$ each) with pure $\mathrm{THF}$ and finally with diethylether $(5 \mathrm{~mL})$. The silica hydride was transferred into a beaker, covered with a watch glass and dried overnight in the hood at room temperature. The silica hydride was dried under vacuum at $110^{\circ} \mathrm{C}$ for another $24 \mathrm{~h}$. The product was then stored in an airtight container.

\section{Preparation of Speier's catalyst}

The preparation of Speier's catalyst was performed in a glove-box thoroughly purged with nitrogen. In this procedure, $0.4 \mathrm{~g}$ of chloroplatinic acid hexahydrate was added to $100 \mathrm{~mL}$ of isopropanol at $93^{\circ} \mathrm{C}$ [6]. The catalyst (a $10 \mathrm{mM}$ solution) was stored in the refrigerator. 


\section{Kromasil silica hydride synthesis}

All the glassware used for the synthesis was properly washed with deionized water and dried ovemight at $110^{\circ} \mathrm{C}$ for this procedure. About $3 \mathrm{~g}$ of silica (Kromasil lot \# AT0112) was dried under vacuum at $110^{\circ} \mathrm{C}$ for $24 \mathrm{hr}$.

The preparation of the TES solution was carried out as described above for Vydac silica hydride. The synthesis was carried out in a $500 \mathrm{~mL}$, three-necked round bottom flask equipped with a West condensor, thermometer, glass stopper, addition funnel (with the TES solution) and magnetic stirring bar. To the round bottom flask, $245 \mathrm{~mL}$ of 1,4 -dioxane followed by $3 \mathrm{~g}$ of Kromasil silica and $10 \mathrm{~mL}$ of $2.3 \mathrm{M} \mathrm{HCl}$ were added. The reaction mixture was heated to reflux at $93^{\circ} \mathrm{C}$ for an hour. This was followed by the drop-wise addition of TES ( $45 \mathrm{~mL}$ of a $1.0 \mathrm{M}$ ) solution for $25-30$ minutes. When all of the TES solution was added, the reaction mixture was heated to reflux at $93^{\circ} \mathrm{C}$ for another hour.

The apparatus was then allowed to cool to room temperature. The supernatant was decanted and the product was washed several times. The washing procedure was identical to the one followed for Vydac silica hydride preparation. The final product, Kromasil silica hydride, was then transferred into a beaker, covered with a watch-glass and dried in the hood overnight. It was then dried under vacuum at $110^{\circ} \mathrm{C}$ for 24 hours. The dry silica hydride was stored in an airtight vial.

\section{Bonded phase synthesis}

\section{a. Hydrosilation with Speier's catalyst}

The bonded phase synthesis was carried out according to a method previously described by Chu et al. [5]. About $7.5 \mathrm{~mL}$ of distilled toulene was transferred to a $50 \mathrm{~mL}$ 
three necked flask, equipped with a $25 \mathrm{~cm}$ West condensor, thermometer, glass stopper, magnetic stir bar and a $50 \mathrm{~mL}$ heating mantle. Cholesteryl 10 -undecanoate $(0.836 \mathrm{~g})$ was added to the three-necked flask while stirring. To this solution ( $0.2 \mathrm{M}$ cholesteryl $10-$ undecanoate), $0.6 \mathrm{~mL}$ of $1 \mathrm{mM}$ Speier's catalyst was added and the mixture was heated to reflux at a constant temperature of $100 \pm 2{ }^{\circ} \mathrm{C}$ for about an hour. Silica hydride (Vydac TP $106,0.5 \mathrm{~g}$ ) was added to the above mixture and the flask was flushed with nitrogen. All the joints were properly sealed with parafilm and the mixture was refluxed at $100^{\circ} \mathrm{C}$ for about 96 hours. The resultant product was transferred to a centrifuge tube and centrifuged. The supernatant was discarded and the remaining paste was washed with 5 $\mathrm{mL}$ portions of several solvents. This process included washing the product three times $(5$ $\mathrm{mL}$ each) with toluene, two times with methylene chloride ( $5 \mathrm{~mL}$ each) and twice $(5 \mathrm{~mL})$ with diethyl ether. The final product (bonded phase) was dried in the hood at room temperature overnight and under vacuum at $110^{\circ} \mathrm{C}$ for another $24 \mathrm{~h}$. The bonded phase was then analyzed using DRIFT, NMR spectroscopy and elemental analysis to determine the success of bonding.

\section{b. Hydrosilation with Wilkinson's catalyst}

All the relevant glassware was washed with deionized water and dried in the oven at $110^{\circ} \mathrm{C}$ overnight. About $7.5 \mathrm{~mL}$ of toluene was transferred to a $50 \mathrm{~mL}$ three necked round bottom-flask equipped with a thermometer, $25 \mathrm{~cm}$ West condensor, glass stopper, magnetic stir bar and a $50 \mathrm{~mL}$ heating mantle. 1,2 cyanoethyl pyrrole $(0.2 \mathrm{~mL})$ and 0.651 $\mathrm{g}$ (in toulene) of Wilkinson's catalyst were then added to the round bottom flask. The mixture was then heated to reflux at $100^{\circ} \mathrm{C} \pm 2{ }^{\circ} \mathrm{C}$ for about an hour. Kromasil silica hydride $(0.5 \mathrm{~g})$ was then added to the above mixture. The flask was flushed with nitrogen 
and all the joints were properly sealed. The mixture was refluxed at $100^{\circ} \mathrm{C}$ for $96 \mathrm{~h}$. The resultant product was then centrifuged and washed as described earlier for cholesteryl 10 -undecanoate bonded phase synthesis. The final product was dried overnight at room temperature and then under the vacuum at $100^{\circ} \mathrm{C}$ for $24 \mathrm{~h}$.

\section{Free radical hydrosilation}

\section{a. 1,2-Cyanoethylpyrrole bonded phase}

Hydrosilation of the silica hydride surface by free radical addition was carried out according to a method previously described by Pesek et al. [4]. Azobisisobutyronitrile (AIBN) and tertiary butyl peroxide were used as the free radical initiators for this experiment. A mixture of $10 \mathrm{~mL}$ of distilled toulene, $1 \mathrm{~mL}$ of 1,2 -cyanoethylpyrrole and $40 \mu \mathrm{L}$ of t-butyl peroxide (or $\sim 0.05 \mathrm{~g}$ of $\mathrm{AIBN}$ ) were added to a $25 \mathrm{~mL}$ three necked round bottom flask. The equipment setup was the same as described above. The mixture in the round-bottom flask was heated at a constant temperature of $60-70^{\circ} \mathrm{C}$ for about an hour while stirring. Kromasil silica hydride $(0.5 \mathrm{~g})$ was added slowly through the open neck of the flask. The flask was flushed with nitrogen and all the joints were sealed properly. The temperature of the reaction mixture was then raised to $100^{\circ} \mathrm{C} \pm 2{ }^{\circ} \mathrm{C}$. The reaction was allowed to proceed for about $100 \mathrm{~h}$. The final product was centrifuged and the supernatant decanted. Toulene, methylene chloride and diethyl ether were used to wash the product. The washing procedure was the same as described above for cholesteryl 10-undecanoate. 


\section{b. 4-Cyano-4'-n-pentyl-1,1'-biphenyl bonded silica}

A similar procedure was carried out as described in Section 5a for this bonded phase synthesis. In this case, $1 \mathrm{~mL}$ of 4-cyano-4'-n-pentyl-1, 1'-biphenyl was reacted with $0.5 \mathrm{~g}$ of Kromasil silica hydride in $10 \mathrm{~mL}$ of toluene. Tertiary butyl peroxide $(40 \mu \mathrm{L})$ was used as the free radical initiator. The same procedure was repeated for Vydac TP 106 silica hydride.

\section{c. 4-Cyano-4'-n-pentyl-1,1'-biphenyl bonded silica}

The procedure was the same as described in Section 5a, except that the liquid crystal compound used was 4-cyano-4'-n-pentoxy-1,1'-biphenyl. This liquid crystal compound was reacted with $0.5 \mathrm{~g}$ of Kromasil silica hydride in $10 \mathrm{~mL}$ toluene in the presence of the free radical initiator t-butyl peroxide $(40 \mu \mathrm{L})$. The same procedure was repeated for Vydac TP 106 silica hydride.

\section{Column Packing}

4-cyano-4'-n-pentoxy-1,1'-biphenyl bonded silica (Vydac TP 106) was packed in a stainless steel column $(15 \mathrm{~cm} \times 4.6 \mathrm{~mm}$ internal diameter, Alltech Associates Inc., Deerfield, IL). HPLC grade methanol was used as the driving solvent. Approximately 1.8 $\mathrm{g}$ of bonded silica was added to a $10 \%(\mathrm{v} / \mathrm{v})$ methanol in chloroform and the mixture was sonicated for 10 minutes. The slurry was packed into the column under high pressure (6000 psi) using nitrogen. The packing was followed by a relaxation period of about 30 minutes before the column was removed from the apparatus (Haskell pump; Haskell, Burbank, CA). The 4-cyano-4'-n-pentyl-1,1'-biphenyl bonded silica was sent to Higgins Analytical, Inc (Mountain View, CA) for packing. It was packed into a shorter column $(50 \times 4.6 \mathrm{~mm})$ because a longer column resulted in too high a back-pressure. 


\section{Instrumentation}

\section{Surface Characterization Techniques}

\section{a. Diffuse Reflectance Infrared Fourier Transform Spectroscopy (DRIFT)}

A Perkin-Elmer Model 1800 FT-IR spectrophotometer (Perkin-Elmer, Norwalk, CT) was used for acquiring IR spectra. The IR spectra of all the samples were taken in the diffuse-reflectance mode. The diffuse reflectance accessory was obtained from Spectra Tech., Stamford, CT. It consists of a $2 \mathrm{~mm}$ deep sample cup with a diameter of $2 \mathrm{~mm}$. Nitrogen was used to purge (55-60 psi) the sample compartment of any moisture present. One part of the sample was mixed with one part of ground $\mathbb{R}$-grade $\mathrm{KBr}(50 \%$ dilution) and was placed in the sample cup. $\mathrm{KBr}$ was used as the reference material. Each spectrum was scanned 200 times at a resolution of $2 \mathrm{~cm}^{-1}$ over a range of $4000-450 \mathrm{~cm}^{-1}$. The computer system (Perkin-Elmer model 7500) along with the plotter (Hewlett Packard 7475 A) were used to process the signal and plot the spectra.

A Mattson Infrared Infinity series FT-IR spectrometer was also used for obtaining the IR spectra of some of the samples. This instrument was interfaced with Winfirst software loaded on a digital Venturis Fx-2 series computer system. A similar procedure was carried out as explained above, except that the sample was diluted only $5 \%$ with $\mathrm{KBr}$ as compared to $50 \%$ used on the Perkin-Elmer instrument. 


\section{b. Cross-polarization magic-angle spinning nuclear magnetic resonance spectroscopy (CP-MAS NMR)}

Both ${ }^{13} \mathrm{C}$ and ${ }^{29} \mathrm{Si}$ CP-MAS NMR spectra were obtained on a Bruker (Billerica, MA) MSL 300 spectrometer. The spectra were obtained by placing the solid samples, approximately $200 \mathrm{mg}$, in a rotor spinning at $4700-5200 \mathrm{~Hz}$. The spectra were obtained with $3 \mathrm{~ms}$ and $5 \mathrm{~ms}$ contact times for ${ }^{13} \mathrm{C}$ and ${ }^{29} \mathrm{Si}$ respectively. The repetition rate was $5 \mathrm{sec}$ for both the nuclei. Glycine and polyhydridosiloxane were used as the external references for ${ }^{13} \mathrm{C}$ and ${ }^{29} \mathrm{Si}$ respectively.

Some samples were sent to Karl Eberhard at the University of Tubingen, Germany for analysis. The sample 4-cyano-4'-n-pentoxy-1, 1'-biphenyl was run with a 4 $\mathrm{mm}$ rotor at a frequency of $10000 \mathrm{~Hz}$. Cholesteryl 10-undecanoate was run with a $7 \mathrm{~mm}$ rotor at a frequency of $4000 \mathrm{~Hz}$.

\section{High Performance Liquid Chromatography}

The system consisted of a Perkin-Elmer (Norwalk, CT) Series 200 LC HPLC pump, a Perkin-Elmer model LC 295 UV-VIS multi-wavelength detector and a Hewlett Packard Model 3395 integrator. The solvents were degassed thoroughly using helium gas at a flow rate of $50 \mathrm{~mL} / \mathrm{min}$ before the analysis. The helium flow was then reduced to $5 \mathrm{~mL} / \mathrm{min}$ during analysis to prevent the formation of any gas bubbles. Potassium nitrate was used to obtain the void volume.

A Millipore apparatus was used to filter water. The National Institute of Standards and Technology (NIST, Gaithersburg, MD) donated the standard reference mixture, SRM 869. The Perkin-Elmer universal mixture was obtained from Perkin-Elmer (Norwalk, CT). Benzodiazepines were obtained from Hoffman-La Roche, Switzerland. 


\section{Elemental Analysis}

All the bonded phases were sent to Desert Analytics (Tuscon, AZ) for the determination of the carbon content in each sample. A conventional combustion method was used for the analysis. The elemental analysis results were then used to calculate the surface coverage of the bonded phases. The surface coverage values are presented in the Results section.

\section{Surface Coverage}

The elemental analysis results were used to calculate the surface coverage, $\alpha_{R}$, of the modified silica. The following equation was used for this calculation [24].

$$
\alpha_{R}\left(\mu \mathrm{mol} / \mathrm{m}^{2}\right)=\frac{10^{6} \mathrm{P}_{\mathrm{c}}}{\left(100 \mathrm{Mn}_{\mathrm{c}}-\mathrm{P}_{\mathrm{d}} \mathrm{M}_{\mathrm{R}}\right) \mathrm{S}_{\mathrm{BET}}}
$$

Where $P_{c}$ is the carbon percentage by weight of the modified silica. $n_{c}$ is the number of carbon atoms present in the bonded phase, $M_{c}$ is the atomic weight of carbon and $M_{R}$ is the molecular weight of the organic compound. BET refers to the nitrogen absorption method developed by Brunauer, Emmet and Teller to determine the surface area. $S_{B E T}$ is the specific surface area $\left(\mathrm{m}^{2} / \mathrm{g}\right)$ of the silica substrate. The maximum surface coverage value that can be calculated is about $8 \mu \mathrm{mol} / \mathrm{m}^{2}$. This value represents the average amount of silanols present on the silica surface [24].

\section{E. Hydrolytic Stability Test}

Bonded silica (100 mg) was placed into a centrifuge tube along with $5 \mathrm{~mL}$ of $10 \mathrm{mM} \mathrm{HCl}(\mathrm{pH} \mathrm{2.02)}$ in $10 \%$ methanol. The mixture was stirred for 24 hours at room temperature. The mixture was then centrifuged and the supernatant was discarded. The 
remaining solid was washed three times ( $5 \mathrm{~mL}$ each) with toulene, followed by three times with methylene chloride ( $5 \mathrm{~mL}$ each) and finally twice ( $5 \mathrm{~mL}$ each) with diethyl ether. The resultant product was then dried in a hood overnight and then under vacuum at $\left(110^{\circ} \mathrm{C}\right)$ for another 24 hours. For base hydrolysis, $5 \mathrm{~mL}$ of $10 \mathrm{mM} \mathrm{NaOH}(\mathrm{pH} 9)$ in $10 \%$ methanol was used. The same procedure used in acid hydrolysis was followed. The resulting solid samples were investigated using DRIFT. 


\section{CHAPTER III - RESULTS AND DISCUSSION}

\section{A. Elemental Analysis}

Table 3 shows the surface coverage values of all the bonded phases synthesized. The surface coverage was calculated from the carbon percentage according to equation (1).

\begin{tabular}{|c|c|c|c|c|c|c|}
\hline Bonded phase & $\mathbf{M w}$ & Silica & $\begin{array}{l}S_{\text {bet }} \\
\mathrm{m}^{2} / g\end{array}$ & Catalyst & $\% \mathrm{C}$ & $\begin{array}{c}\text { Surface } \\
\text { coverage } \\
\mu \mathrm{mol} / \mathrm{m}^{2}\end{array}$ \\
\hline $\begin{array}{l}\text { Cholestereyl - 10- } \\
\text { undecanoate }\end{array}$ & 552.9 & $\begin{array}{l}\text { Vydac } \\
\text { TP }\end{array}$ & 106 & $\begin{array}{l}\text { Hexachloro } \\
\text { platinic acid }\end{array}$ & 3.6 & 0.76 \\
\hline $\begin{array}{c}\text { 1,2-Cyanoethyl } \\
\text { pyrrole }\end{array}$ & 120.16 & Kromasil & 340 & Wilkinson's & 15.1 & 6.7 \\
\hline $\begin{array}{c}\text { 1,2-Cyanoethyl } \\
\text { pyrrole }\end{array}$ & 120.16 & Kromasil & 340 & $\overline{A I B N}$ & 3.6 & 1.3 \\
\hline $\begin{array}{c}\text { 1,2-Cyanoethyl } \\
\text { pyrrole }\end{array}$ & 120.16 & Kromasil & 340 & $\begin{array}{l}\text { t- butyl } \\
\text { peroxide }\end{array}$ & 8.9 & 3.6 \\
\hline $\begin{array}{c}\text { 4-Cyano-4'-n-pentoxy- } \\
\text { 1,1'-biphenyl }\end{array}$ & 265.36 & Kromasil & 340 & $\begin{array}{l}\text { t- butyl } \\
\text { peroxide }\end{array}$ & 2.7 & 0.38 \\
\hline $\begin{array}{c}\text { 4-Cyano-4'-n-pentyl- } \\
\text { 1,1'-biphenyl }\end{array}$ & 249.02 & Kromasil & 340 & $\begin{array}{l}\text { t-butyl } \\
\text { peroxide }\end{array}$ & 3.9 & 0.56 \\
\hline $\begin{array}{c}\text { 4-Cyano-4'-n-pentoxy- } \\
1,1^{\prime} \text {-biphenyl }\end{array}$ & 265.36 & $\begin{array}{l}\text { Vydac } \\
\text { TP }\end{array}$ & 106 & $\begin{array}{l}\text { t-butyl } \\
\text { peroxide }\end{array}$ & 1.9 & 0.84 \\
\hline $\begin{array}{c}\text { 4-Cyano-4'-n-pentyl- } \\
1,1^{\prime} \text {-biphenyl } \\
\end{array}$ & 249.02 & $\begin{array}{c}\text { Vydac } \\
\text { TP }\end{array}$ & 106 & $\begin{array}{l}\text { t- butyl } \\
\text { peroxide }\end{array}$ & 2.0 & 0.88 \\
\hline
\end{tabular}

Table 3. Elemental Analysis 


\section{B. Characterization of Silica Hydride}

\section{DRIFT spectra}

The DRIFT spectra for Kromasil silica hydride and Vydac silica hydride are shown in Figure 13 and Figure 14 respectively. These spectra show an intense Si-H bond stretching at $2250 \mathrm{~cm}^{-1}$ for both Kromasil silica hydride and Vydac silica hydride. These results confirm the success of the TES silanization reaction.

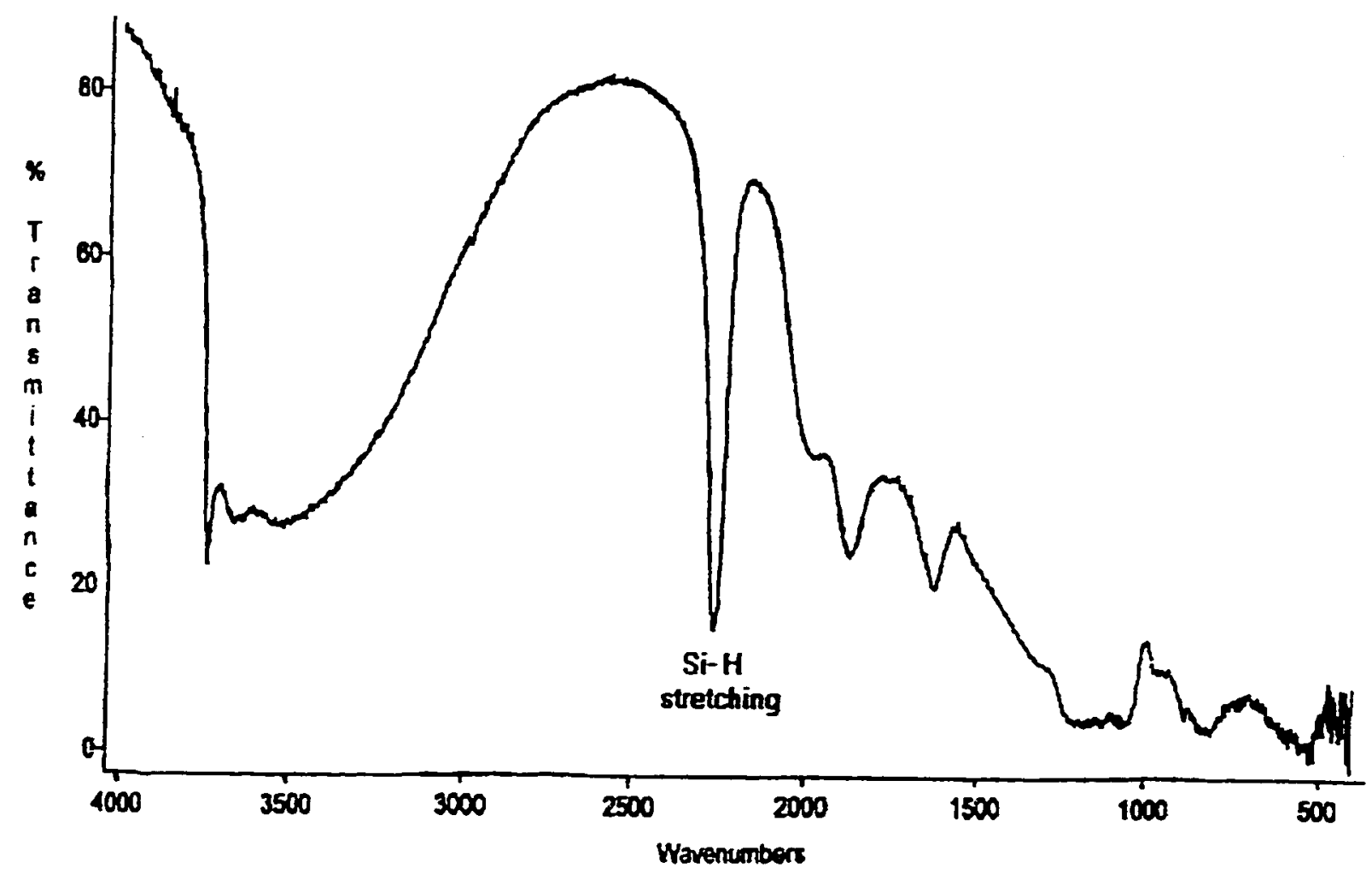

Figure 13. DRIFT spectrum of Kromasil silica hydride 


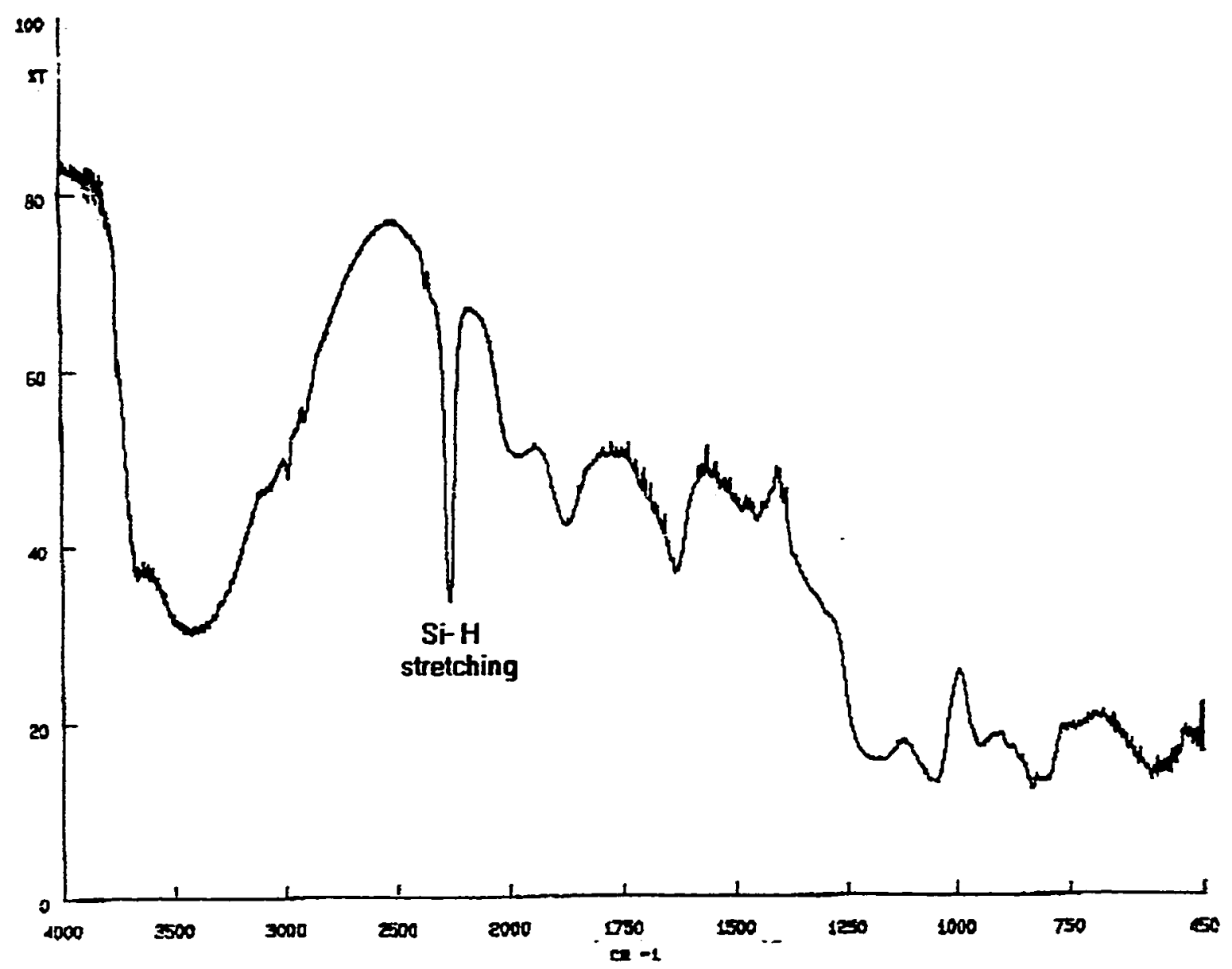

Figure 14. DRIFT spectrum of Vydac silica hydride 


\section{$2{ }^{29}$ Si CP-MAS NMR spectra}

Figure 15 and Figure 16 show the ${ }^{29}$ Si CP-MAS NMR spectra for Kromasil silica hydride and Vydac silica hydride respectively. The peak around $85 \mathrm{ppm}$ is due to a silicon atom bonded to a hydride and three siloxane linkages and the peak near 75 ppm represents a silicon atom bonded to a hydroxide, a hydride and two siloxane linkages. The peak at $110 \mathrm{ppm}$ is due to the presence of silicon atoms in the siloxane backbone, i.e., silicon atoms with four siloxane linkages. The peak at $100 \mathrm{ppm}$ is due to the silicon atoms with three siloxane linkages and a hydroxyl group [3]. These spectra also confirm the success of the TES silanization reaction.

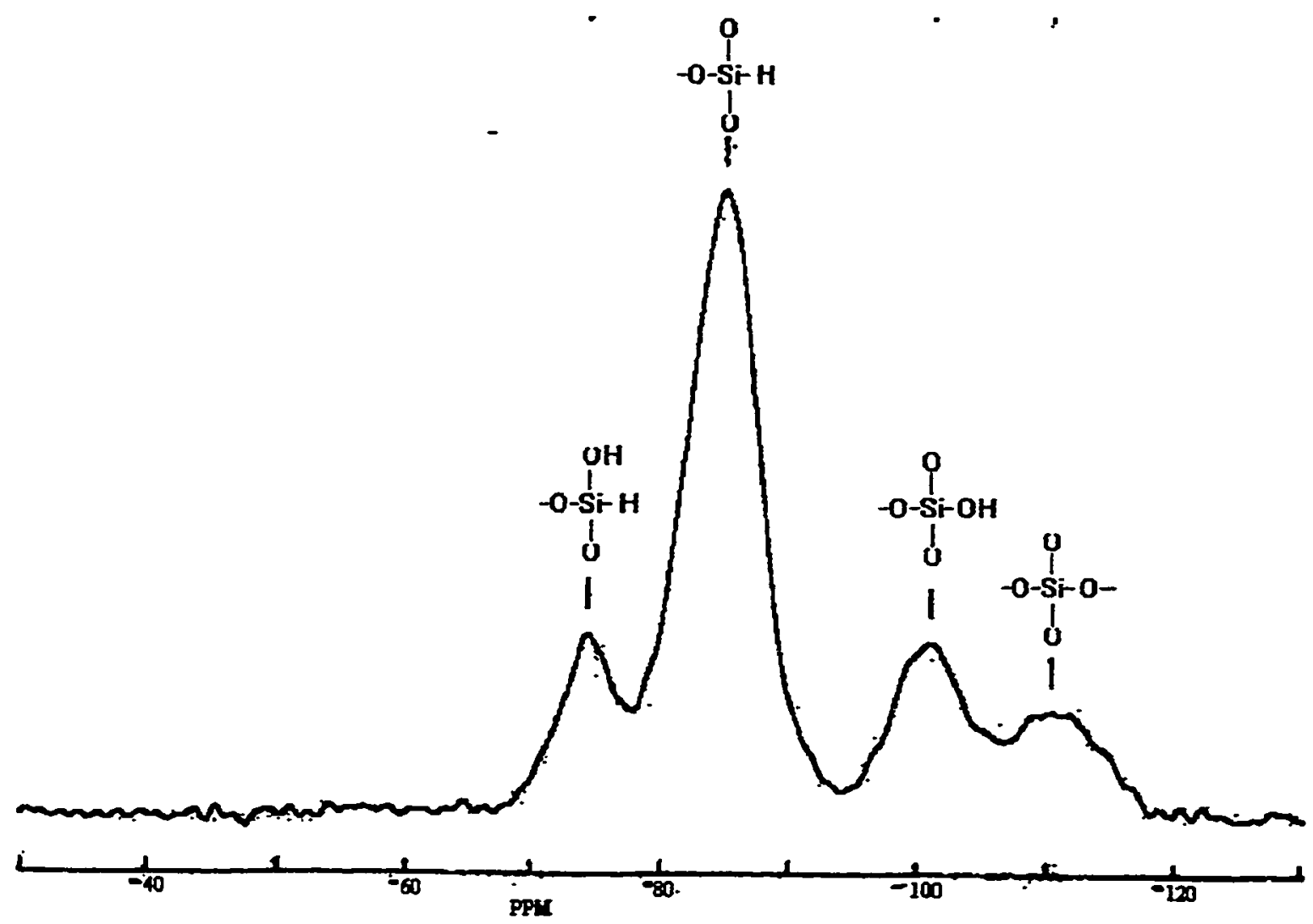

Figure 15. The ${ }^{29}$ Si CP-MAS NMR spectrum of Kromasil silica hydride 


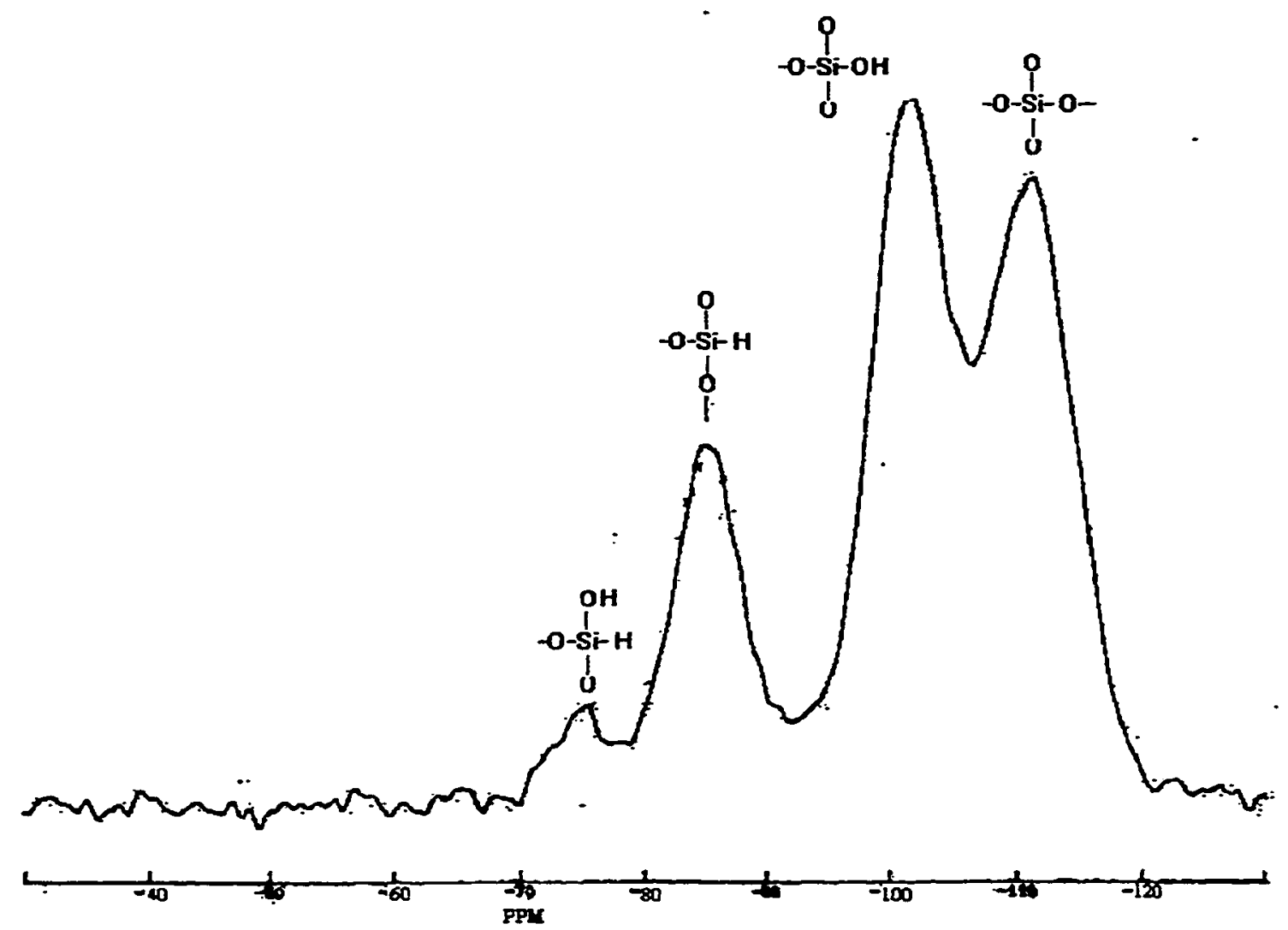

Figure 16. The ${ }^{29}$ Si CP-MAS NMR spectrum of Vydac silica hydride 


\section{Cholesteryl 10-undecanoate bonded phase characterization}

\section{DRIFT spectrum}

The bonded phase cholesteryl 10 -undecanoate was used as the test material for this study. The DRIFT spectrum of cholesteryl 10-undecanoate bonded to silica hydride is shown in Figure 17. This figure shows that the intensity of the Si-H stretching band at $2250 \mathrm{~cm}^{-1}$ (Figure 14) is reduced after bonding. This feature in conjunction with $\mathrm{C}-\mathrm{H}$ stretching bands in the range of $2800-3000 \mathrm{~cm}^{-1}$ indicate the success of bonding the liquid crystal moiety.

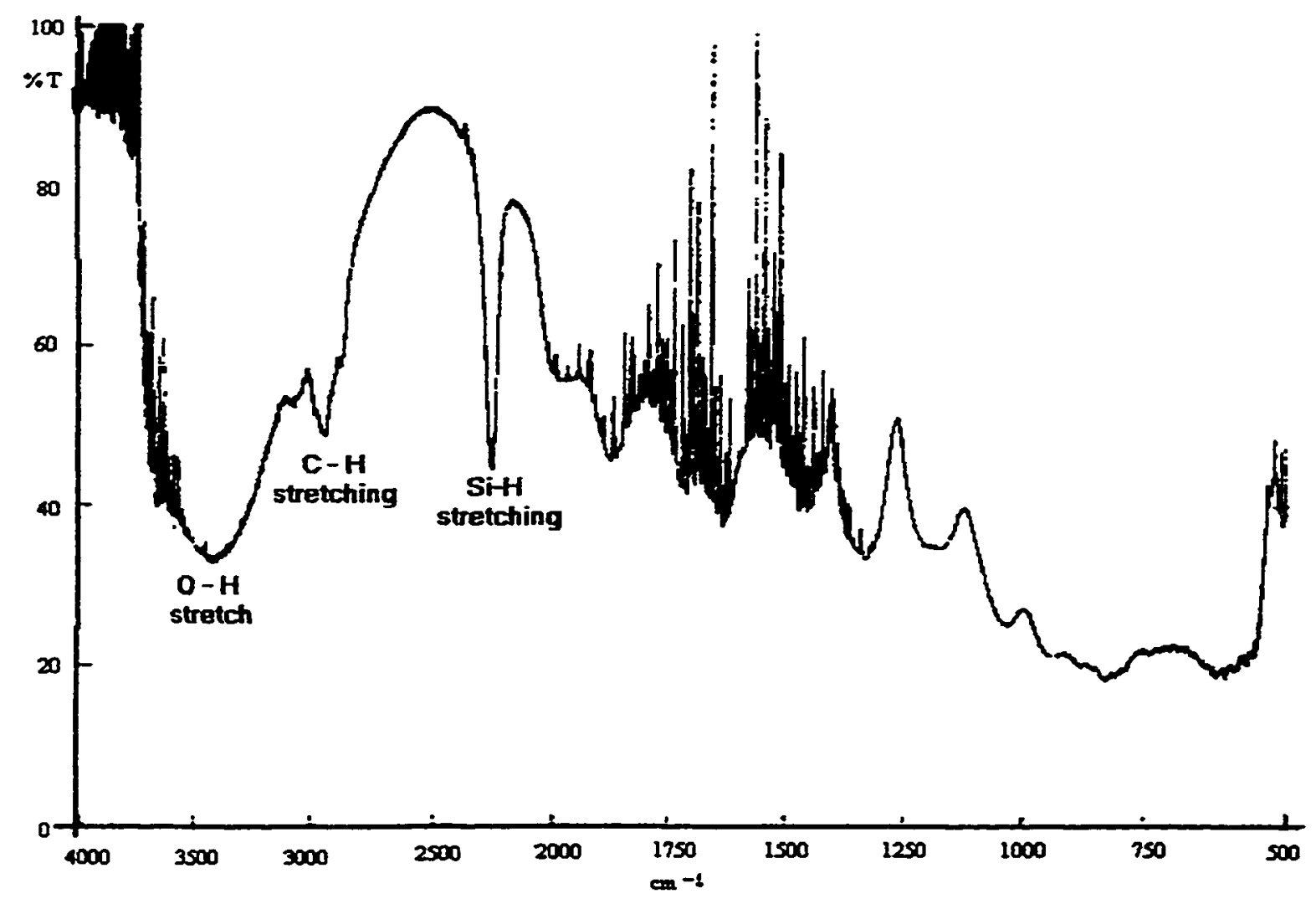

Figure 17. DRIFT spectrum of cholesteryl 10-undecanoate bonded to Vydac silica hydride 


\section{2. ${ }^{13} \mathrm{C}$ CP-MAS NMR of unbonded cholestereyl 10 -undecanoate}

The ${ }^{13} \mathrm{C}$ spectrum of cholesteryl 10 -undecanoate (before bonding) is shown in the Figure 18. The peaks between $112 \mathrm{ppm}$ and $140 \mathrm{ppm}$ are due to the olefinic carbons that are present on the alkyl chains and the olefinic carbons present on the cholesteryl ring. The peaks between 112 and $140 \mathrm{ppm}$ also include the site of hydrosilation. The peaks between 16 to $70 \mathrm{ppm}$ represent the aliphatic carbons of the alkyl chains and also the saturated ring carbons of cholesteryl. The peaks near $172 \mathrm{ppm}$ are due to the carbonyl group [25].

\section{3. ${ }^{13} \mathrm{C}$ CP-MAS NMR of bonded cholestereyl 10 -undecanoate}

Figure 19 shows the ${ }^{13} \mathrm{C}$ CP-MAS NMR spectrum of cholesteryl 10 - undecanoate bonded to silica hydride. There are fewer peaks in the olefinic region as the double bond at the terminal position of one alkyl chain is saturated as a result of hydrosilation. At least one peak is observed in the region around $127 \mathrm{ppm}$. This peak is due to the protonated olefinic carbon in the cholesterol ring structure [25].

D. 1-(2-Cyanoethyl) pyrrole analysis

\section{DRIFT Spectra}

The DRIFT spectrum of the 1-(2-cyanoethyl) pyrrole bonded phase using Wilkinson's catalyst is shown in Figure 20. This figure shows that there is an additional stretching band around $3000 \mathrm{~cm}^{-1}$. This band represents the strong absorbance in the aliphatic $\mathrm{C}-\mathrm{H}$ stretching region indicating the success of bonding. 


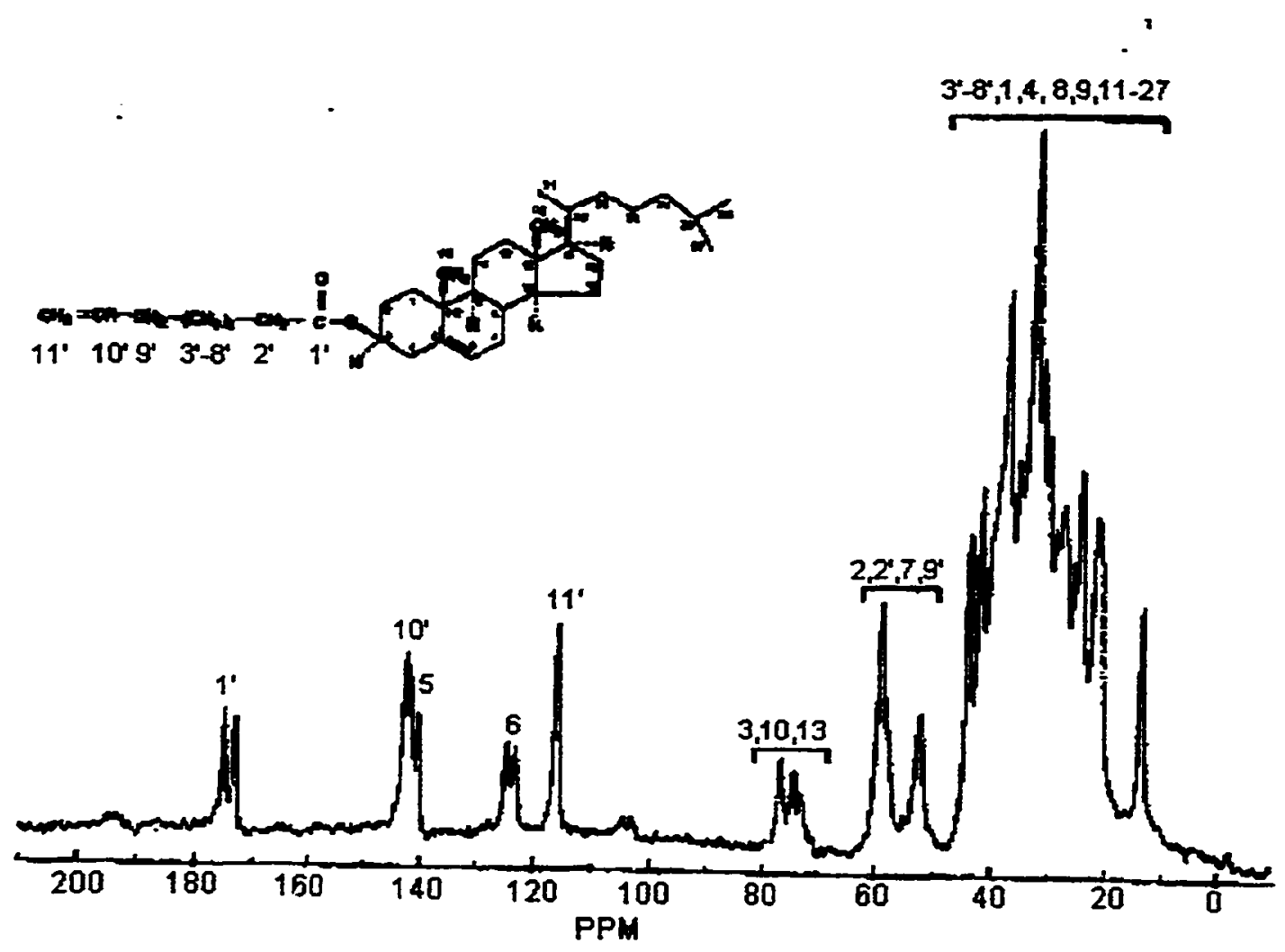

Figure 18. ${ }^{13} \mathrm{C}$ CP-MAS NMR spectrum of cholesteryl 10-undecanoate (before bonding) (taken from reference 25) 


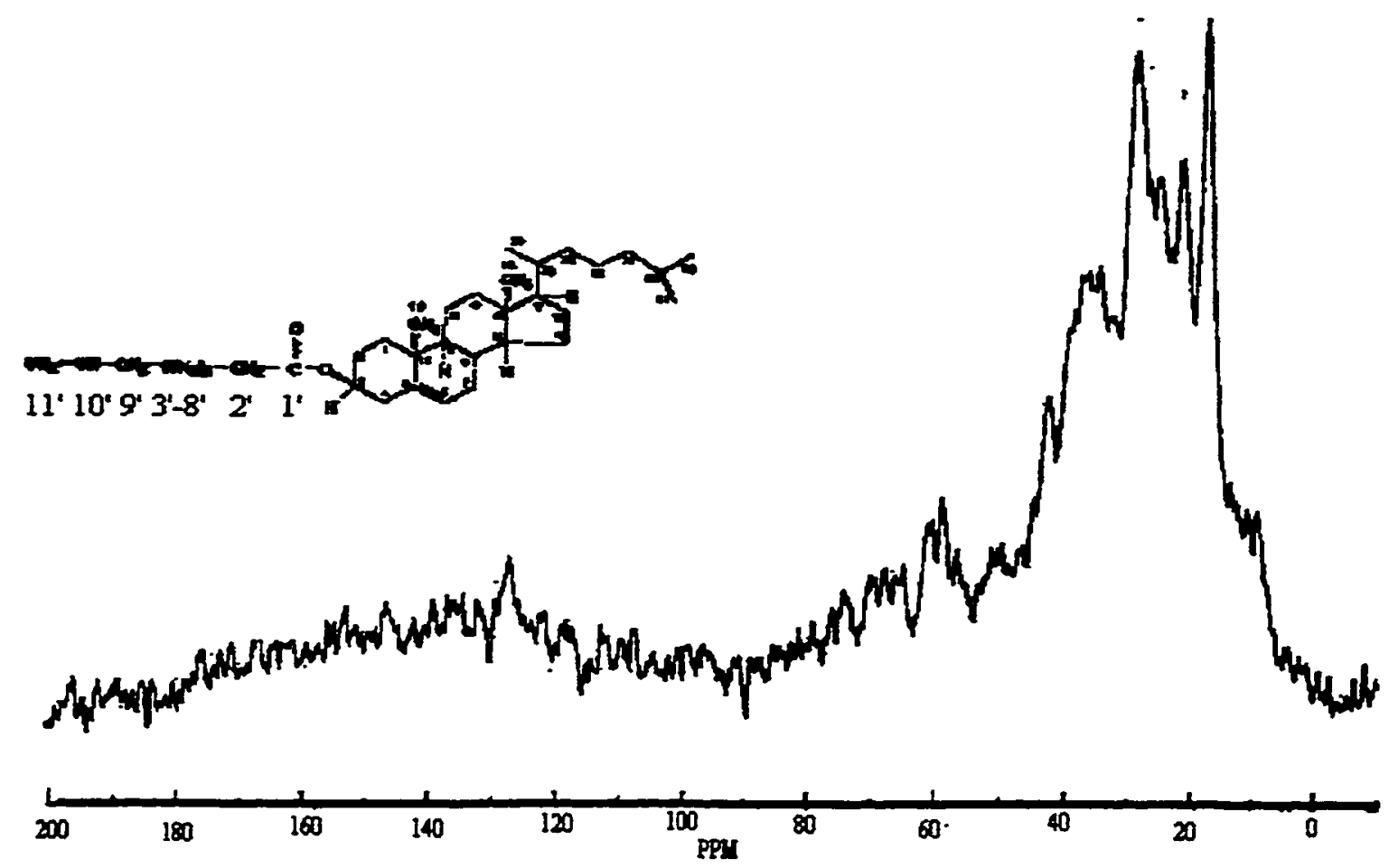

Figure 19. ${ }^{13} \mathrm{C}$ CP-MAS NMR spectrum of cholesteryl 10 - undecanoate bonded to Vydac silica hydride 


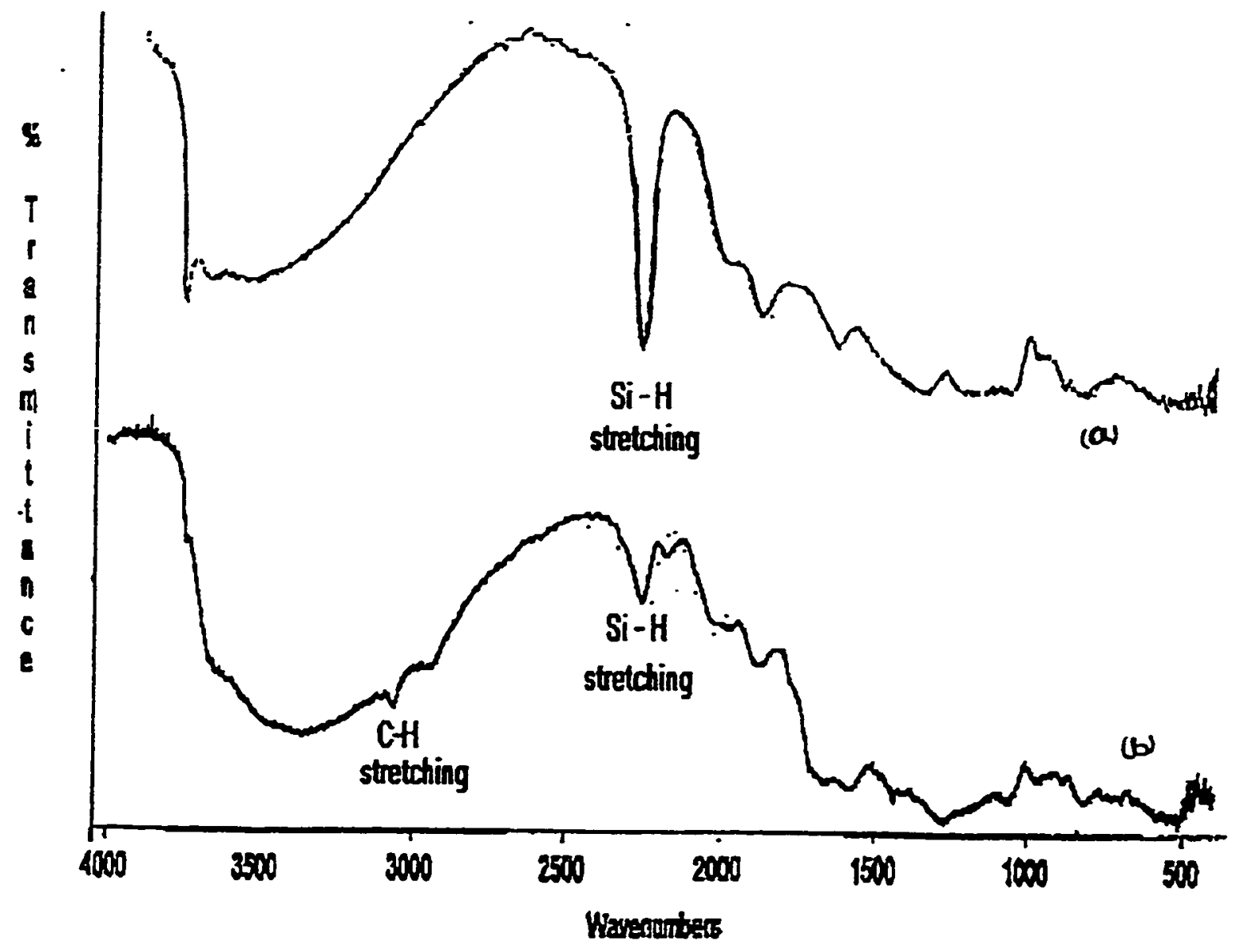

Figure 20. DRIFT Spectrum of 1,2-Cyanoethyl pyrrole bonded to Kromasil silica hydride via Wilkinson's catalyst a)Before hydrosilation b)After Hydrosilation 
The $-\mathrm{CH}_{2} \mathrm{~N}$ group could not be identified as the stretching frequency of this group (between $1600-1750 \mathrm{~cm}^{-1}$ ) is camouflaged by the background signal from the silica matrix. However, this approach for hydrosilation was not used for the synthesis of a bonded stationary phase in this study. The reason for this was the darkening of the material in the bonded phase due to the deposition of the metal on the surface. This effect is probably due to the reduction of the metal in the complex as a result of a reaction between the metal and the surface hydride. Therefore, the free radical initiation method was used for the synthesis of the bonded phase for this study.

The DRIFT spectrum for 1-(2-cyanoethyl) pyrrole bonded phase using AIBN is shown in the Figure 21. This spectrum also shows the strong aliphatic C-H stretching band in the region between $2800 \mathrm{~cm}^{-1}$ and $3000 \mathrm{~cm}^{-1}$. This band confirms the success of bonding. The $-\mathrm{CH}_{2} \mathrm{~N}$ group could not be positively identified because the stretching frequency of this group between $1750 \mathrm{~cm}^{-1}$ and $1600 \mathrm{~cm}^{-1}$ is not evident due to the background signal of the silica matrix.

The DRIFT spectrum of the 1-(2- cyanoethyl) pyrrole bonded phase using tertiary butyl peroxide as the free radical initiator is shown in Figure 22. This spectrum is very similar to the one in Figure 21. The stretching bands between $3000 \mathrm{~cm}^{-1}$ and $2800 \mathrm{~cm}^{-1}$ once again indicate the success of bonding. 


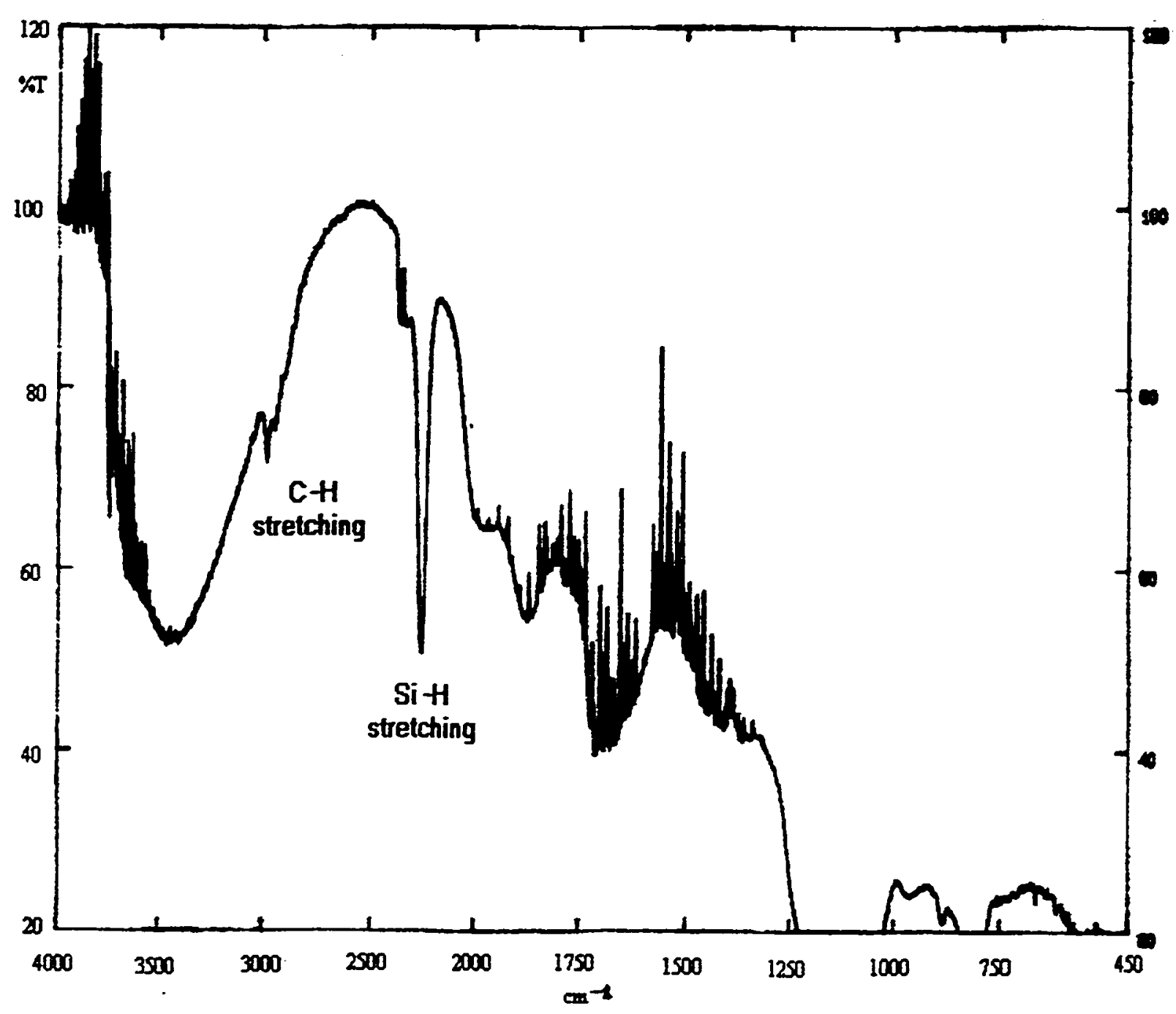

Figure 21. DRIFT Spectrum of 1,2-Cyanoethyl pyrrole bonded to Kromasil hydride via AIBN 


\section{2. ${ }^{29}$ Si CP-MAS NMR}

Figure 23 and Figure 24 represent the ${ }^{29}$ Si CP-MAS NMR spectra for the 1-(2cyanoethyl) pyrrole bonded phase using Wilkinson's catalyst and tertiary butyl peroxide respectively. In these spectra the peak around $101.2 \mathrm{ppm}$ represents silicon atoms that have surface silanols attached to them. The peak at $110.7 \mathrm{ppm}$ is due to the silicon atoms in the siloxane backbone. The peak around $85 \mathrm{ppm}$ indicates the presence of the Si-H group on the surface. However, this peak is considerably smaller than the original peak for silica hydride (before bonding). The reduction in the intensity of this peak also confirms the success of hydrosilation (bonding) process.

\section{3. ${ }^{13} \mathrm{C}$ CP-MAS NMR}

Figure 25 represents the ${ }^{13} \mathrm{C}$ CP-MAS NMR spectrum of $1,(2$-cyanoethyl) pyrrole bonded to Kromasil hydride via the free radical initiator AIBN. In this figure, peaks around 36.0 and $15.4 \mathrm{ppm}$ are due to the alkyl groups. The peaks in the range of $175.4-107.6 \mathrm{ppm}$ are due to the carbons in the pyrrole ring. The peak at $65 \mathrm{ppm}$ could

be due to the $\mathrm{CH}_{2}-\mathrm{N}_{\mathrm{Si}}=$ bonded group. The peak at $84 \mathrm{ppm}$ could be due to an impurity in the sample. This peak could be due to the decomposition product of hydrosilation. However, the exact identity of this peak is unknown as no data is found in the literature on hydrosilation of $1,(2$-cyanoethyl) pyrrole. The peak around $65 \mathrm{ppm}$ in conjunction with other peaks due to pyrrole carbons (175.4 - $107.6 \mathrm{ppm}$ ) and the alkyl groups (36.0 and $15.4 \mathrm{ppm}$ ) confirm the success of bonding. The bonding mechanism is illustrated in the Introduction section. 


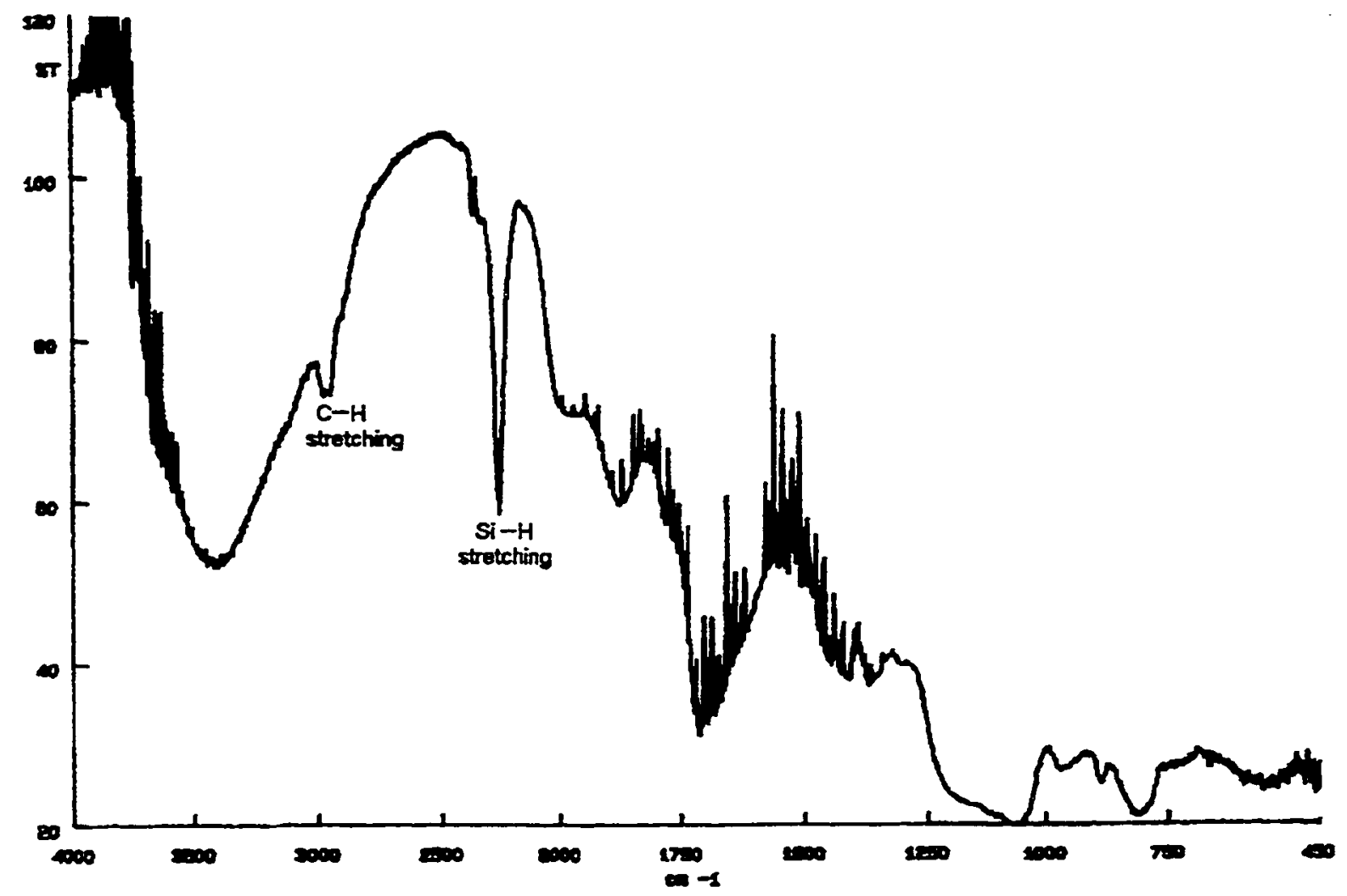

Figure 22. DRIFT Spectrum of 1,2-cyanoethyl pyrrole bonded to Kromasil hydride via $\mathbf{t}$ - butyl peroxide. 


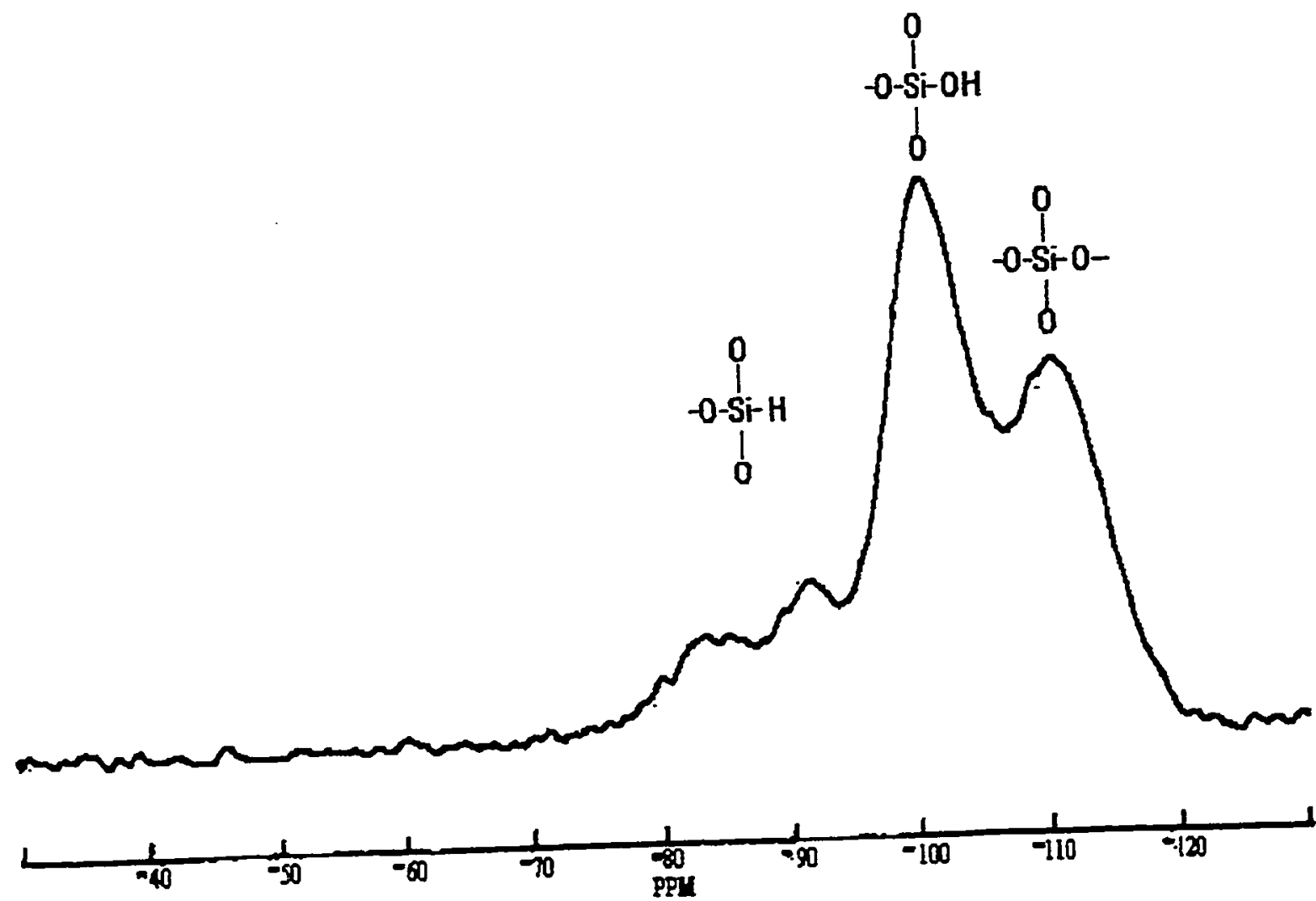

Figure 23. ${ }^{29}$ Si CP-MAS NMR spectrum of 1,2-cyanoethyl pyrrole bonded to Kromasil hydride via Wilkinson's catalyst. 


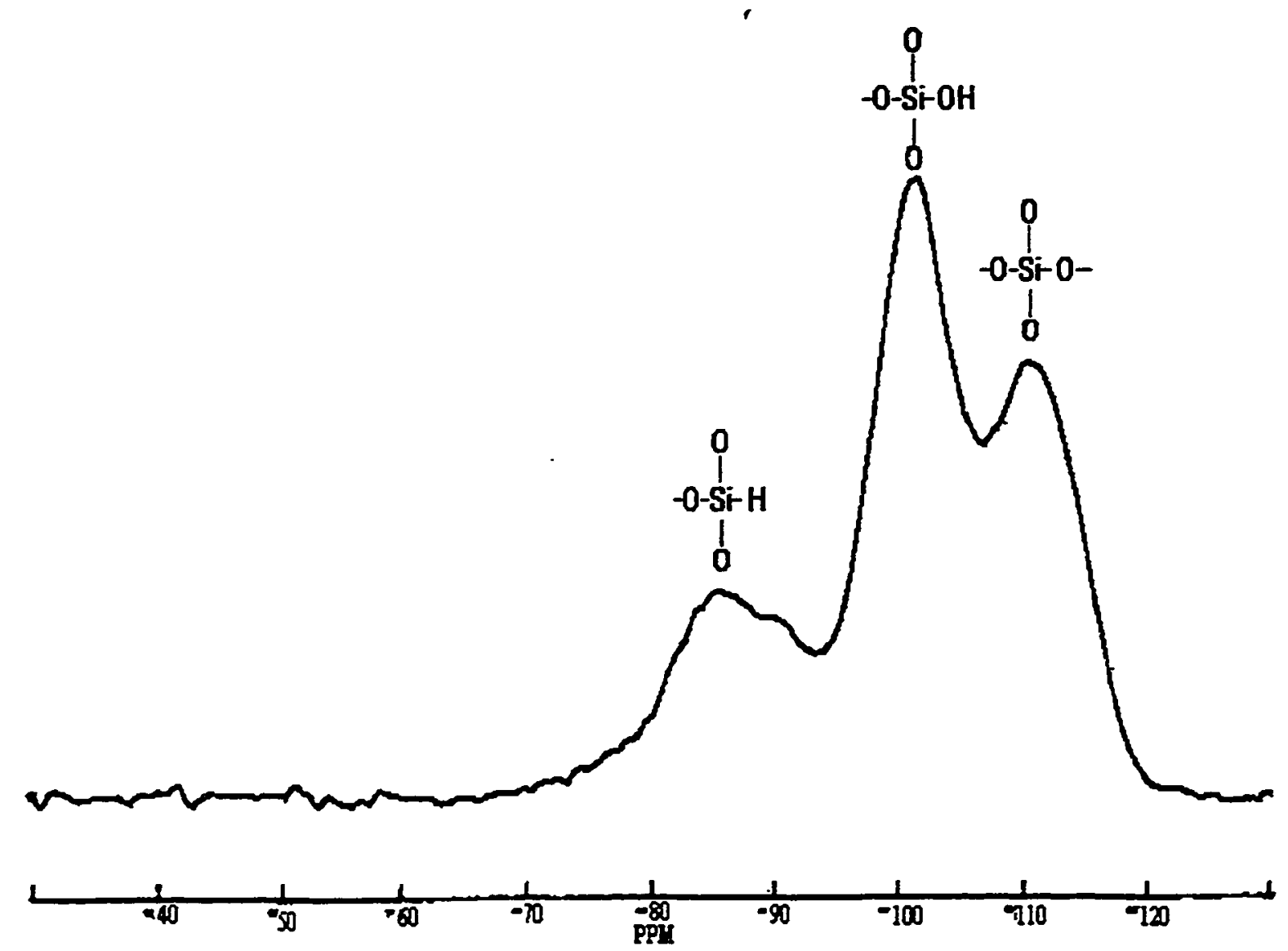

Figure 24. ${ }^{29} \mathrm{Si}$ CP-MAS NMR spectrum of 1,2-cyanoethyl pyrrole bonded to Kromasil hydride via $t$-butyl peroxide 
The ${ }^{13} \mathrm{C}$ CP-MAS NMR spectra of 1, (2 -cyanoethyl) pyrrole bonded to Kromasil hydride via the free radical initiator tertiaty butyl peroxide is shown in Figure 26. In this spectrum, the peaks in the range of $109.2-186.9 \mathrm{ppm}$ are due to the pyrrole carbons. The peaks at $16 \mathrm{ppm}$ and $36.7 \mathrm{ppm}$ are due to the two $-\mathrm{CH}_{2}$ groups. In this spectrum the peak at $65 \mathrm{ppm}$ represents the $\mathrm{CH}_{2}-\mathrm{N} \mathrm{Si}_{\mathrm{Si}}$ bonded group. The peaks due to the pyrrole carbons (109.2 - $186.9 \mathrm{ppm}$ ), alkyl groups (16 ppm and $36.7 \mathrm{ppm}$ ) and the bonding site ( $65 \mathrm{ppm}$ ) confirm the success of bonding. The peak at $83.3 \mathrm{ppm}$ could be an impurity. Figure 27 represents the ${ }^{13} \mathrm{C}$ CP-MAS NMR spectrum of 1, (2-cyanoethyl) pyrrole bonded to Kromasil hydride via Wilkinson's catalyst. This spectrum is a little different from the one obtained using AIBN or t- butyl peroxide. This could be attributed to the fact that these two reactions are following different reaction mechanisms. In this spectrum, the intensity of the peak at $63.5 \mathrm{ppm}$ is greatly increased which might be indicative of the bonding site. The peak at $37.1 \mathrm{ppm}$ represents an alkyl group. However, the presence of only one broad peak (instead of two) in this region indicates that there is some association between the adjacent bonded moieties. The peaks in the range of 107.5 -176.1 are due to the pyrrole carbons. The intensity of the peak at $129.5 \mathrm{ppm}$ is greatly increased. The increase in intensity and shift from $127.8 \mathrm{ppm}$ (for AIBN or t- butyl peroxide) to $129.5 \mathrm{ppm}$ could be due to association between the neighboring bonded groups. This is likely the case because the surface coverage is very high $\left(6.7 \mu \mathrm{mol} / \mathrm{m}^{2}\right)$ as a result of polymerization indicated by broad peaks in the spectrum. 


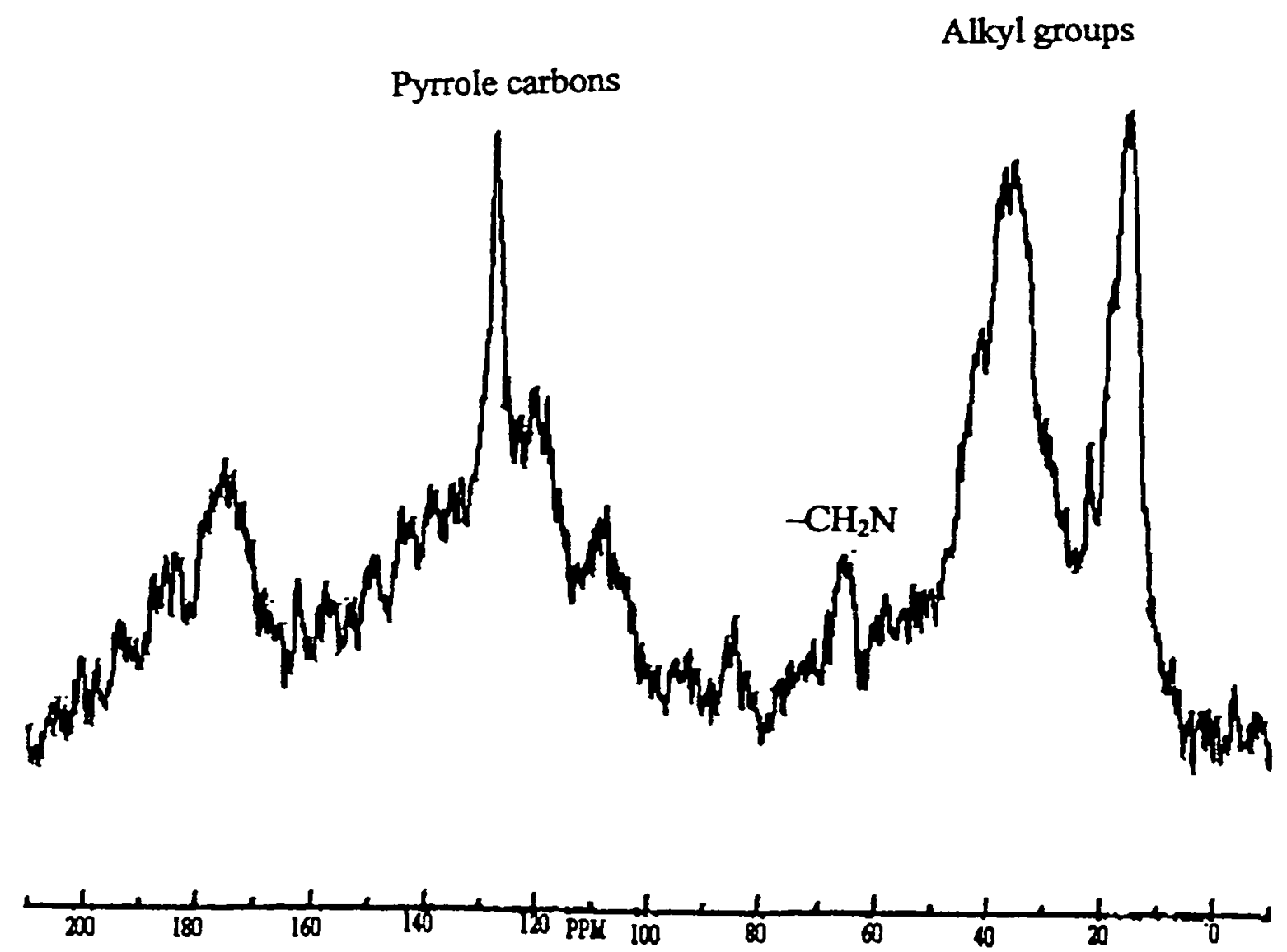

Figure 25. ${ }^{13} \mathrm{C}$ CP-MAS NMR spectrum of 1,2-cyanoethyl pyrrole bonded to Kromasil hydride via AIBN 


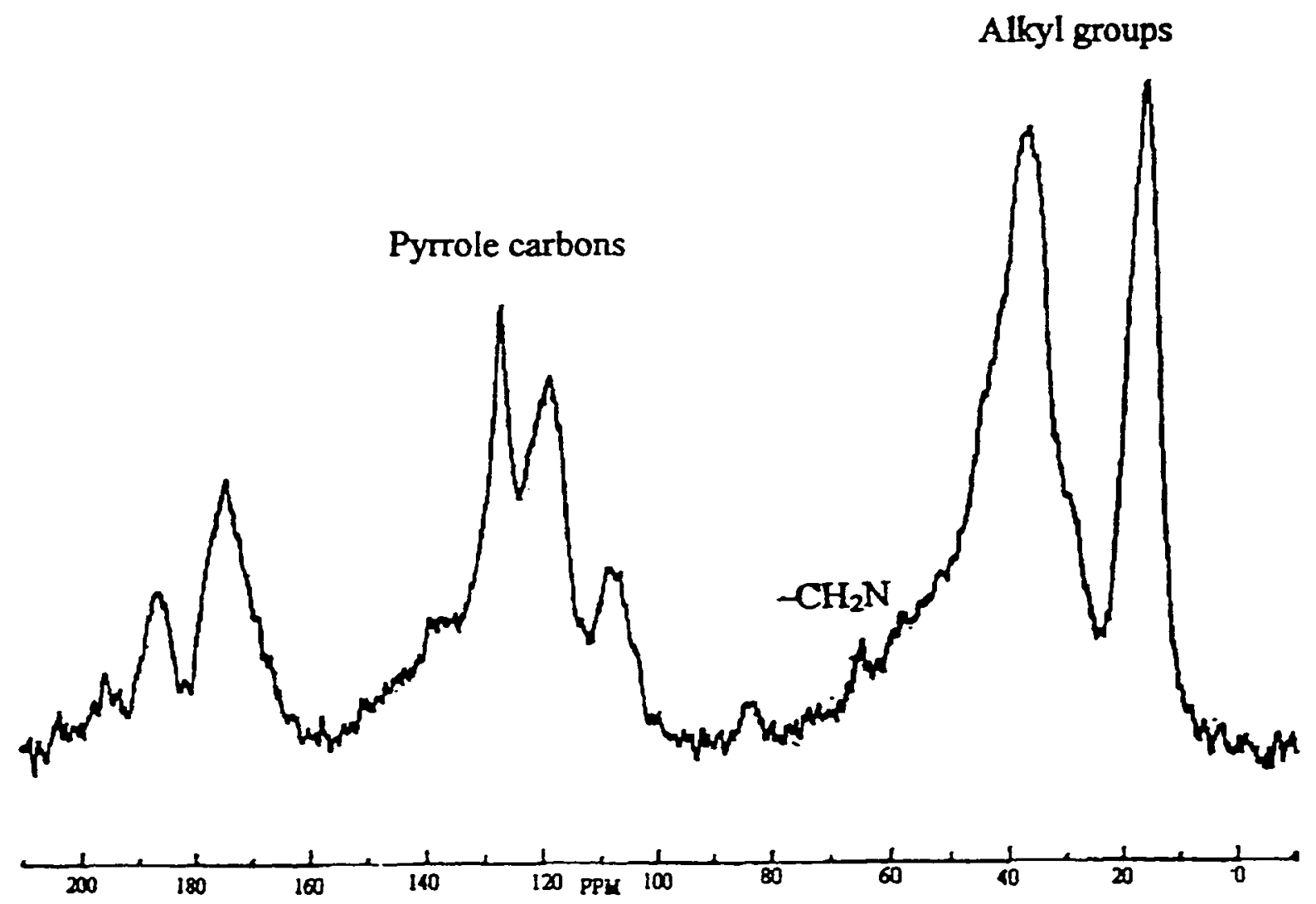

Figure 26. ${ }^{13} \mathrm{C}$ CP-MAS NMR spectrum of 1,2-cyanoethyl pyrrole bonded to Kromasil hydride via $t$-butyl peroxide. 


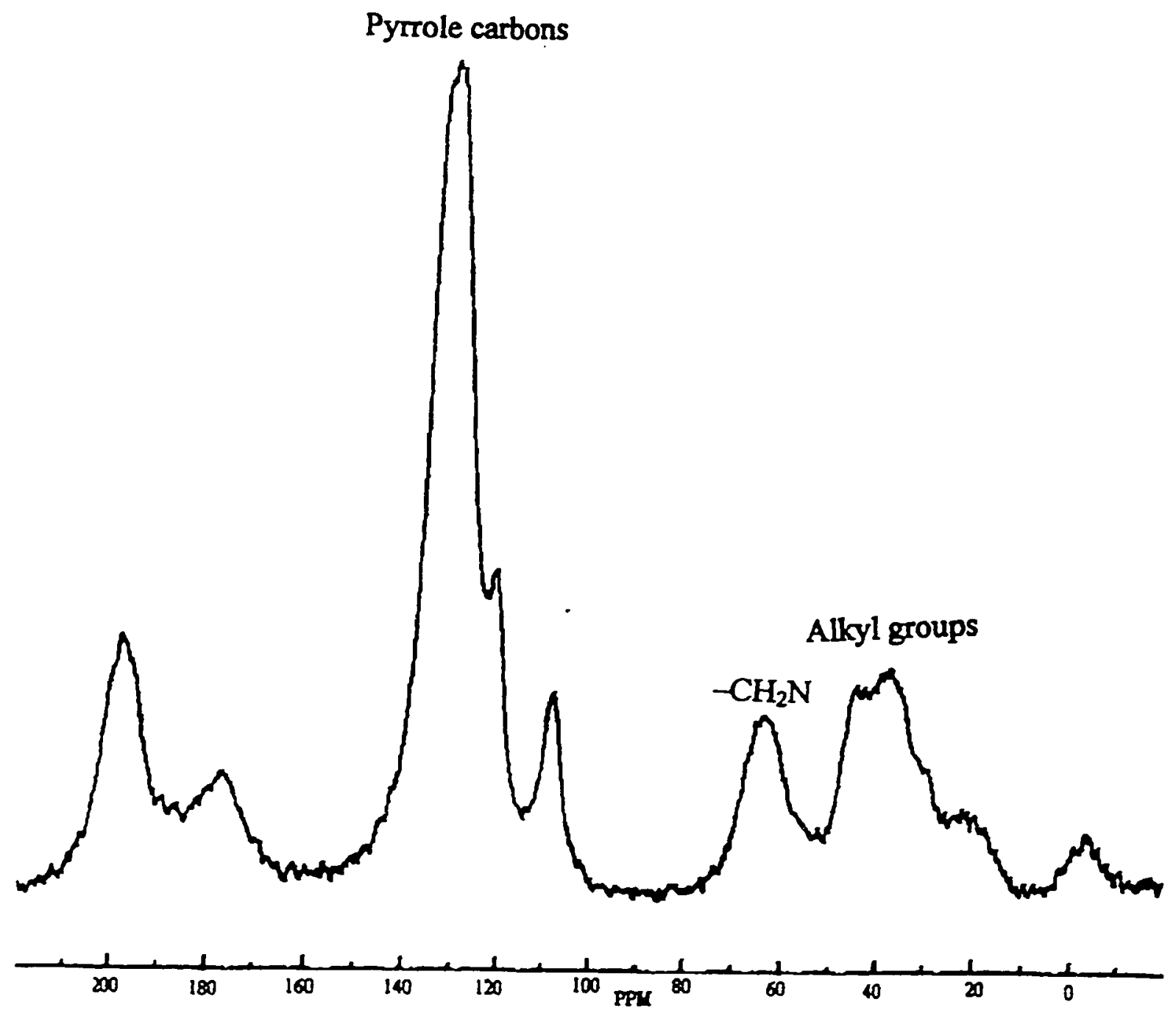

Figure 27. ${ }^{13} \mathrm{C}$ CP-MAS NMR spectrum of 1,2-cyanoethyl pyrrole bonded to Kromasil hydride via Wilkinson's catalyst 


\section{Hydrolytic Stability Test}

\section{a. Acid Hydrolysis DRIFT Spectrum}

Figure 28 represents the DRIFT spectrum of 1,2-cyanoethyl pyrrole bonded to Kromasil silica hydride after acid hydrolysis. All the stretching bands remain unchanged even after the $24 \mathrm{hr}$ acid hydrolysis test. This indicates that the bonded phase is hydrolytically stable under acidic conditions.

\section{b. Base Hydrolysis DRIFT Spectrum}

The DRIFT spectrum of 1,2-cyanoethyl pyrrole bonded to Kromasil hydride after $24 \mathrm{hr}$ base hydrolysis test is shown in the Figure 29. After hydrolysis, stretching bands were identical in position to those obtained before hydrolysis of the bonded phase. However, it appears that $\mathrm{C}-\mathrm{H}$ and $\mathrm{Si}-\mathrm{H}$ bands are reduced in intensity. This confirms the bonded phase is relatively stable and resistant to rapid hydrolysis.

\section{E. 4-cyano-4'-n-pentyl-1,1'-biphenyl analysis}

\section{DRIFT spectrum}

Figure 30 represents the DRIFT spectrum of 4-cyano-4'-n-pentyl-1,1'-biphenyl bonded to Kromasil silica hydride via the free radical initiator tertiary butyl peroxide. In this spectrum, the stretching band at $2250 \mathrm{~cm}^{-1}$ is due to the $\mathrm{Si}-\mathrm{H}$ bond. The stretching bands between $2800-3000 \mathrm{~cm}^{-1}$ are due to aliphatic $\mathrm{C}-\mathrm{H}$ bonds. The weaker bands near $3100 \mathrm{~cm}^{-1}$ are due to the aromatic $\mathrm{C}-\mathrm{H}$ bonds. These two features confirm that the $\mathrm{Si}-\mathrm{H}$ group has undergone chemical reaction. 


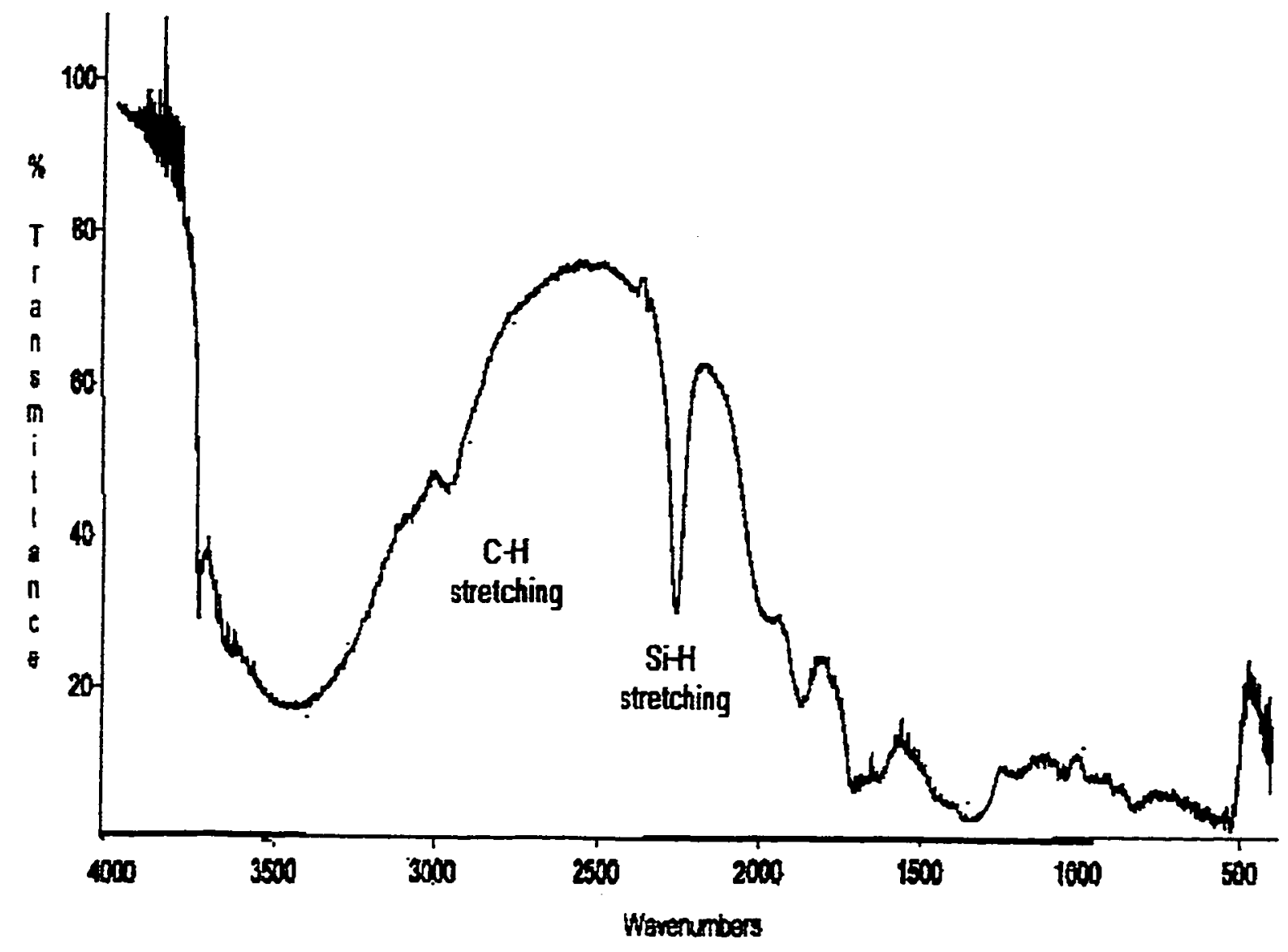

Figure 28. DRIFT spectrum of 1,2 cyanoethyl pyrrole bonded to Kromasil hydride after acid hydrolysis 


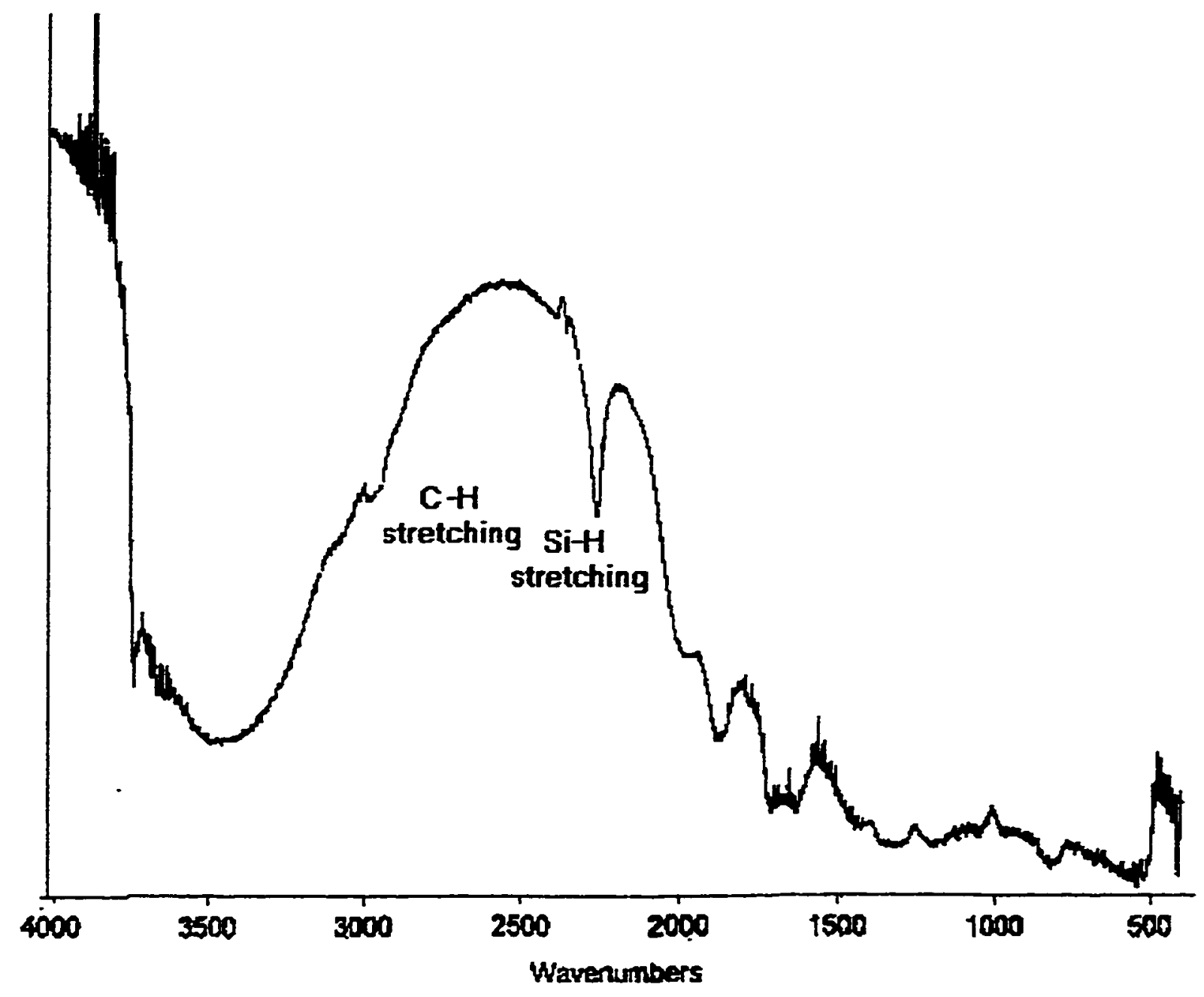

Figure 29. DRIFT spectrum of 1,2 cyanoethyl pyrrole bonded to Kromasil hydride after base hydrolysis 


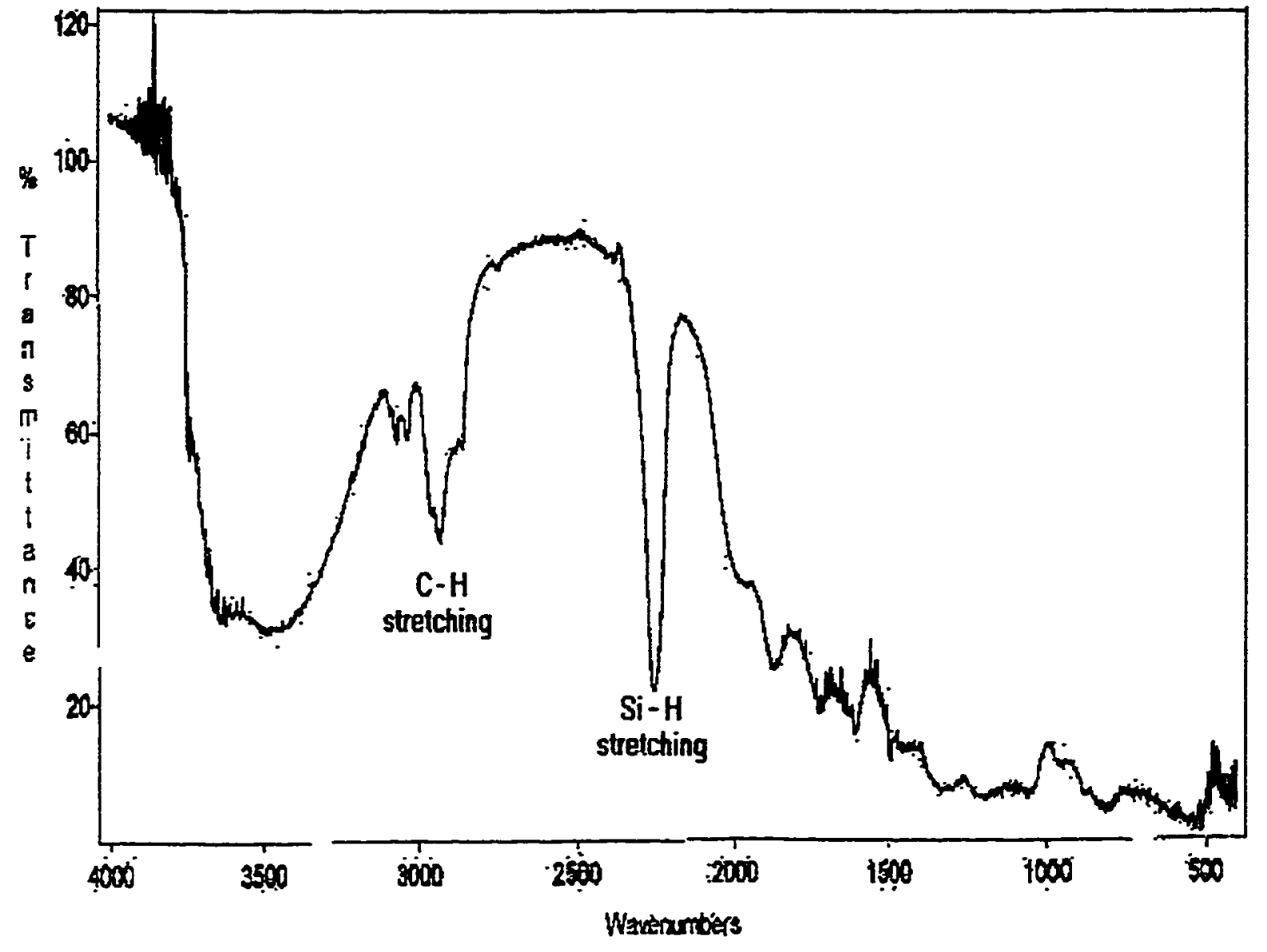

Figure 30. DRIFT spectrum of 4-cyano-4'-n-pentyl-1,1'-biphenyl bonded to Kromasil silica hydride via free radical initiator tertiary butyl peroxide 
However, the appearance of a band in the stretching frequency for $-\mathrm{CH}_{2} \mathrm{~N}-$ that occurs between $1600 \mathrm{~cm}^{-1}$ and $1750 \mathrm{~cm}^{-1}$ is camouflaged due to the presence of very intense $-\mathrm{Si}-\mathrm{O}$-absorbance from the silica.

Figure 31 represents the DRIFT spectrum of 4-cyano-4'-n-pentyl-1,1'-biphenyl bonded to Vydac silica hydride via tertiary butyl peroxide. This spectrum is similar to Figure 30 except that the intensity of all the stretching bands is slightly reduced. This could be due to the fact that Kromasil silica has a surface area of $340 \mathrm{~m}^{2} / \mathrm{g}$, which is three times larger than that of Vydac silica $\left(106 \mathrm{~m}^{2} / \mathrm{g}\right)$. This increase in surface area is directly proportional to the number of bonding groups on the surface and thus the intensity of the absorption bands.

\section{Hydrolytic Stability Test}

\section{a. Acid Hydrolysis DRIFT Spectrum}

Figure 32 represents the DRIFT spectrum of 4-cyano-4'-n-pentyl-1,1 '-biphenyl bonded to Kromasil silica hydride via tertiary butyl peroxide. All the stretching bands remain unchanged in position after the $24 \mathrm{hr}$ acid hydrolysis test but are lower in intensity. This shows that the bonded phase has reasonable hydrolytic stability under acidic conditions.

\section{b. Base Hydrolysis DRIFT Spectrum}

The DRIFT spectrum of 4-cyano-4'-n-pentyl-1,1'-biphenyl bonded to Kromasil hydride after base hydrolysis is shown in the Figure 33 . This spectrum also shows slightly reduced intensity of the $\mathrm{C}-\mathrm{H}$ bands which indicates that this bonded phase has reasonable stability under basic conditions. 


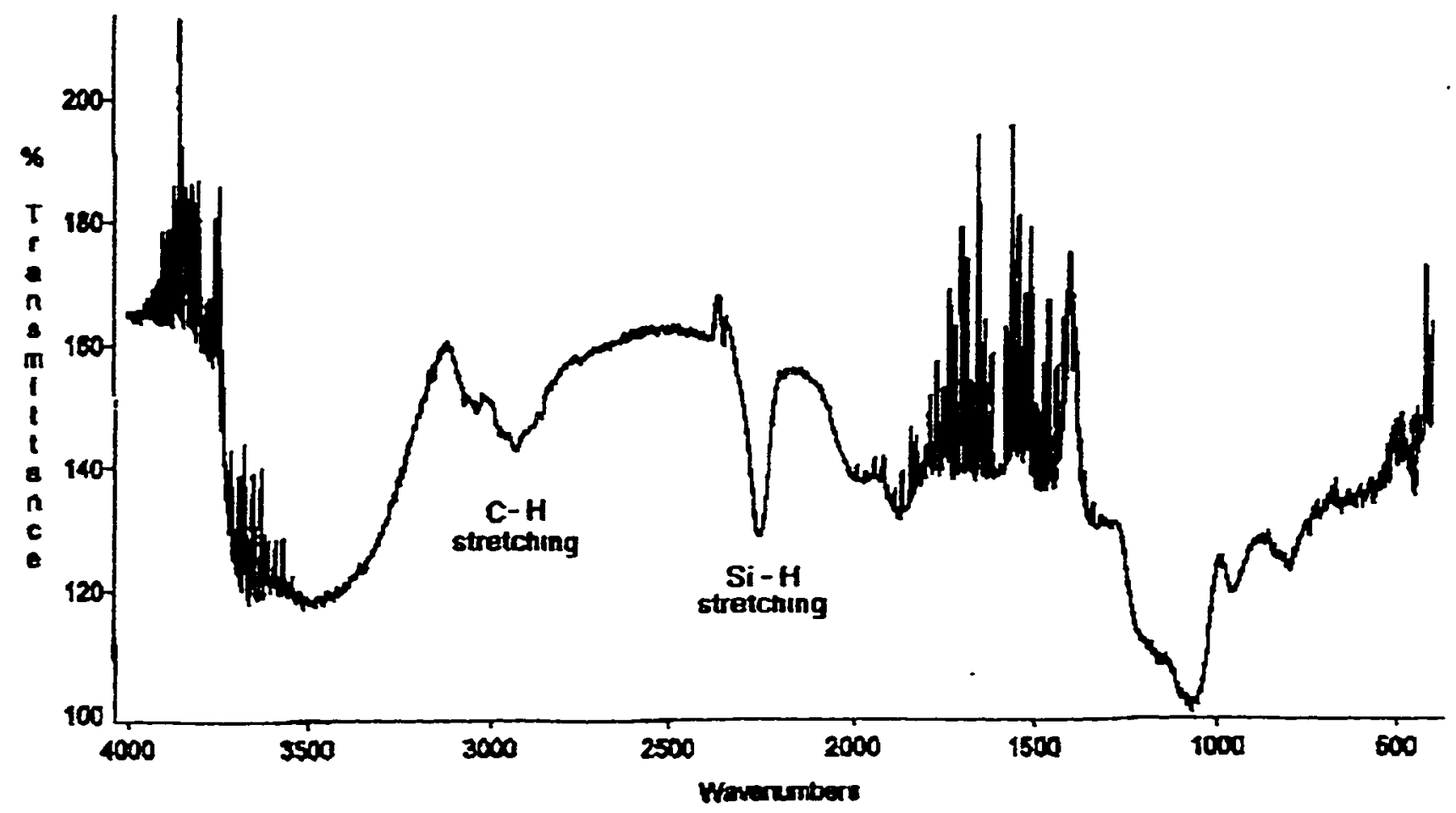

Figure 31. DRIFT spectrum of 4-cyano-4'-n-pentyl-1,1'-biphenyl bonded to Vydac silica hydride via free radical initiator tertiary butyl peroxide 


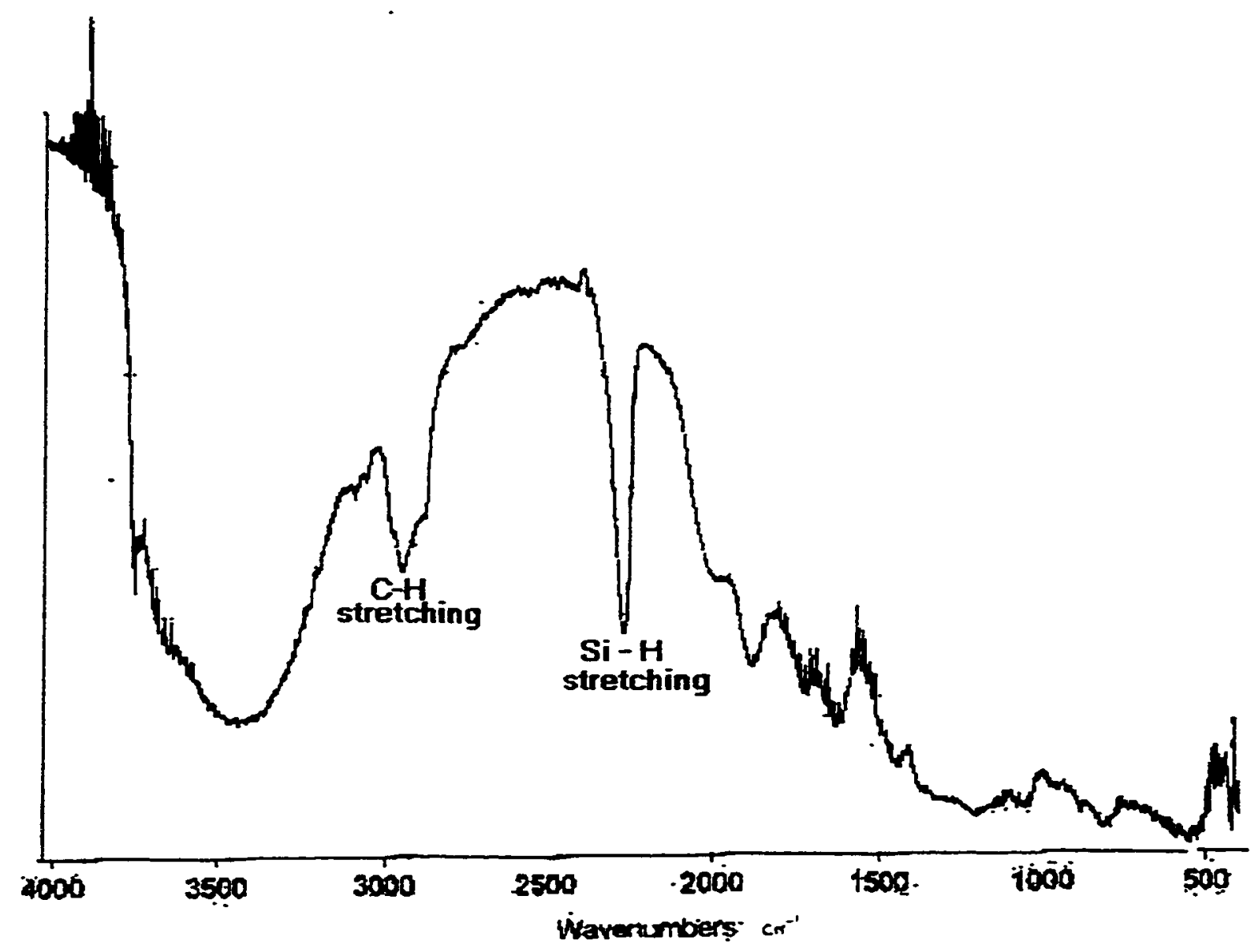

Figure 32. DRIFT spectrum of 4-cyano-4'-n-pentyl-1,1'-biphenyI bonded to Kromasil hydride after acid hydrolysis 


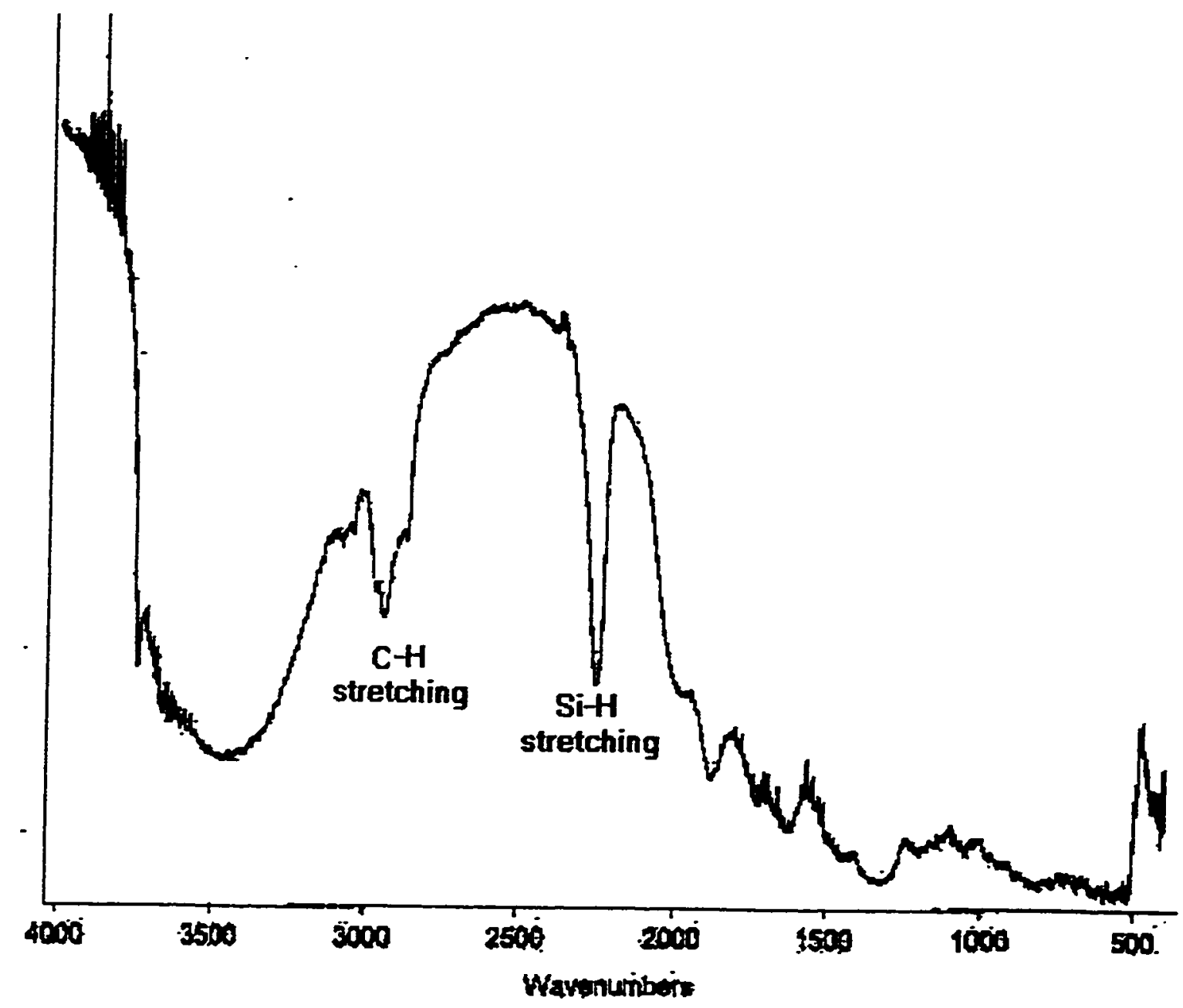

Figure 33. DRIFT spectrum of 4-cyano-4'-n-pentyl-1,1'-biphenyl bonded to Kromasil hydride after base hydrolysis. 


\section{3. ${ }^{29} \mathrm{Si}$ CP-MAS NMR}

Figure 34 represents the ${ }^{29}$ Si CP-MAS NMR spectrum of 4-cyano-4 - -n-pentyl1,1'-biphenyl bonded to Kromasil hydride. In this figure, the $\mathrm{Si}-\mathrm{H}$ peak around -84.4 $\mathrm{ppm}$ is the result of silanization process. The peak at $-102.4 \mathrm{ppm}$ is due to the silicon atom with three siloxane linkages and one hydroxyl group. The peak at $-110.4 \mathrm{ppm}$ is due to the siloxane backbone. The intensity of the peak at $-84.4 \mathrm{ppm}$ is diminished indicating the success of hydrosilation process.

\section{4. ${ }^{13} \mathrm{C}$ CP-MAS NMR}

Figure 35 represents the ${ }^{13} \mathrm{C}$ CP-MAS NMR spectrum of 4-cyano-4' -n-pentyl1,1 -biphenyl bonded to Kromasil hydride at $50^{\circ} \mathrm{C}$. In this spectrum the peaks in the range of $28 \mathrm{ppm}$ to $72 \mathrm{ppm}$ are due to the aliphatic carbons present. The peaks are broad which could be due to a high degree of association between the neighboring bonded groups or the relaxation of the carbons in this region of the bonded group is very fast. The peaks in the range of $120 \mathrm{ppm}$ to $160 \mathrm{ppm}$ represent the aromatic carbons in the bonded group. The peak at $138.6 \mathrm{ppm}$ is due to the unprotonated aromatic carbons and the peak at $127 \mathrm{ppm}$ is due to closely associated aromatic carbons indicating good proton crosspolarization because of its high intensity. The peak at $65 \mathrm{ppm}$ represents the bonded group. This peak along with the other peaks due to aromatic and aliphatic carbons of the bonded group confirm the success of bonding. 


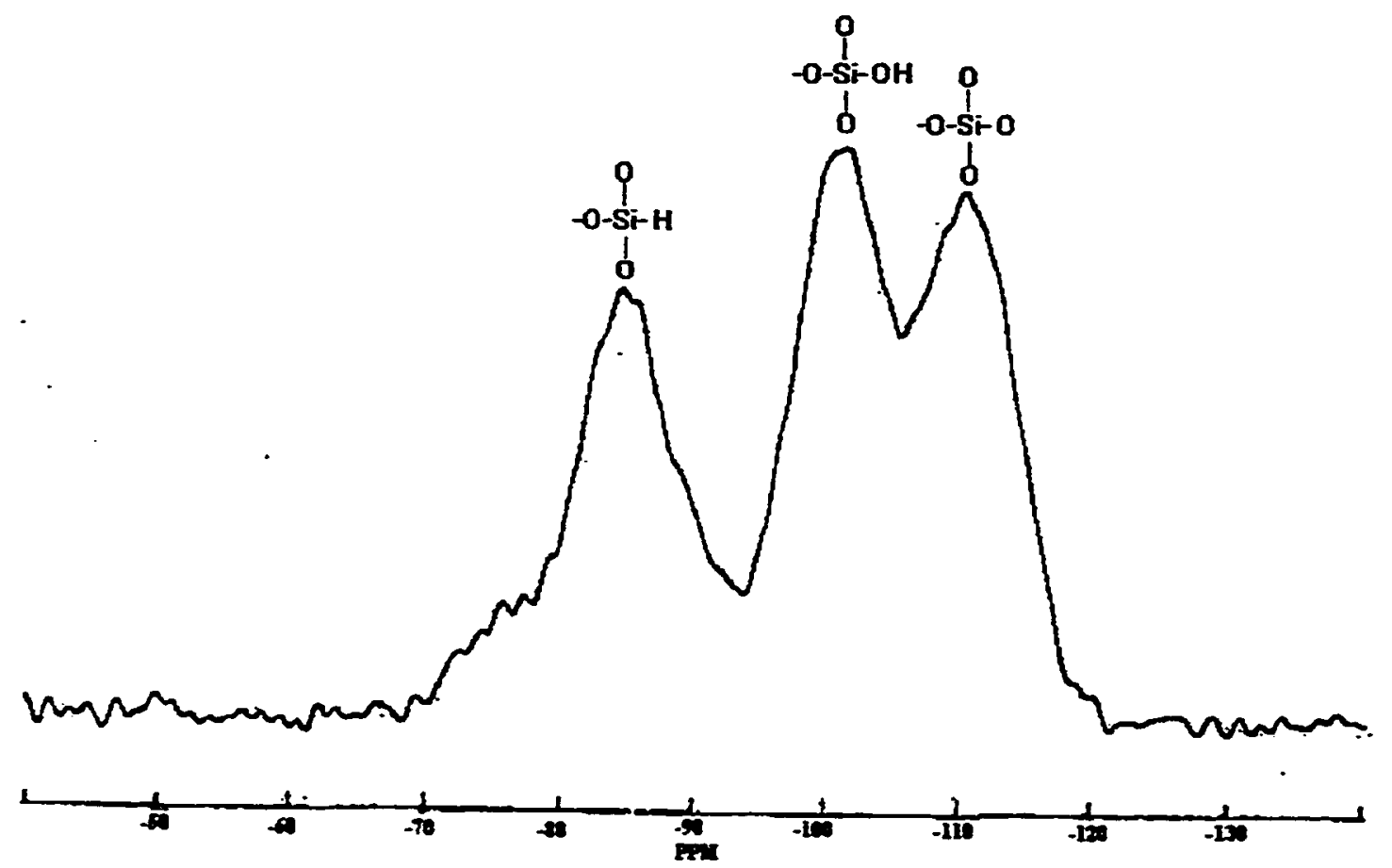

Figure 34. ${ }^{29} \mathrm{Si}$ CP-MAS NMR spectrum of 4-cyano-4'-n-pentyl-1, $1^{\prime}$-biphenyl bonded to Kromasil hydride 


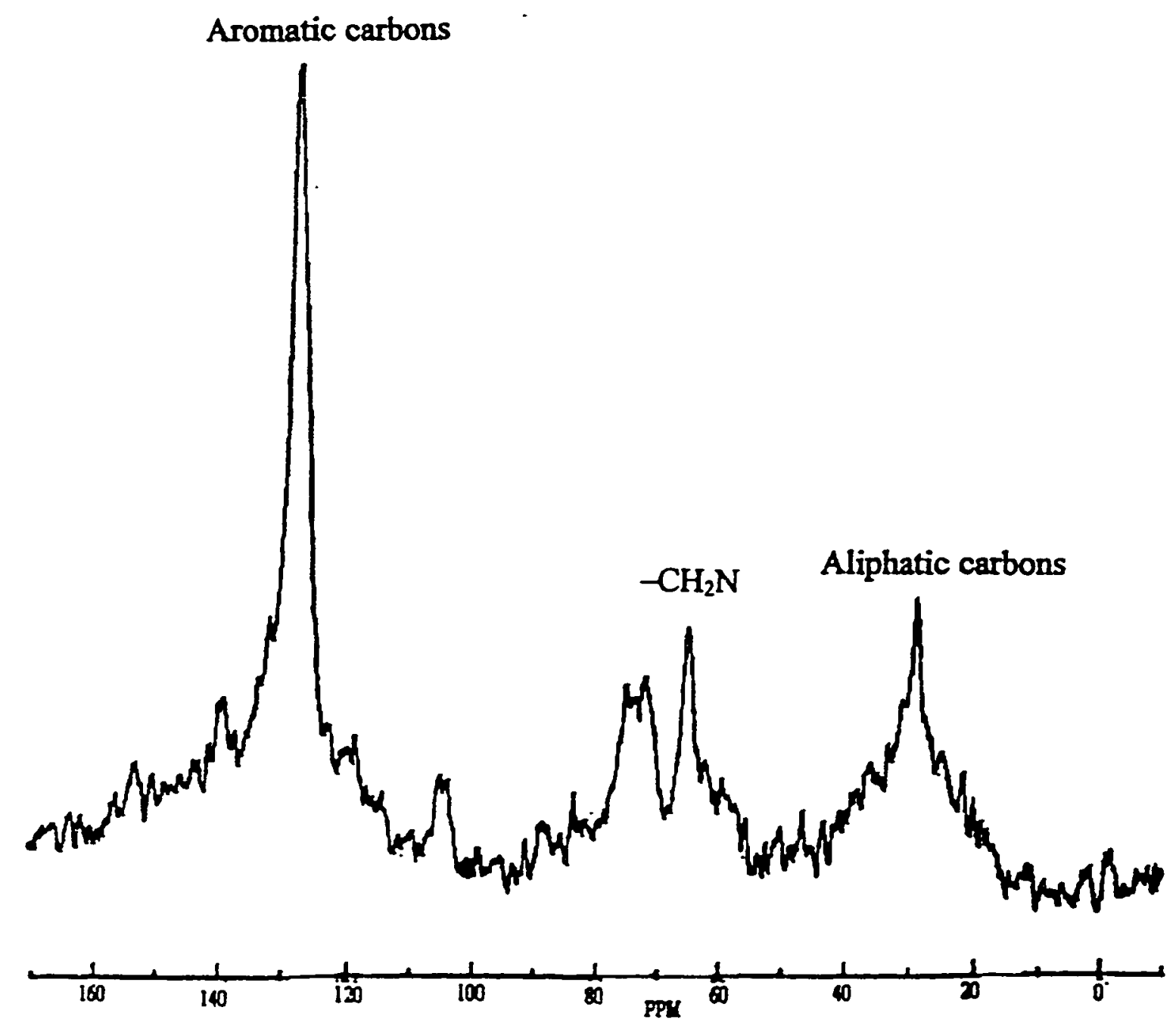

Figure 35. ${ }^{13} \mathrm{C}$ CP-MAS NMR spectrum of 4-cyano-4'-n-pentyl-1, $1^{\prime}$-biphenyl bonded to Kromasil hydride at $50^{\circ} \mathrm{C}$ 


\section{5. ${ }^{13} \mathrm{C}$ CP-MAS NMR}

The ${ }^{13} \mathrm{C}$ CP-MAS NMR spectrum of 4-cyano-4'-n-pentyl-1, $1^{\prime}$-biphenyl bonded to Kromasil hydride at $25^{\circ} \mathrm{C}$ is shown in Figure 36. This spectrum is identical to the one obtained at $50^{\circ} \mathrm{C}$ except that the intensity of some of the peaks is reduced and some of the peaks are slightly broad (peaks around $28 \mathrm{ppm}, 71 \mathrm{ppm}$ and $104 \mathrm{ppm}$ ). At lower temperatures many peaks broaden to such an extent that they completely disappear. Highly restricted motion giving rise to the changes in $T_{1}$ (spin-spin relaxation time) could account for this observation. The peak at $138.6 \mathrm{ppm}$ represents the unprotonated aromatic carbons. The peaks in the range of $104 \mathrm{ppm}$ to $127.2 \mathrm{ppm}$ are due to the protonated aromatic carbons indicating efficient proton transfer. The peak at 71.6 is probably due to some impurity. The peak at $65 \mathrm{ppm}$ is the result of bonding indicating the presence of the

$\mathrm{CH}_{2}-\mathrm{N}_{\mathrm{Si}_{i}} \mathrm{Si}_{-}$group. The presence of this feature in conjunction with the other peaks due to aliphatic and aromatic carbons indicate the success of bonding.

\section{F. 4-cyano-4'-n-pentoxy-1,1'-biphenyl analysis}

\section{DRIFT Spectra}

Figure 37 represents the DRIFT spectrum of 4-cyano-4'-n-pentoxy-1,1'-biphenyl bonded to Kromasil silica hydride via the free radical initiator tertiary butyl peroxide. In this spectrum, the stretching band at $2250 \mathrm{~cm}^{-1}$ is due to the Si-H bond. The stretching band due to $-\mathrm{CH}_{2} \mathrm{~N}$ - that occurs between $1600 \mathrm{~cm}^{-1}$ and $1750 \mathrm{~cm}^{-1}$ is not evident because of the presence of background signal from the silica matrix. The stretching bands between $2800-3000 \mathrm{~cm}^{-1}$ are due to aliphatic C-H bonds. The bands between $3000-$ 
$3100 \mathrm{~cm}^{-1}$ are due to the aromatic $\mathrm{C}-\mathrm{H}$ bonds. These two features confirm that the $\mathrm{Si}-\mathrm{H}$ group has undergone chemical reaction.

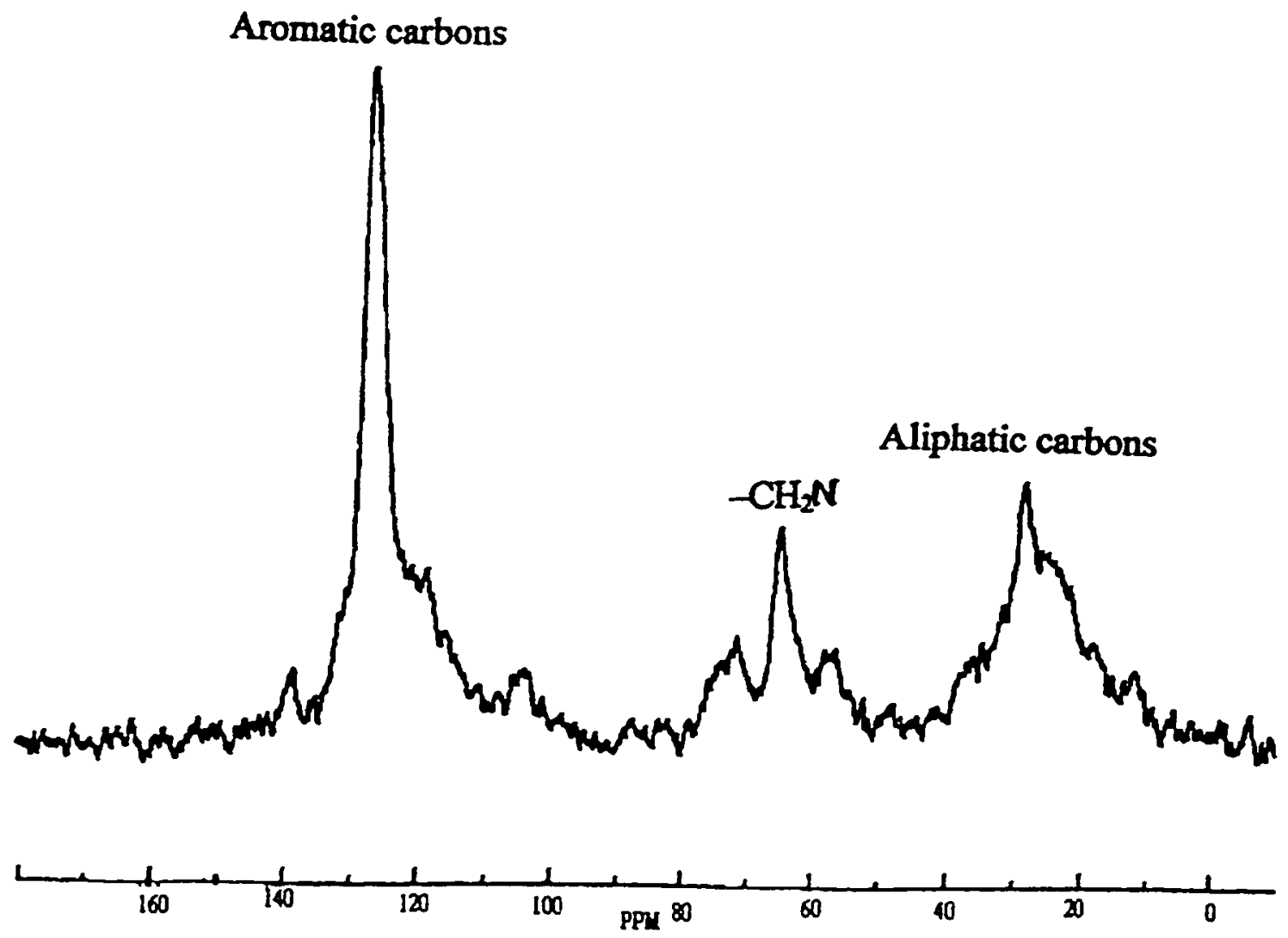

Figure 36. ${ }^{13} \mathrm{C}$ CP-MAS NMR spectrum of 4-cyano-4 -n-pentyl-1,1 -biphenyl bonded to Kromasil hydride $\left(25^{\circ} \mathrm{C}\right)$ 
Figure 38 represents the DRIFT spectrum of 4 - cyano-4'-n-pentoxy $-1,1$ '-biphenyl bonded to Vydac silica hydride via tertiary butyl peroxide. This spectrum is similar to Figure 37 except that the intensity of all the stretching bands is slightly reduced. This could be attributed to the differences in the surface area of Kromasil and Vydac silicas. Kromasil silica $\left(340 \mathrm{~m}^{2} / \mathrm{g}\right)$ has a larger surface area that results in large number of bonded groups at the surface. In contrast, Vydac $\left(106 \mathrm{~m}^{2} / \mathrm{g}\right)$ silica has a surface area which is nearly three times smaller than the Kromasil silica, resulting in smaller number of bonded groups at the surface. This lower surface area can be observed as a decrease in the intensity of the stretching bands.

\section{2. ${ }^{29}$ Si CP-MAS NMR}

Figure 39 represents the ${ }^{29} \mathrm{Si}$ CP-MAS NMR spectrum of 4-cyano-4'-n-pentoxy $1,1^{1}$-biphenyl bonded to Kromasil hydride. In this figure, the peak at $-110.4 \mathrm{ppm}$ is due to the siloxane backbone. The peak at $-102.4 \mathrm{ppm}$ is represents a silicon atom with three siloxane linkages and one hydroxyl group. The peak near $-84.4 \mathrm{ppm}$ represents the $\mathrm{Si}-\mathrm{H}$ group indicating the result of silanization process. The intensity of this peak is reduced after bonding. This result shows that the hydrosilation process was complete and that the liquid crystal moiety has been successfully bonded to the silica hydride. 


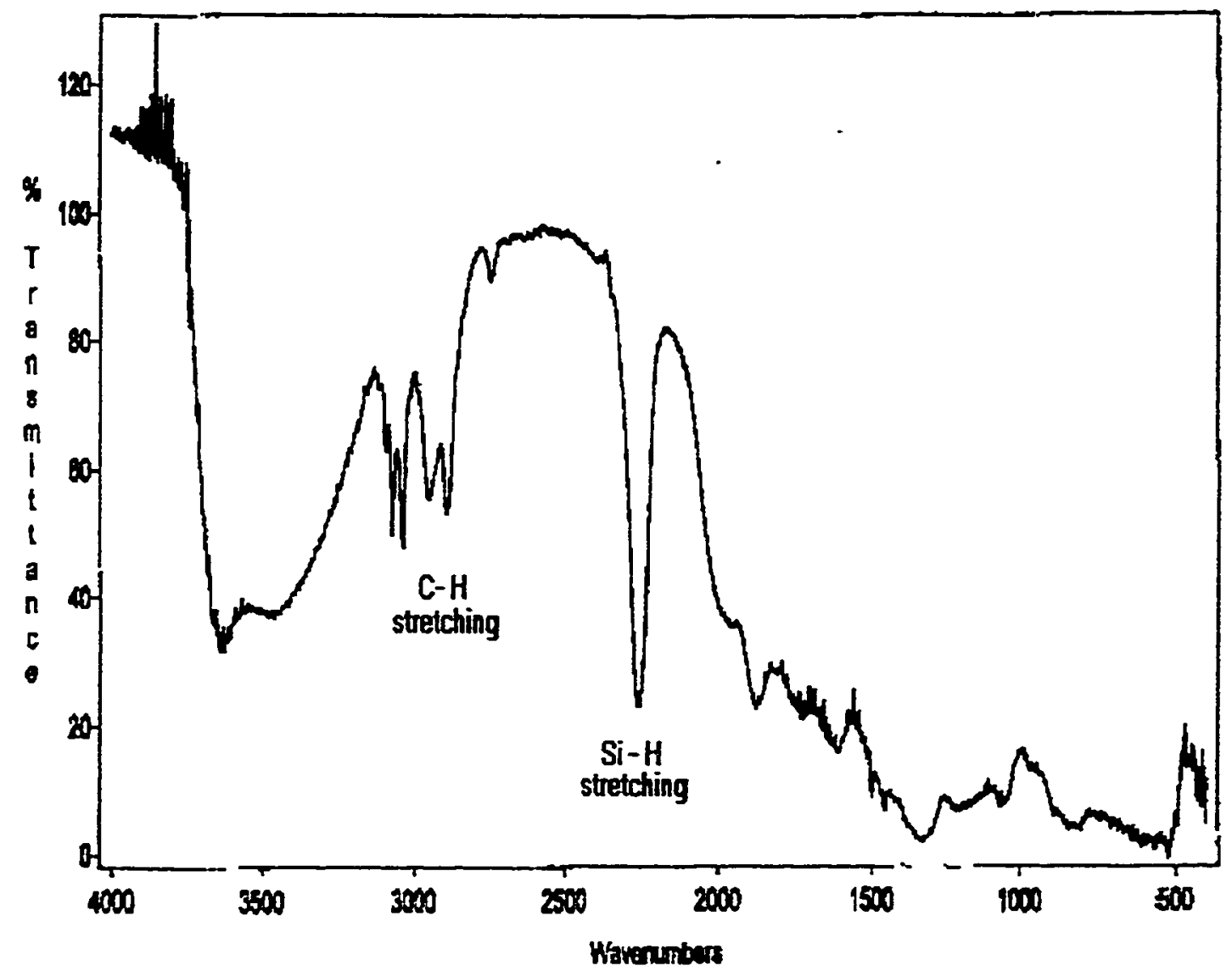

Figure 37. DRIFT spectrum of 4-cyano-4'-n-pentoxy-1,1'-biphenyl bonded to Kromasil silica hydride via free radical initiator tertiary butyl peroxide 


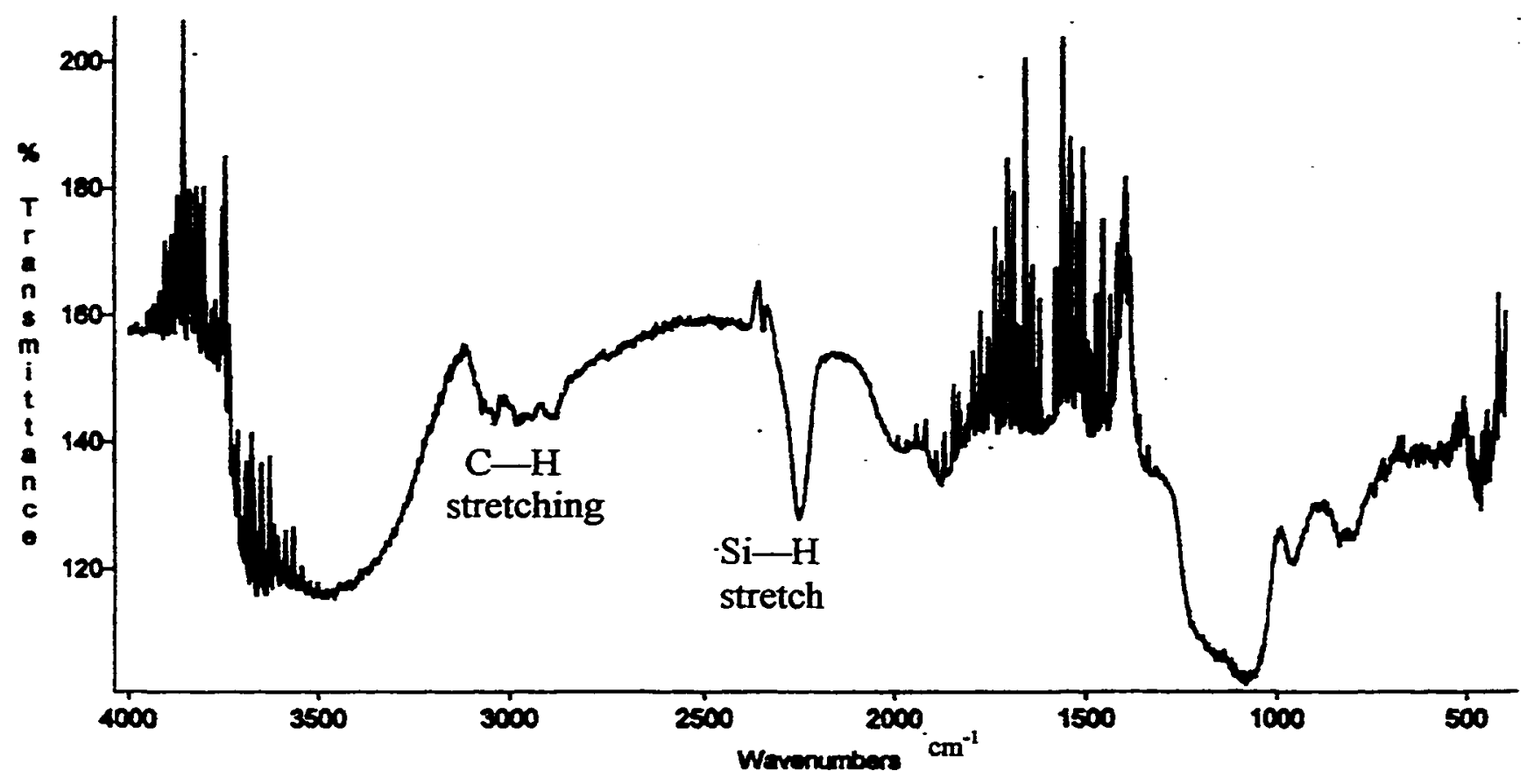

Figure 38. DRIFT spectrum of 4 - cyano-4'-n-pentoxy $-1,1^{\prime}$-bihenyl bonded to Vydac silica hydride via tertiary butyl peroxide 


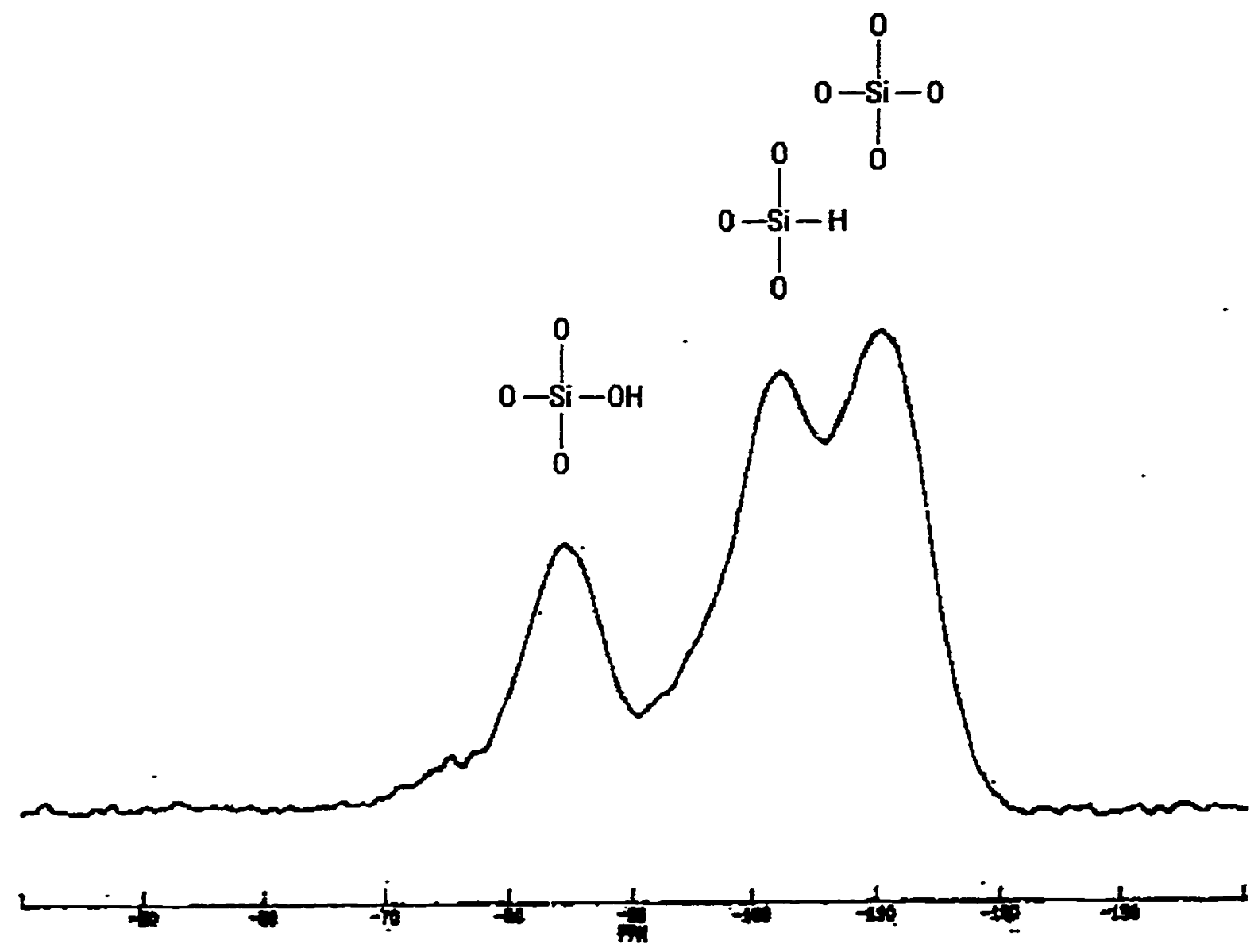

Figure 39. ${ }^{29}$ Si CP-MAS NMR spectrum of 4-cyano-4'-n-pentoxy -1,1'-biphenyl bonded to Kromasil hydride 


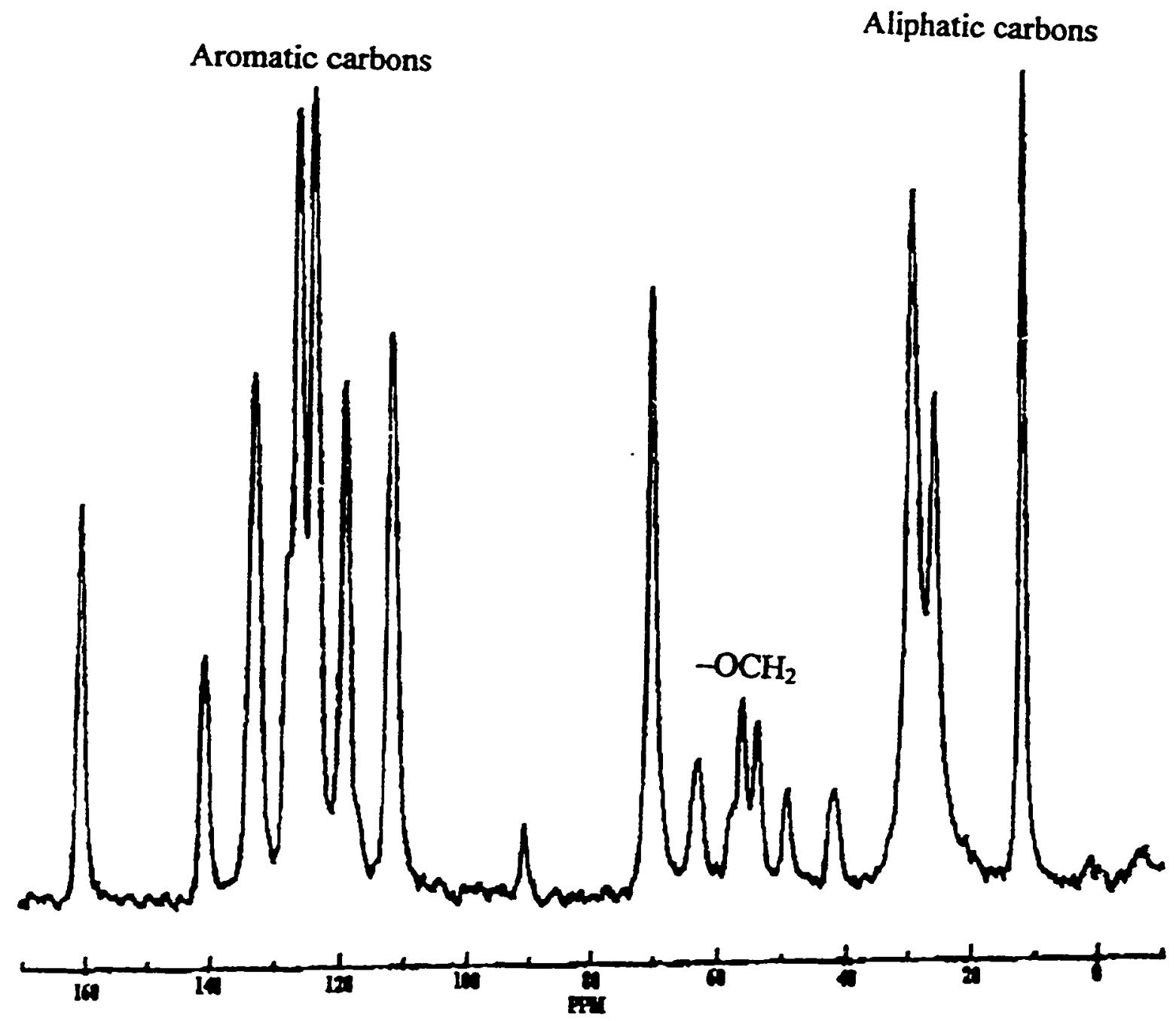

Figure 40. ${ }^{13} \mathrm{C}$ CP-MAS NMR spectrum of 4-cyano-4'-n-pentoxy -1,1'-biphenyl starting material 


\section{3a. ${ }^{13} \mathrm{C}$ CP-MAS NMR of starting material}

Figure 40 represents the ${ }^{13} \mathrm{C}$ CP-MAS NMR spectrum of 4-cyano-4'-n-pentoxy 1,1'-biphenyl starting material. In this spectrum the peaks in the range of $12.1 \mathrm{ppm}$ to $70.1 \mathrm{ppm}$ are due to the aliphatic carbons present. The peak at $70.1 \mathrm{ppm}$ is due to the $-\mathrm{OCH}_{2}$ group. The peak at $12.1 \mathrm{ppm}$ represents the methyl group of the liquid crystal material. The peaks in the range of $119.1 \mathrm{ppm}$ to $160.5 \mathrm{ppm}$ are from the aromatic carbons in the liquid crystalline material. The peak at $140.9 \mathrm{ppm}$ is due to the cyano group. The cross- polarization of this group is restricted due to the absence of hydrogen resulting in a smaller peak.

\section{3b. ${ }^{13} \mathrm{C}$ CP-MAS NMR of bonded phase}

The ${ }^{13} \mathrm{C}$ CP-MAS NMR spectrum of 4-cyano-4'-n-pentoxy $-1,1^{\prime}$-biphenyl bonded to Kromasil hydride is shown in Figure 41 . This spectrum is very different compared to that of the starting material. This spectrum shows very few peaks and most of the peaks due to the alkyl groups are not seen at all. There are two peaks (139 ppm and $127 \mathrm{ppm}$ ) in the aromatic region and only one peak in the aliphatic region. The reason for such an observation could be either a very high degree of association between the adjacent bonded moieties or very long relaxation period for the carbons in this region of bonded group. The peak at $65.2 \mathrm{ppm}$ is due to the $-\mathrm{OCH}_{2}$ group. This spectrum is very unusual because there are no peaks for the alkyl groups despite the presence of a long alkyl chain in the liquid crystal moiety. The presence of a peak at $65.2 \mathrm{ppm}$ due to $-\mathrm{OCH}_{2}$ group overlaps with the $-\mathrm{CH}_{2} \mathrm{~N}$ group. Therefore, the possibility of the peak due to bonding group cannot be neglected. Moreover, the peak at $65.2 \mathrm{ppm}$ due to $-\mathrm{OCH}_{2}$ and 
the peak at $127 \mathrm{ppm}$, indicating the presence of aromatic carbons, confirms the success of bonding.

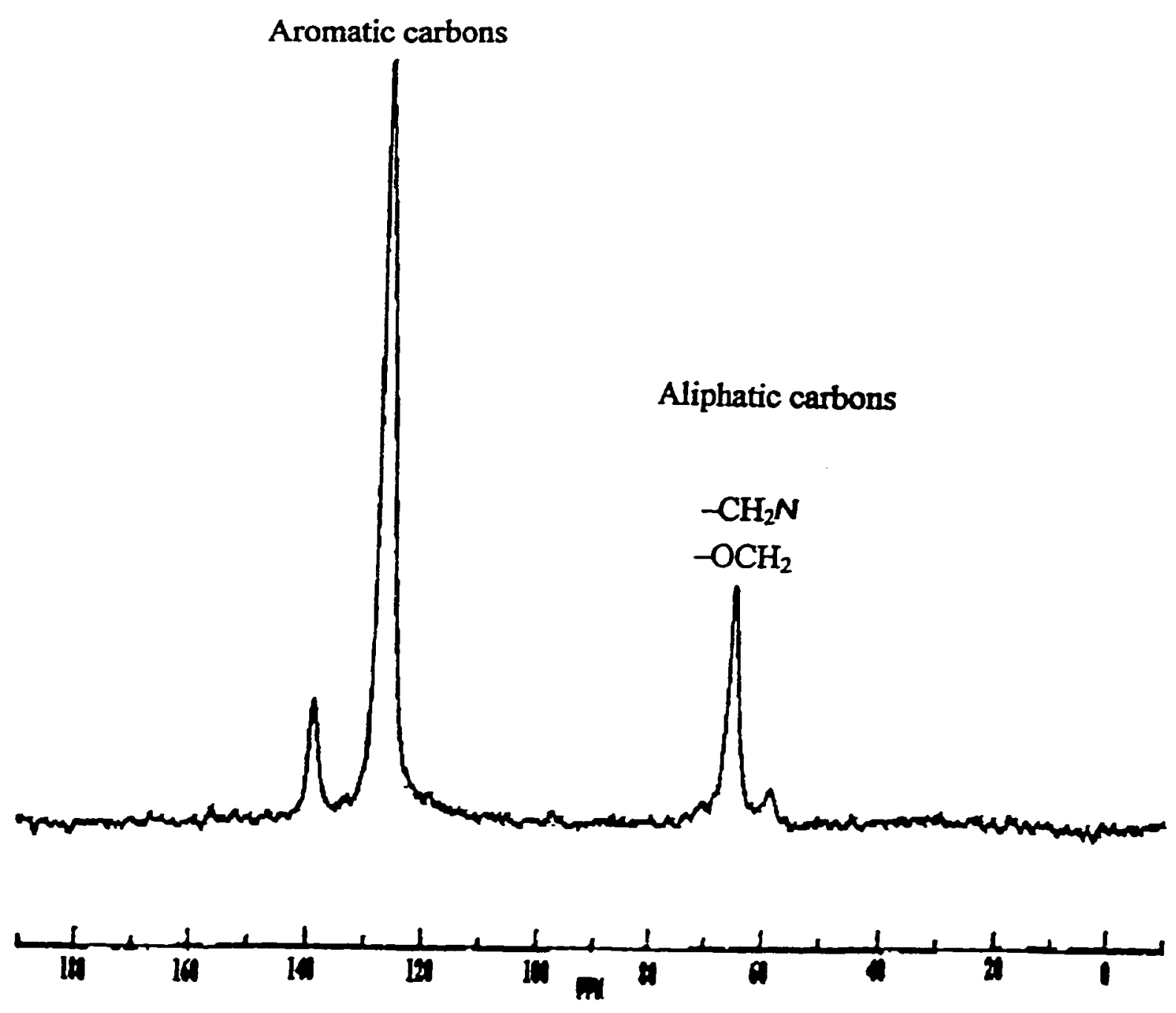

Figure 41. ${ }^{13} \mathrm{C}$ CP-MAS NMR spectrum of 4-cyano-4'-n-pentoxy -1,1'-biphenyl bonded to Kromasil hydride 


\section{G. Chromatographic Studies}

\section{4-cyano-4'- n- pentoxy-1,1'-biphenyl stationary phase}

\section{a. Separation of standard reference mixture (SRM 869)}

Polycyclic aromatic hydrocarbons (PAHs) have been already separated successfully using the cholestereyl 10-undecanoate bonded phase. Therefore, these compounds are excellent test solutes for separations using other liquid crystal stationary phases. Moreover, PAHs can be major pollutants in the atmosphere and possess mutagenic and carcinogenic properties [18]. One of the first samples tested on this column was a standard reference mixture, SRM 869. It is a three component mixture consisting of the polycyclic aromatic hydrocarbons benzo[a]pyrene (BaP), phenanthro[3,4-c]-phenanthrene (PhPh) and tertabenzonaphthalene (TBN). The separation of this mixture was carried out in the isocratic mode using methanol: water $(90: 10, v / v)$ as the mobile phase. The chromatogram of this mixture is shown in Figure 42. The elution order is benzo[a]pyrene (BaP) followed by phenanthro[3,4-c]phenanthrene $(\mathrm{PhPh})$ and tertabenzonaphthalene (TBN). The chromatographic results indicate that retention increases with the number of aromatic rings and also the planarity of the rings in the solute.

The capacity factor $k^{\prime},\left\{k^{\prime}=\left(t_{r}-t_{0}\right) / t_{0}\right\}$ for each of these compounds was determined from their retention times. The retention time of the solute is given by $t_{r}$ and $t_{0}$ is the retention time of an unretained compound. Further, the separation factor, $\alpha$, for pairs of these compounds were also calculated [26]. The separation factor is calculated by $\alpha=k_{2}^{\prime} / k_{1}^{\prime}$, where $k_{1}^{\prime}$ and $k_{2}^{\prime}$ are the capacity factors of the two compounds. The 


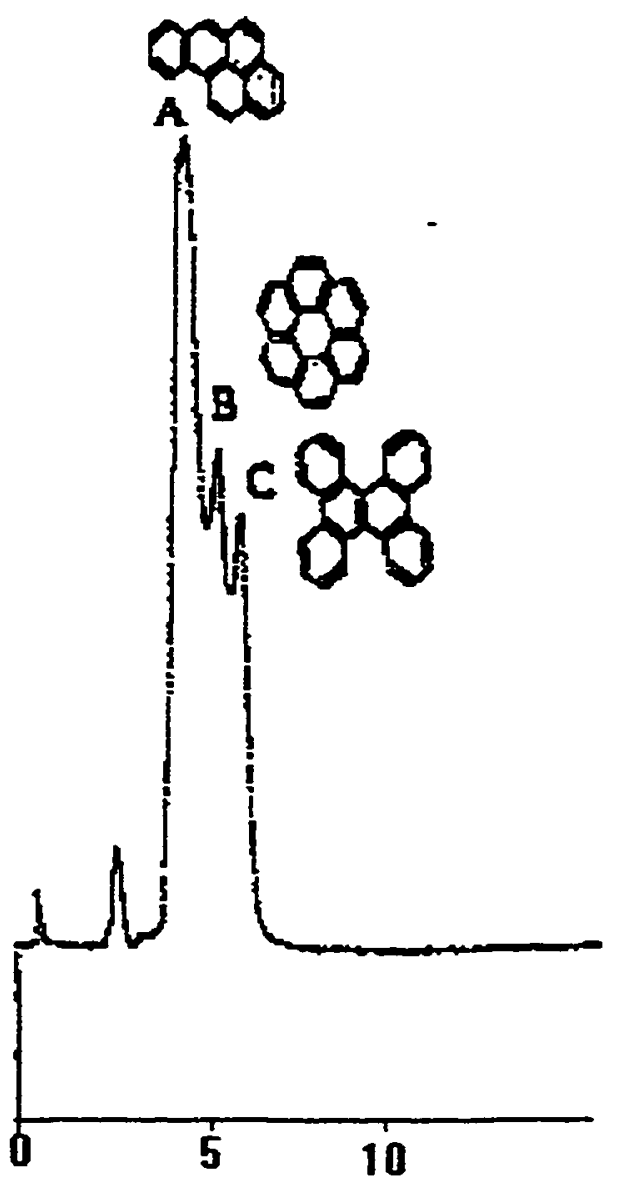

Figure 42. Reversed phase separation of SRM 869 mixture on a 4-cyano-4'-npentoxy-1,1'-biphenyl column; mobile phase : water : methanol, v/v (10: 90 ), at 0.5 $\mathrm{mL} / \mathbf{m i n}$; sample injection $20 \mu \mathrm{l}$; detection : $\mathrm{UV}$ at $254 \mathrm{~nm}$; peaks : $\mathrm{A}=$ Benzo[a]pyrene, $B=$ Phenanthro[3,4-c]phenanthrene and $C=$ Tetrabenzonaphthalene 
value of $\alpha$, for $\mathrm{PhPh}\left(\mathrm{k}_{2}{ }^{\prime}\right)$ and BaP $\left(\mathrm{k}_{1}{ }^{\prime}\right)$ was 1.42 and that for TBN $\left(\mathrm{k}_{3}{ }^{\prime}\right)$ and BaP $\left(k_{1}{ }^{\prime}\right)$ was equal to 1.32. These separation factor values are very close to that obtained for a highly loaded monomeric phase. An $\alpha$ value in the range of $1.5-2$ is typical of a octadecylsilica (ODS) monomeric phase [27]. Therefore this stationary phase can be classified as monomeric phases or more appropriately an intermediate phase.

\section{b. Separation of Perkin-Elmer universal mixtare}

The structures of the compounds present in the Perkin-Elmer test mixture are shown in Table 4. The separation was carried out in the isocratic mode using methanol and water $(20: 80, v / v)$ as the mobile phase. The chromatogram is presented in Figure 43. The elution order is anthracene $>t$ - butyl peroxide $>$ isopropyl benzene $>$ ethyl benzene $>$ toluene $>$ benzene. This is typical of a reverse phase mode [28]. The retention data suggests that for planar compounds, retention increases with the size and the length/breath ratios of the solute.

\section{c. Separation of benzodiazipines}

Benzodiazipines are another group of compounds that have been previously separated successfully using cholesteryl 10-undecanoate [17]. These compounds are widely used as psychotropic drugs because of their sedative or hypnotic effects. Since these drugs have high risk for abuse, any new and improved method for their analysis is very useful. The cholesteryl 10 - undecanoate phase was very selective for these closely related compounds because of its liquid crystalline property. Therefore, benzodiazpines were chosen for separation using the newly synthesized liquid crystal stationary phases in this study. 
Five of the most common benzodiazipines were used for this study. Their structures are shown in Table 5.

\section{Perkin-Elmer Mixture}

\begin{tabular}{|c|c|c|c|}
\hline Compound & Structure & Compound & $\begin{array}{c}\text { isopropyl- } \\
\text { benzene }\end{array}$ \\
benzene & Structure \\
ethylbenzene & benzene \\
anthracene
\end{tabular}

Table 4. Perkin-Elmer mixture (structures) 


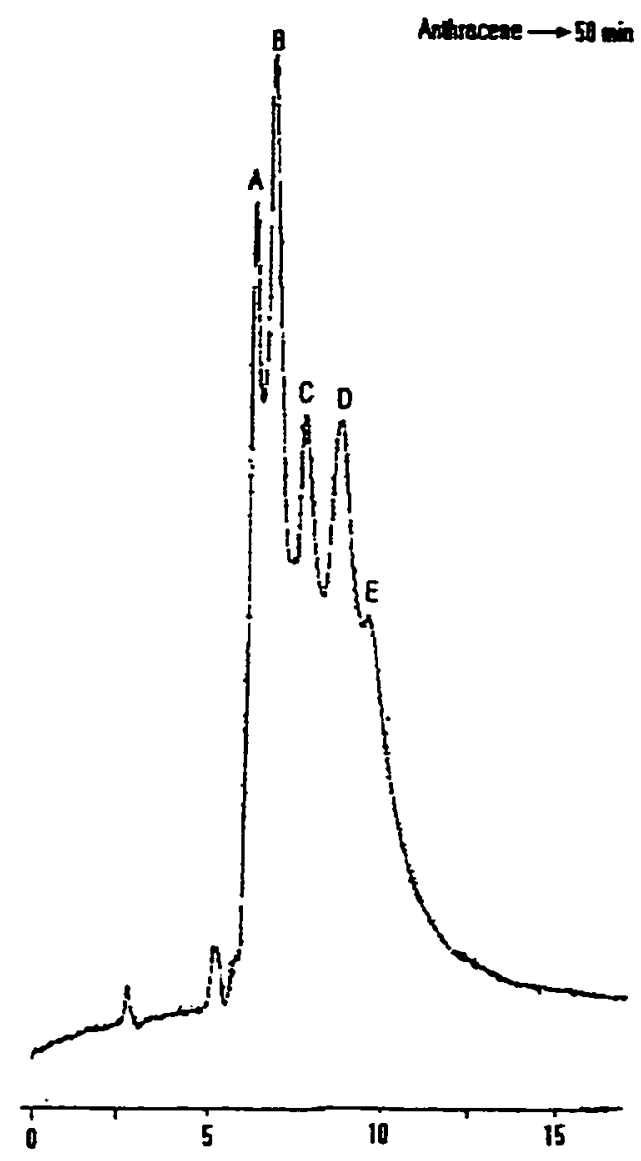

Figure 43. Reversed phase separation of PE universal test mixture on a 4-cyano-4'n-pentoxy-1,1'-biphenyl column; mobile phase : water : methanol, v/v (80:20), at $0.5 \mathrm{ml} / \mathrm{min}$; sample injection $5 \mu \mathrm{l}$; detection : $\mathrm{UV}$ at $254 \mathrm{~nm}$; peaks : $A=$ benzene, B $=$ toulene, $\mathrm{C}=$ ethylbenzene, $\mathrm{D}=$ isopropyl benzene, $\mathrm{E}:$ tertiary butyl benzene, anthracene was eluted at $\mathbf{5 0}$ min. 


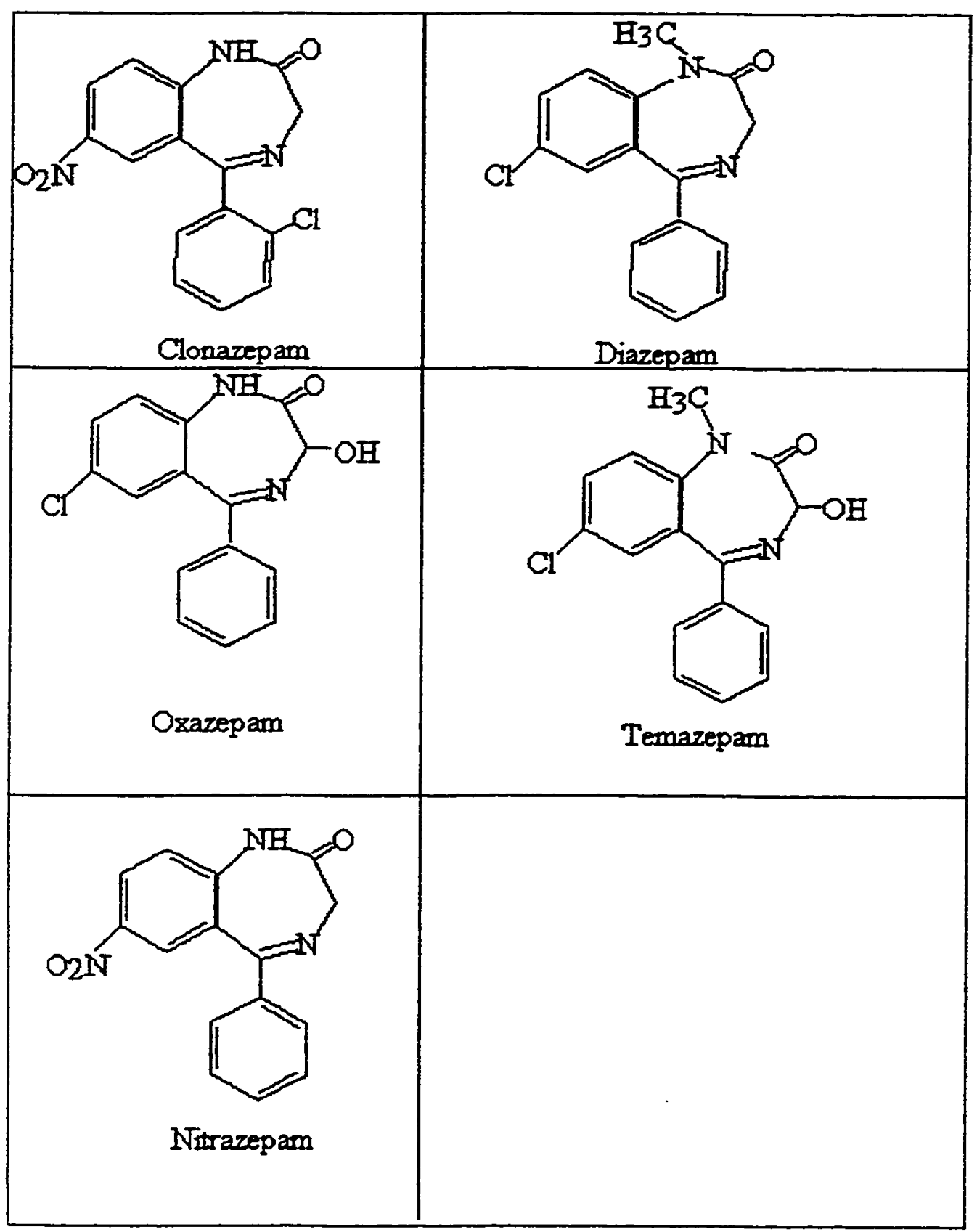

Table 5. Benzodiazipine structures 
Samples were extracted by dissolving the powdered tablets in methanol and then evaporated by heating. The concentrated mixture thus obtained was filtered to remove impurities. The separations of these compounds using two different mobile phase compositions are shown in Figure $\mathbf{4 4}$ and Figure 45.

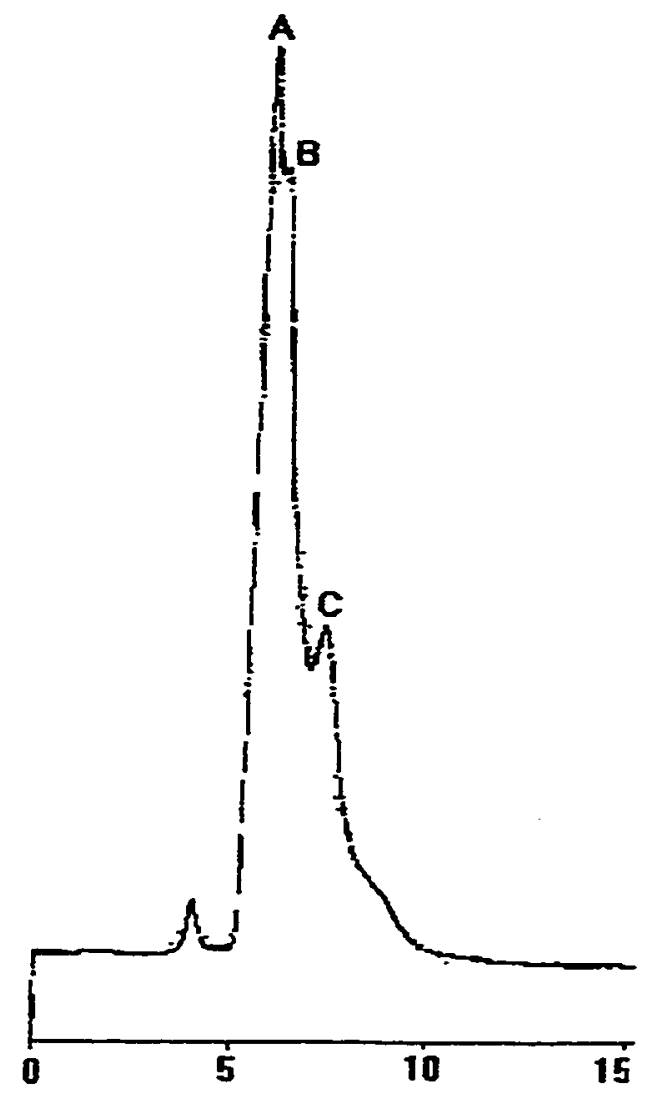

Figure 44. Reversed phase separation of benzodiazipines mixture on a 4-cyano-4'-npentoxy-1,1'-biphenyl column; mobile phase : water : methanol, v/v ( 65 :35), at 0.4 $\mathrm{mL} / \mathrm{min}$; sample injection $20 \mu \mathrm{l}$; detection : $\mathrm{UV}$ at $254 \mathrm{~nm}$; peaks : $A=$ Orazepam + Clonazepam , B $=$ Nitrazepam + Temazepam , C $=$ Diazepam. 


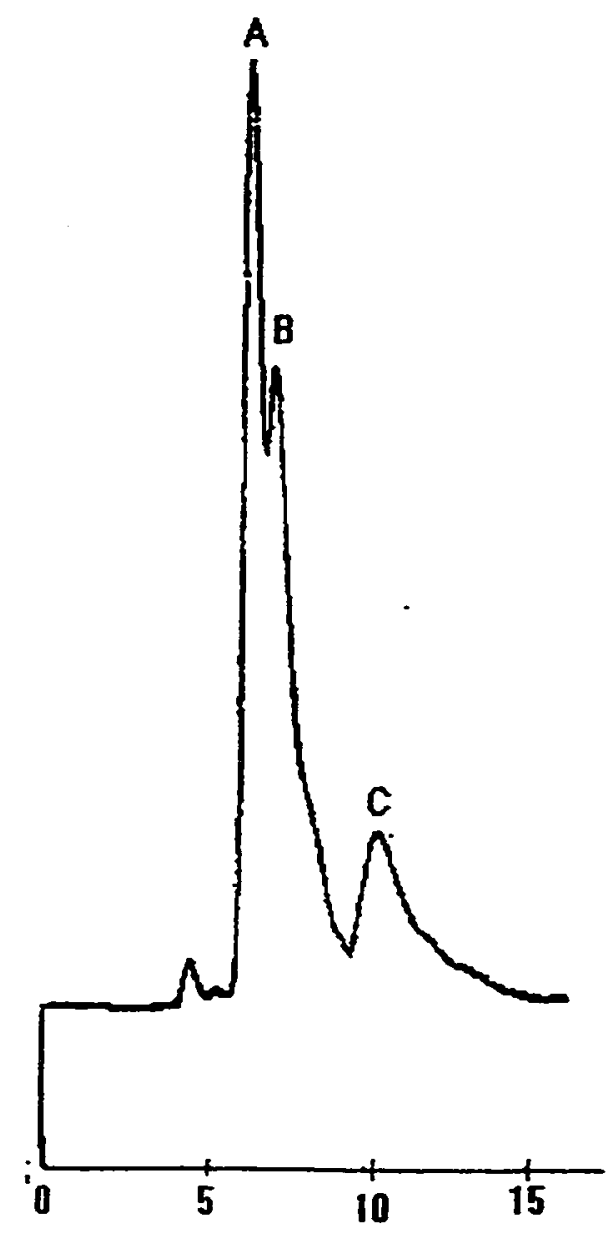

Figure 45. Reversed phase separation of benzodiazipines mixture on a 4-cyano-4'-npentoxy-1,1'-biphenyl column; mobile phase : water : methanol, v/v ( 75 :25 ), at 0.4 mV/min; sample injection $20 \mu$ l ; detection : UV at $254 \mathrm{~nm}$; peaks : A = Oxazepam + Clonazepam , B = Nitrazepam + Temazepam , $C=$ Diazepam. 
Tables 6 and 7 show the capacity factors of these compounds.

\begin{tabular}{|c|c|}
\hline Compound & $\mathbf{k}^{\prime}$ \\
\hline Oxazepam & 0.24 \\
\hline Clonazepam & 0.32 \\
\hline Nitrazepam & 0.35 \\
\hline Temazepam & 0.39 \\
\hline Diazepam & 0.82 \\
\hline
\end{tabular}

Table 6. Capacity factors of benzodiazipines on 4-cyano-4'-n - pentoxy-1,1'biphenyl column

Column: 4-Cyano-4'-N-Pentoxy-1,1'-Biphenyl

Mobile Phase: Water. Methanol (65:35, v/v)

Flow rate: $0.4 \mathrm{~mL} / \mathrm{min}$

Pressure: 82 bar

Sample: Benzodiazepine mixture

\begin{tabular}{|c|c|}
\hline Compound & $\mathbf{k}^{\prime}$ \\
\hline Oxazepam & 0.18 \\
\hline Clonazepam & 0.19 \\
\hline Nitrazepam & 0.21 \\
\hline Temazepam & 0.28 \\
\hline Diazepam & 0.57 \\
\hline
\end{tabular}

Table 7. Capacity factor of benzodiazipine mixture using 4-cyano-4'-n-pentoxy-1, 1'biphenyl column

Column: 4-Cyano-4'-N-Pentoxy-1,1'-Biphenyl

Mobile Phase: Water: Methanol (75: 25, v/v)

Flow rate: $0.4 \mathrm{~mL} / \mathrm{min}$

Pressure: 86 bar

Sample: Benzodiazepine mixture 
It was observed that the separation was better at a higher percentage of water. Also, the elution order of the benzodiazipines is slightly different than the one obtained for the 4 cyano-4'-n-pentyl-1,1'-biphenyl bonded phase. The polar nitro group of nitrazepam interacts less with the stationary phase and therefore should be the least retained. However, clonazepam and oxazepam are retained less than nitrazepam. Temazepam and Diazepam are retained longer on the column based on the hydrophobic interactions with the long alkyl chain of the stationary phase.

\section{4-Cyano-4'-n-pentyl-1,1'-biphenyl stationary phase}

\section{a. Separation of standard reference mixture (SRM 869)}

The three component mixture was injected into the 4-cyano-4'-n-pentyl-1, 1'biphenyl column using methanol: water $(70: 30, v / v)$ as the mobile phase. The separation was carried out at a flow rate of $0.5 \mathrm{ml} / \mathrm{min}$ in an isocratic mode. The chromatogram is shown in Figure 46.

Figure 47 also shows the separation of SRM 869 mixture under isocratic conditions. This separation was carried out using methanol: water $(80: 20, \mathrm{v} / \mathrm{v})$ as mobile phase at a flow rate of $0.5 \mathrm{ml} / \mathrm{min}$. The separation factor $\alpha$, for TBN and $\mathrm{BaP}$ is equal to 1.89 , which is typical of a highly loaded monomeric phase [27].

\section{b. Separation of Perkin-Elmer test mixture}

The separation of the Perkin-Elmer mixture is shown in Figure 48. The separation was carried out under different isocratic conditions. It was observed that the separation was not as good compared to the other liquid crystal column prepared for this study. The best separation was obtained using $20 \%$ methanol in water as the mobile phase. At a higher percentage of methanol there was only one peak. Benzene and toluene are eluted 
as peak $A$ and ethyl benzene, isopropyl benzene, $t$ - butyl benzene and anthracene are eluting as peak $B$.

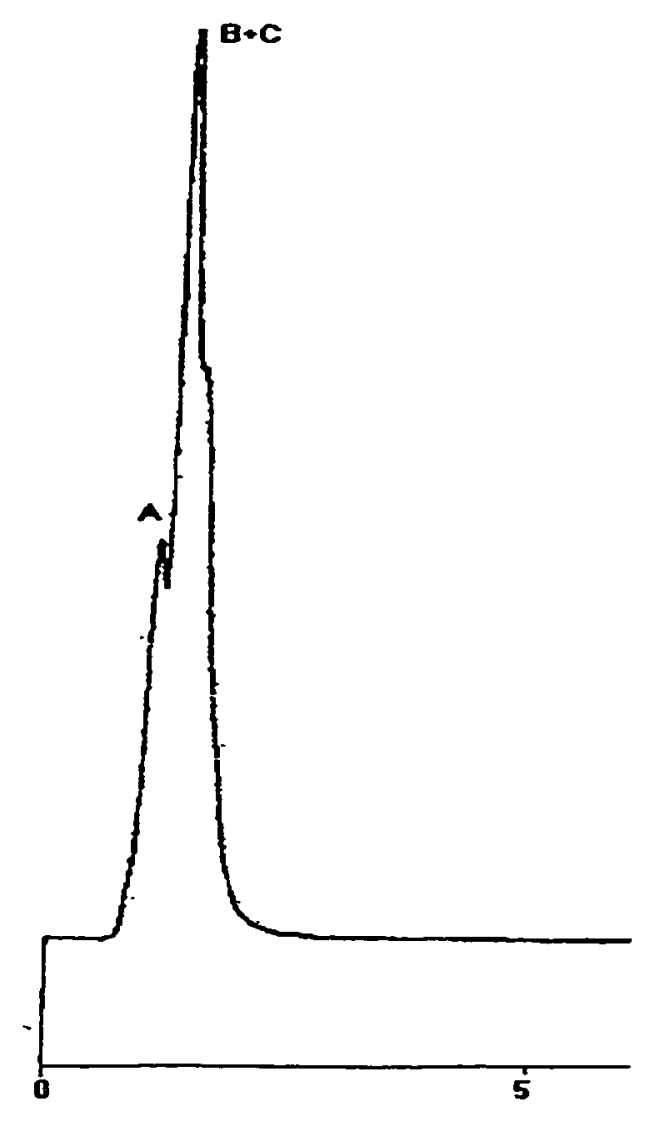

Figure 46. Reversed phase separation of NIST mixture (SRM 869) on a 4-cyano-4'n-pentyl-1, 1'-biphenyl column; mobile phase - water: methanol, v/v (30:70), at 0.5 $\mathrm{mL} / \mathrm{min}$; sample injection $20 \mu \mathrm{l}$; detection : $\mathrm{UV}$ at $254 \mathrm{~nm}$; peaks : $A=$ Benzo[a]pyrene, $B+C=$ Phenanthro[3,4-c]phenanthrene and Tetrabenzonaphthalene. 


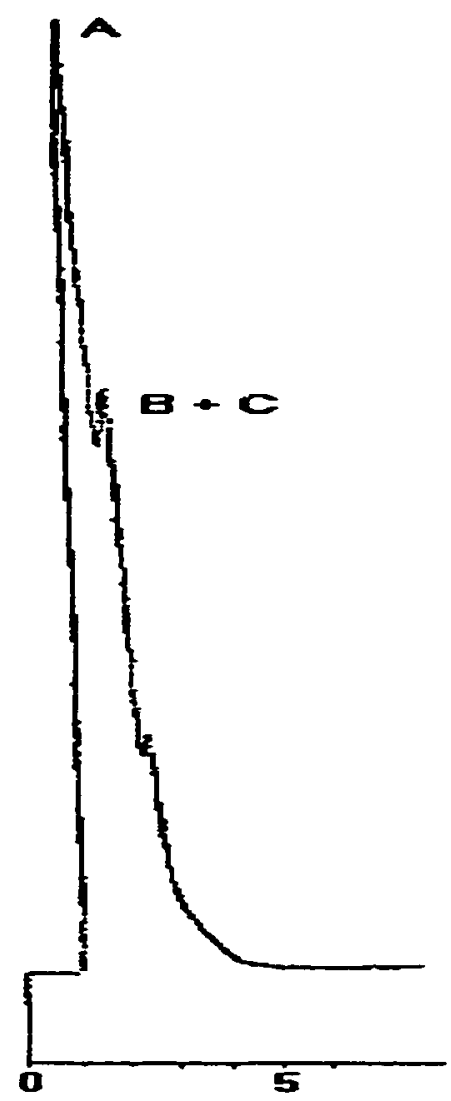

Figure 47. Reversed phase separation of NIST mixture (SRM 869) on a 4-cyano-4'n-pentyl-1,1'-biphenyl column; mobile phase : water : methanol, v/v ( $20: 80)$, at 0.5 $\mathrm{mL} / \mathrm{min}$; sample injection $20 \mu \mathrm{l}$; detection : UV at $254 \mathrm{~nm}$; peaks : $A=$ Benzo[a]pyrene, $B+C=$ Phenanthro[3,4-c]phenanthrene and Tetrabenzonaphthalene 


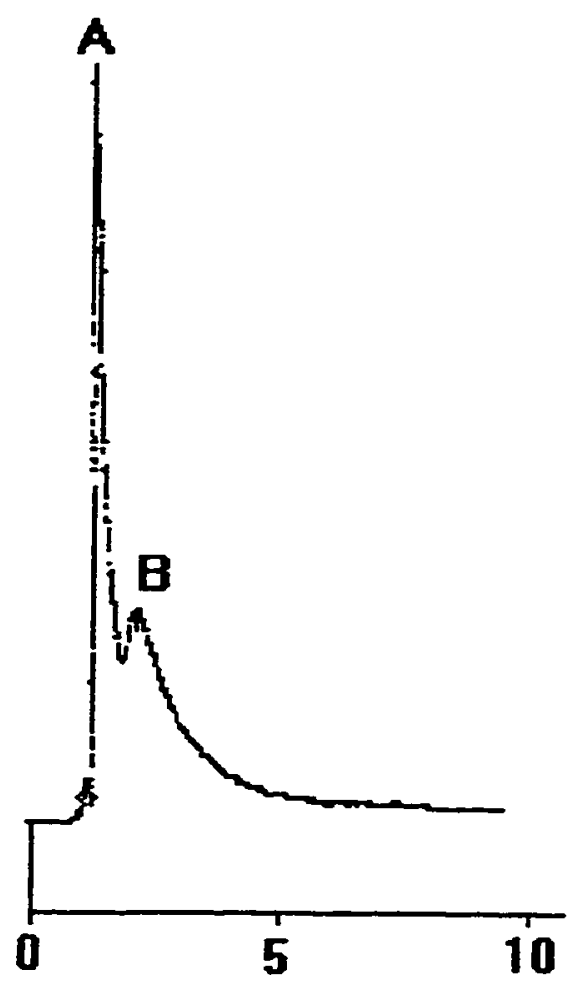

Figure 48. Reversed phase separation of $P E$ universal test mixture on a 4-cyano-4'n-pentyl-1,1'-biphenyl column; mobile phase : water : methanol, v/v (80:20), at 0.5 $\mathrm{ml} / \mathrm{min}$; sample injection $20 \mu$; detection : UV at $254 \mathrm{~nm}$; peaks : peak A:benzene $\&$ toluene; peak B: ethyl benzene, isopropyl benzene, $t$ - butyl benzene and anthracene

c. Separation of benzodiazipines

Figure 49 and Figure 50 show the separation of benzodiazipines on the 4 -cyano$4^{\prime}-n$ - pentyl $-1,1^{\prime}$ - biphenyl column at two different mobile phase conditions. 


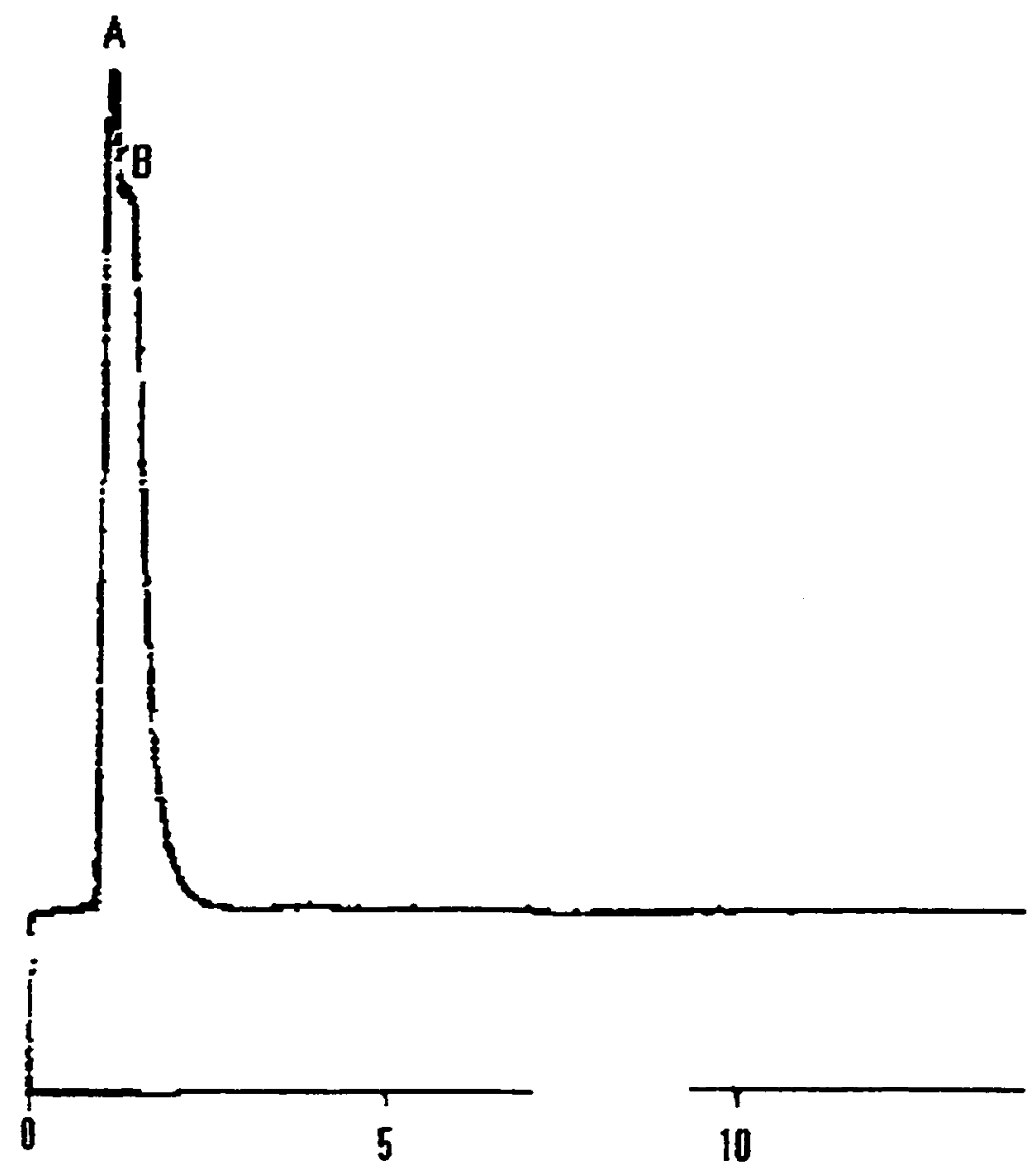

Figure 49. Reversed phase separation of benzodiazipines mixture on a 4-cyano-4'-npentyl-1,1'-biphenyl column; mobile phase : water : methanol, v/v (65: 35 ), at 0.5 mL/min; sample injection $20 \mu \mathrm{l}$; detection : UV at $254 \mathrm{~nm}$; peaks : A=Nitrazepam +Clonazepam + Oxazepam, B = Temazepam + Diazepam 


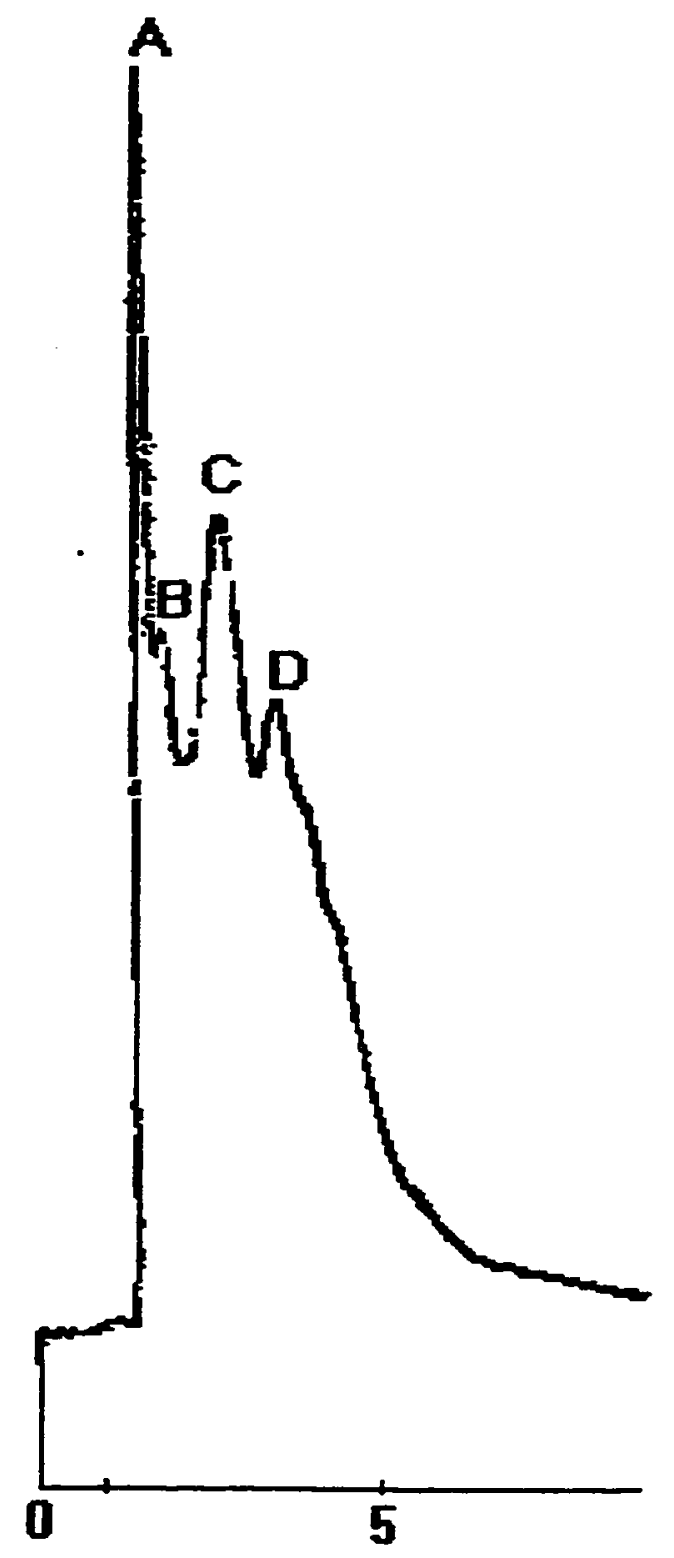

Figure 50. Reversed phase separation of benzodiazipines mixture on a 4-cyano-4'-npentyl-1,1'-biphenyl column; mobile phase : water : methanol, v/v $(80: 20)$, at 0.5 $\mathrm{ml} / \mathrm{min}$; sample injection $20 \mu \mathrm{l}$; detection : $\mathrm{UV}$ at $254 \mathrm{~nm}$; peaks : $A=$ Solvent peak, B $=$ Nitrazepam + Clonazepam + Oxazepam, C $=$ Temazepam, $D=$ Diazepam 
The separation in Figure 49 was carried out with methanol: water $(35: 65, \mathrm{v} / \mathrm{v})$. The capacity factor data for this separation is shown in Table 8. The separation of benzodiazipines using methanol: water $(20: 80, \mathrm{v} / \mathrm{v})$ as the mobile phase is shown in Figure 50. The separation was carried out at a flow rate of $0.5 \mathrm{~mL} / \mathrm{min}$. The capacity factor data is presented in the Table 9.

\begin{tabular}{|c|c|}
\hline Compound & $\mathbf{k}^{\prime}$ \\
\hline Nitrazepam & 0.58 \\
\hline Clonazepam & 0.69 \\
\hline Oxazepam & 0.78 \\
\hline Temazepam & 1.13 \\
\hline Diazepam & 1.60 \\
\hline
\end{tabular}

Table 8. Capacity factors for benzodiazipine mixture using 4-cyano-4'-n-pentyl-1,1'biphenyl column

Column: 4-Cyano-4'-N-Pentyl-1,1'-Biphenyl

Mobile Phase: Water: Methanol (65\%:35\%)

Flow rate: $0.5 \mathrm{~mL} / \mathrm{min}$

Pressure: 60 bar

Sample: Benzodiazepines mixture

For both separations the elution order was essentially same except that at a higher percentage of water temazepam and diazepam were well separated. The retention data shows that presence of a methyl group at the $\mathrm{N}-1$ position in both temazepam and diazepam increases the retention time. This could be due to the hydrophobic interactions between these compounds and the long alkyl chain of the stationary phase. Temazepam differs from diazepam in having an additional hydroxyl group at position 3 . This makes it 
more polar compared to diazepam resulting in a decreased retention time. Nitrazepam and clonazepam both have a polar nitro group at position 8 . However, clonazepam also has an additional chlorine. Oxazepam contains a hydroxyl group at position 3. It also contains chlorine instead of a nitro group. Nitrazepam, oxazepam and clonazepam are eluted quickly as the polar $\mathrm{NO}_{2}$ and $\mathrm{OH}$ substituents interact less with the stationary phase and therefore are less retained.

\begin{tabular}{|c|c|}
\hline Compound & $\mathbf{k}^{\prime}$ \\
\hline Nitrazepam & 0.61 \\
\hline Clonazepam & 0.65 \\
\hline Oxazepam & 0.79 \\
\hline Temazepam & 1.0 \\
\hline Diazepam & 2.54 \\
\hline
\end{tabular}

Table 9. Capacity factors for benzodiazipine mixture using 4-cyano-4'-n-pentyl-1,1'biphenyl column

Column: 4-Cyano-4'-N-Pentyl-1,1'-Biphenyl

Mobile Phase: Water : Methanol (80\%:20\%)

Flow rate: $0.5 \mathrm{~mL} / \mathrm{min}$

Pressure: 63 bar

Sample: Benzodiazepines mixture

As described in the Introduction section, the liquid crystalline stationary phases show selectivity of the compounds based on their molecular recognition capabilities. The variations in the retention behavior between the two phases could be attributed to the differences in their bonded structure. The NMR spectrum of 4-cyano-4'-n-pentoxy-1,1'biphenyl bonded phase (Figure 40) indicates that there is a very high degree of 
association between the neighboring bonded groups. This could probably lead to reduced interaction of the solutes in the stationary phase because of the compactness of the material. Therefore the differences in the bonded phase structures are probably responsible for the variation in the elution order. 


\section{CHAPTER IV - CONCLUSTON}

Two liquid crystal stationary phases have been synthesized by TES silanization, followed by free radical initiation using tertiary butyl peroxide. These liquid crystal stationary phases were characterized by diffuse reflectance infrared Fourier transform spectroscopy, cross-polarization magic-angle spinning nuclear magnetic resonance spectroscopy and HPLC. This study was targeted at developing a novel bonded phase using liquid crystal compounds with a terminal cyano group. The main goal was to bond the liquid crystal compound to silica hydride through the cyano group for use as a stationary phase in HPLC. This type of bonding was achieved by using free radical initiation. The yield of the bonded phase could be improved by further optimizing the reaction conditions such using higher percentage of catalyst or increasing the reaction time.

Elemental analysis showed a surface coverage of $0.84 \mu \mathrm{mol} / \mathrm{m}^{2}$ and $0.88 \mu \mathrm{mol} / \mathrm{m}^{2}$ for 4-cyano-4'-n-pentoxy-1,1'-biphenyl and 4-cyano-4'-n-pentyl-1, $1^{\prime}$-biphenyl columns respectively. The most interesting result was the NMR spectrum for 4-cyano-4'-n-pentoxy $1,1^{\prime}$-biphenyl bonded group. This spectrum showed only one peak for the aliphatic portion of the bonded moiety despite the presence of a long alkyl chain. The high degree of association between the bonded moieties could in part account for this observation. Another reason could be the very long relaxation time of these carbons due to their rigid structure. The chromatographic data also suggested some major differences in the retention mechanism of the two liquid crystal stationary phases. Nitrazepam, one of the benzodiazipoines was retained a little longer on the 4 - cyano $-4^{\prime}-n$-pentoxy-1,1'- 
biphenyl column as compared to the $4-$ cyano $-4^{\prime}-n-p e n t y l-1,1^{\prime}-$ biphenyl column. One of the possible explanations for these differences is based on the differences in their bonded structures as indicated by the NMR spectroscopy. However, the exact explanation for this behavior is not completely understood. Future work is focused on investigating the selectivity of these columns as a function of temperature. Also, an extension of this study would include using these stationary phases for the separation of fat-soluble vitamins and proteins. 


\section{CHAPTER V - REFERENCES}

1. Nawrocki J. J. Chromatogr A. 1997, 779, 29-71.

2. Unger, K.K. Journal of chromatography library, Elsevier Scientific, 1979, 16, Ch-2,3.

3. Pesek, J.J.;Matyska, M.T.; Sandoval, J.E.; Williamsen, E.J. J.Liq.Chrom.\& Rel.Technol. 1996, 19, 2843-2865.

4. Pesek, J.J.;Matyska, M.T. Interface Science, 1997, 5, 103 - 117.

5. Chu, C.J.; Jonsson,E.J.; Anvinen, M.; Pesek, J.J.; Sandoval, J.E. Anal.Chem., 1993, $65,808-816$.

6. Marciniec, B.; Gulinski, J.; Urbaniak, W.; Kornetka, W. Comprehensive Handbook on Hydrosilylation, Pegamon Press, Oxford, 1992, Ch. 2.

7. Speier, J.L. Organomet. Chem., 1979, 17, 401.

8. Pesek, J.J.; Matyska, M.T.; Hemphala, H.; Christensen, P.J. Liq.Chromatogr., 1995, $18,2507-2526$

9. Pesek, J.J.;Matyska, M.T.; Williamsen, E.J.; Evanchic, M.; Hazari, V.; Konjuh, K.; Takhar, S.; Tranchina, R. J. Chromatogr A. 1997, 786, 219-228.

10. Lindsay, S.; High Performance Liquid Chromatography, 2nd ed.; John Wiley and Sons: Chichester, England, 1992, Ch. 1-3.

11. Hamilton, R.J.; Sewell, P.A. Introduction to High Performance Liquid Chromatography, $2^{\text {nd }}$ edition, Chapman and Hall: New York, 1982, Ch-1,3

12. Chandrasekhar, S. Liquid Crystals, 2nd ed.; Cambridge University Press: Cambridge 1992, Ch-1.

13. Shah, S. MS thesis, San Jose State University, 1991.

14. Dewar, M.J.S.; Schroeder, J.P. J. Am. Chem. Soc., 1964, 86, 5235-5239. 
15. Wise, S.A.; Sander, L.C. J. High Resolut. Chromatogr. Chromatogr. Commum., 1985, 8, 248.

16. Pesek, J.J.; Williamsen, E. J. Trends in Analytical Chem. 1992, 11(7), 259-264.

17. Catabay, A.; Taniguchi, M; Jinno, K.; Pesek, J.J.; Williamsen, E. J. J.Chromatograpic Science. 1998, 36, 111- 118.

18. Catabay, A.; Okumura, C.; Jinno, K.; Pesek, J.J.; Williamsen, E.;Fetzer, J.C.; Biggs, W.R. Chromatographia. 1998, 47, 13-20.

19. Jinno, K.; Saito, Y.; Malhan n'ee Chopra, R., Pesek, J.J.; Fetzer, J.C.; Biggs, W.B. J. Chromatogr, 1991, 557, $459-468$.

20. Catabay, A.; Saito, Y.; Okumura, C.; Jinno, K.; Pesek, J.J.; Williamsen, E. J. Micro.Sep. 1997, 9, 81-85.

21. Shen, Y.; Bradshaw, J.S.; Lee, M.L. Chromatographia, 1996, 43, 53-58.

22. Jinno, K.; Mae, H.; Saito, Y. J. Microcol. Sep. 1991, 3, 417-422.

23. Pesek, J.J.; Matyska, M.T. Spectroscopic Characterization of Chemically Modified Surfaces. Dept. of Chem., San Jose State University, CA, p 1-10.

24. Brendsen, G.E.; Degalen, L. J. Liq. Chromatogr., 1978, I, 561-568.

25. Pesek, J.J.; Matyska, M.T.; Williamsen, E.J.; Tam, R. Chromatographia, 1995, 4I, $301-310$.

26. Sander, L.C.; Wise, S.A. Anal.Chem., 1987, 59, 2309-2315.

27. Sander, L.C.; Wise, S.A. J. Chromatogr. A, 1993, 656, 335-351.

28. Matysks, M. T.; Golkiewicz, W.; J.Liq.Chromatogr., 1992, 598, 59. 